\title{
Decorrelation of MIMO Channels with Distributed Base Station Antennas
}

by

\author{
Nadem Hussein Dawod
}

A thesis submitted to the
Faculty of Graduate Studies and Research
in partial fulfillment of the requirements for the degree of

Doctor of Philosophy

in

Electrical Engineering

\begin{abstract}
Ottawa-Carleton Institute for Electrical and Computer Engineering Department of Systems and Computer Engineering

Faculty of Engineering

Carleton University

Ottawa, Ontario, Canada
\end{abstract}

(C) Nadem Dawod, 2006 


$\begin{array}{ll}\begin{array}{l}\text { Library and } \\ \text { Archives Canada }\end{array} & \begin{array}{l}\text { Bibliothèque et } \\ \text { Archives Canada }\end{array} \\ \begin{array}{l}\text { Published Heritage } \\ \text { Branch }\end{array} & \begin{array}{l}\text { Direction du } \\ \text { Patrimoine de l'édition }\end{array} \\ \begin{array}{l}\text { 395 Wellington Street } \\ \text { Ottawa ON K1A ON4 }\end{array} & \begin{array}{l}\text { 395, rue Wellington } \\ \text { Ottawa ON K1A ON4 } \\ \text { Canada }\end{array}\end{array}$

Your file Votre référence ISBN: 978-0-494-18212-3 Our file Notre référence ISBN: 978-0-494-18212-3

NOTICE:

The author has granted a nonexclusive license allowing Library and Archives Canada to reproduce, publish, archive, preserve, conserve, communicate to the public by telecommunication or on the Internet, loan, distribute and sell theses worldwide, for commercial or noncommercial purposes, in microform, paper, electronic and/or any other formats.

The author retains copyright ownership and moral rights in this thesis. Neither the thesis nor substantial extracts from it may be printed or otherwise reproduced without the author's permission.
AVIS:

L'auteur a accordé une licence non exclusive permettant à la Bibliothèque et Archives Canada de reproduire, publier, archiver, sauvegarder, conserver, transmettre au public par télécommunication ou par l'Internet, prêter, distribuer et vendre des thèses partout dans le monde, à des fins commerciales ou autres, sur support microforme, papier, électronique et/ou autres formats.

L'auteur conserve la propriété du droit d'auteur et des droits moraux qui protège cette thèse. $\mathrm{Ni}$ la thèse ni des extraits substantiels de celle-ci ne doivent être imprimés ou autrement reproduits sans son autorisation.
In compliance with the Canadian

Privacy Act some supporting forms may have been removed from this thesis.

While these forms may be included in the document page count, their removal does not represent any loss of content from the thesis.
Conformément à la loi canadienne sur la protection de la vie privée, quelques formulaires secondaires ont été enlevés de cette thèse.

Bien que ces formulaires aient inclus dans la pagination, il n'y aura aucun contenu manquant.

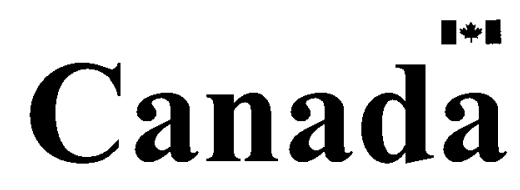




\begin{abstract}
Characterized by multiple antennas at both ends of a wireless link, multiple-input multipleoutput (MIMO) communication systems potentially offer a tremendous increase in data rate compared to single-antenna systems, without the need for additional power or bandwidth. This is possible because several spatial subchannels are opened between the transmitter and the receiver, through which several data symbols can be spatially multiplexed. These spatial subchannels can be used to simultaneously serve either multiple users in a space division multiple access (SDMA) system or a single user in a time division multiple access system. Ideally, the antennas are placed so that the corresponding MIMO channel matrix has uncorrelated elements. Unfortunately, because of practical physical constraints on the positioning of antennas, correlations within the channel matrix are unavoidable. These correlations diminish the degree of independence between the subchannels, which in turn significantly reduce channel capacity as compared to the ideal, uncorrelated, case.

To help reduce the channel matrix correlations, in this thesis we recommend distributing the basestation antennas as small arrays of antennas at several different sites around the microcell, instead of placing them all at a single site. We show this to be a very effective strategy for recovering some of the throughput loss compared to the uncorrelated case.

To further reduce the degradation due to correlations, we propose novel correlation reduction algorithms (decorrelation algorithms) based on antenna and user selection. In addition to reducing system complexity (since only a limited number of antennas may be active at any given time), these algorithms aim at improving the MIMO channel ergodic capacity by reducing channel matrix correlations while enhancing the spatial separability of the mobile users in a multi-user environment.

Performance evaluation results, obtained through computer simulation, confirm the advantages of distributing the basestation antennas, and demonstrate the effectiveness of the decorrelation algorithms. We consider a frequency selective Ricean fading MIMO channel with lognormal shadowing and correlated channel matrix entries (according to the angles of departure/arrival, angular spectrum and angular spread at each array) and with different pathlosses to each array site. We assume perfect channel knowledge at both the transmitter and receiver, and orthogonal frequency division multiplexing (OFDM) is used for transmission.
\end{abstract}




\section{Acknowledgements}

I would like to express my sincerest gratitude to my thesis supervisors, Professor Roshdy Hafez and Professor Ian Marsland, for their insightful guidance, support, generosity and constant encouragement throughout all these years.

I am very thankful to Prof. Hafez for his research support, sharp feedback and for always believing in my capabilities especially when I was lost for a while. He inspired me to think critically, positively and confidently and build physical intuition on research problems. He has been a good mentor.

I am extremely grateful to Prof. Marsland for his guidance and streamlining my mathematical thinking and for his precise and in-depth feedback on research and paper manuscripts that helped me tremendously. I would like to thank him for his time for reading, correcting and commenting this thesis.

I also thank my friends and colleagues at the systems and computer engineering department for help, support and for making the time spent for studies so enjoyable.

I would like to thank my parents, especially my father who died just one year after my departure to Canada. He encouraged me to choose Canada for the Ph.D study. He made tremendous sacrifices to ensure that I had a good education.

Finally, I would like to show my sincerest thanks to my family for their patience, help and encouragement. They have helped me in every possible way to pursue and achieve my goals. They took care of all little things, and made sure that I had the time and resources to work hard with an open mind. 


\section{Table of Contents}

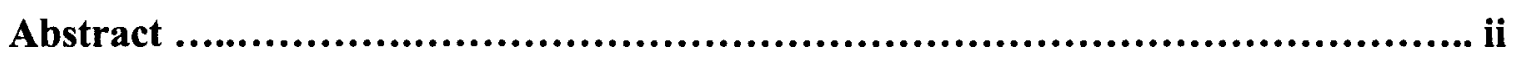

Acknowledgements................................................................................... iii

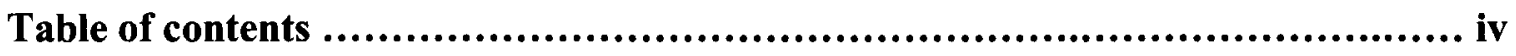

Abbreviations and Acronyms........................................................vii

Notations and symbols.......................................................... ix

List of tables..........................................................................xi

List of figures...................................................................... xii

1 Introduction and Motivation .............................................................. 1

1.1 Wireless Communication Systems...............................................................................1

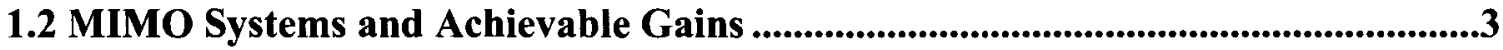

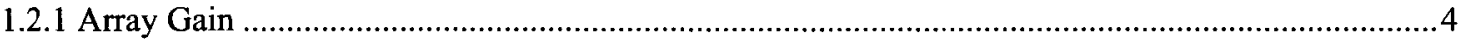

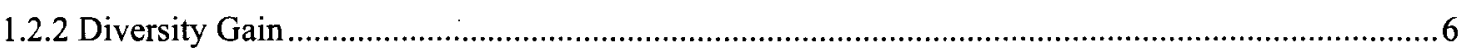

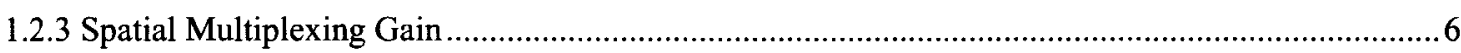

1.3 MIMO-OFDM Wireless Systems ...................................................................11

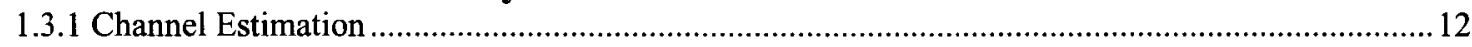

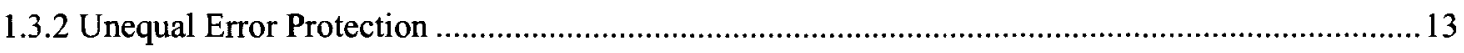

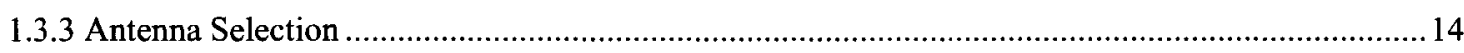

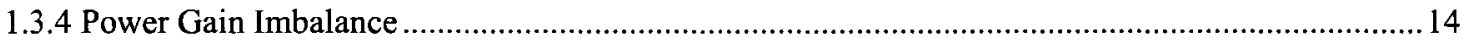

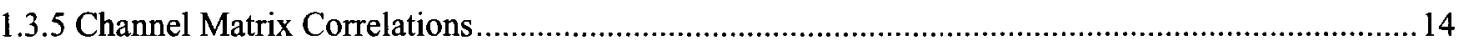

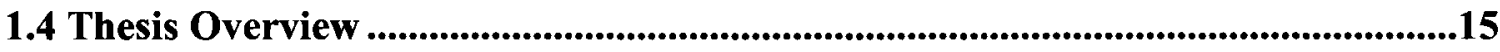

2 A MIMO Channel Model with Distributed Basestation Antennas ............. 21

2.1 Signal Distortion in Wireless Channels...................................................................21

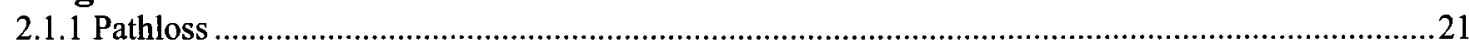

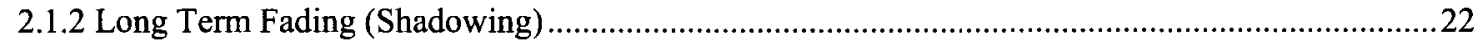

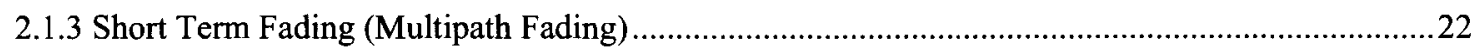


2.2 Physical Modeling of MIMO Channels.........................................................................24

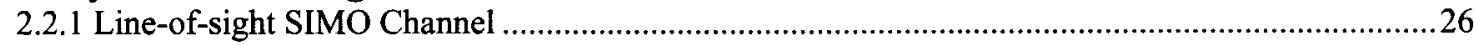

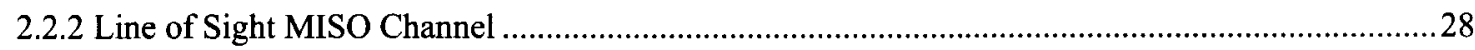

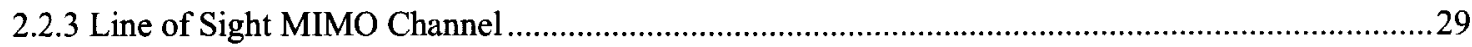

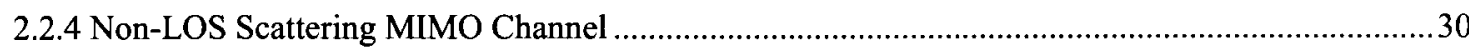

2.3 A MIMO Channel Model with Distributed Basestation Antennas ........................33

2.4 Space-Time OFDM ........................................................................................................................36

3 Capacity of Space-Time Channels ............................................................. 41

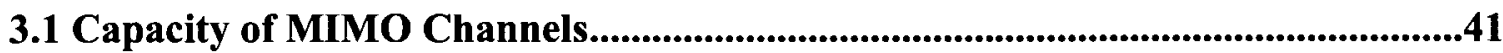

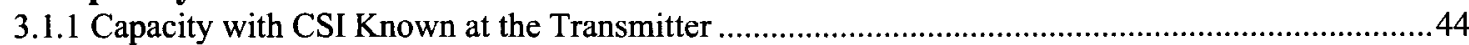

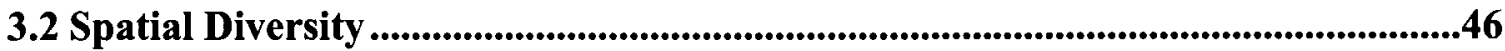

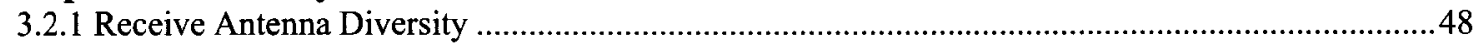

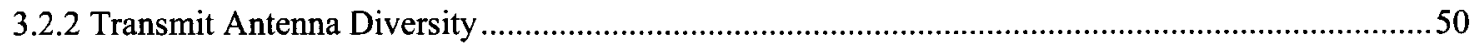

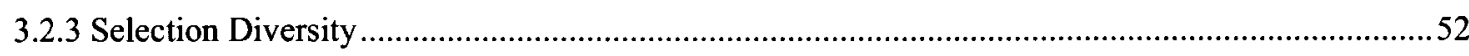

3.3 Impact of Channel Parameters on the Capacity ................................................57

3.3.1 Effect of Line Of Sight (LOS) on the MIMO channel capacity ................................................57

3.3.2 Effect of Correlation on the MIMO Channel Capacity ....................................................................57

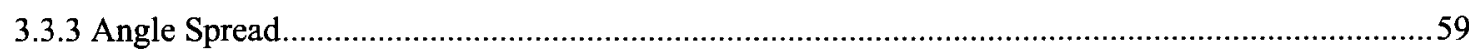

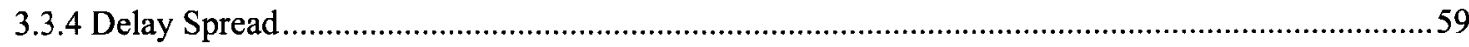

4 Selection Diversity in a MIMO Channel with Distributed Antennas ..........61

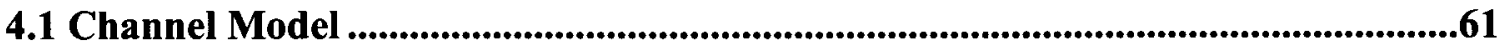

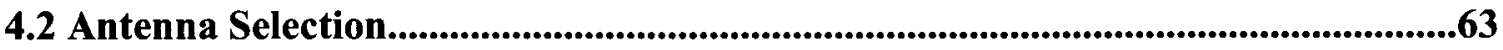

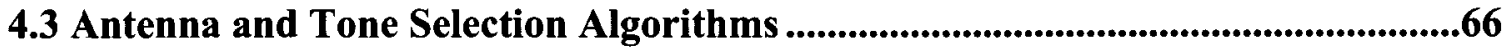

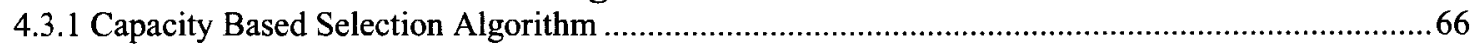

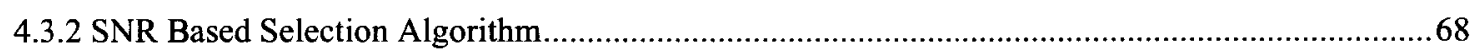

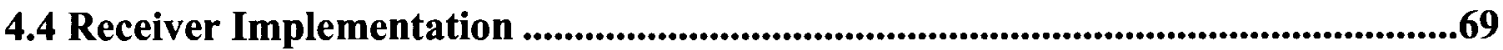

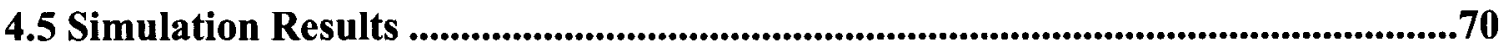

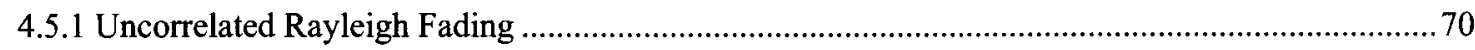


5 Decorrelation of Multi-user Downlink MIMO Channels ............................... 84

5.1 Channel Model .....................................................................................................................85

5.2 Downlink Null Steering Signaling Models................................................................87

5.2.1 Signal Model of a Single Antenna Mobile Users (downlink MISO) .....................................8

5.2.2 Signal Model of a Multi Antenna Mobile Users (downlink MIMO) ....................................89

5.3 Decorrelation Algorithm ................................................................................................93

5.4 Simulation Results and Discussion ................................................................................94

6 Decorrelation of Uplink Multi-user MIMO Channels ................................. 106

6.1 Adaptive Filters and the Eigen Structure of the Wireless Channel .....................106

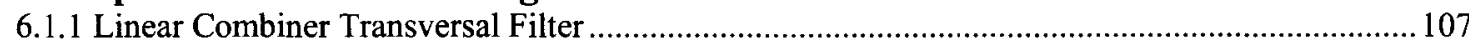

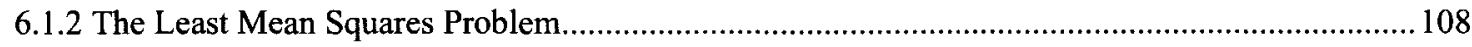

6.2 Decorrelation of an Adaptive Uplink Signaling Scheme .......................................110

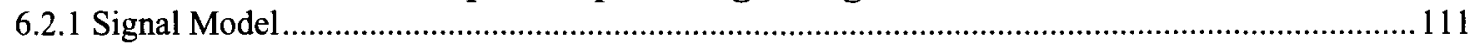

6.3 Decorrelation of a Space-time Block Coded Uplink Signaling Scheme................113

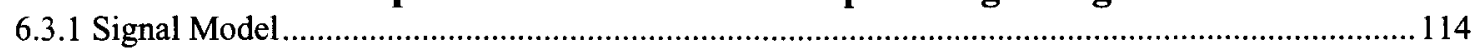

6.4 Simulation Results ..........................................................................................................116

6.4.1 Simulation Results of the Adaptive Signaling Scheme....................................................116

6.4.2 Simulation Results of the Space-time Block Coded Signaling Scheme................................. 121

7 Conclusions and Future Work................................................................ 125 


\section{Abbreviations and Acronyms}

\begin{tabular}{|c|c|}
\hline AoA & Angle of arrival. \\
\hline AoD & Angle of departure. \\
\hline AWGN & Additive white Gaussian noise. \\
\hline BER & Bit error rate. \\
\hline BS & Base station. \\
\hline $\mathrm{CDF}$ & Cumulative distribution function. \\
\hline $\mathrm{CP}$ & Cyclic Prefix. \\
\hline CSI & Channel state information. \\
\hline $\mathrm{DAB}$ & Digital audio broadcasting. \\
\hline DFT & Discrete Fourier transform. \\
\hline DSP & Digital signal processing \\
\hline DVB & Digital video broadcasting. \\
\hline $\mathrm{E} / \mathrm{O}$ & Electrical / optical. \\
\hline ICI & Inter-carrier interference. \\
\hline IDFT & Inverse Discrete Fourier Transform. \\
\hline IFFT & Inverse fast Fourier transform. \\
\hline ISI & Inter Symbol Interference. \\
\hline LAN & Local area network. \\
\hline LMS & Least mean square. \\
\hline LOS & Line of sight. \\
\hline MAC & Media access control. \\
\hline MIMO & Multiple-input multiple-output. \\
\hline MISO & Multiple-input single-output. \\
\hline MMSE & Minimum mean square error. \\
\hline MRC & Maximum ratio combining. \\
\hline MS & Mobile station. \\
\hline $\mathrm{O} / \mathrm{E}$ & Optical/electrical. \\
\hline OFDM & Orthogonal frequency division multiplexing. \\
\hline OFDMA & Orthogonal Frequency Division Multiple Access. \\
\hline PAPR & Peak-to-average power ratio. \\
\hline
\end{tabular}


PDP

PSK

QAM

QoS

RF

RoF

SDMA

SIMO

SINR

SISO

SNR

SVD

TDMA

$\mathrm{WiFi}$

WiMAX

WLAN

ZF

Power delay profile.

Phase shift keying.

Quadrature amplitude modulation.

Quality of service.

Radio frequency.

Radio over fiber.

Space division multiple access.

Single-input multiple-output.

Signal to interference plus noise ratio.

Single-input single-output.

Signal to noise ratio.

Singular value decomposition.

Time division multiple access.

Wireless Fidelity.

Worldwide Interoperability for Microwave Access.

Wireless Local Area Networks.

Zero forcing. 


\section{Notations and symbols}

\begin{tabular}{|c|c|}
\hline $\mathbf{G}$ & Time domain channel matrix. \\
\hline $\mathbf{H}_{k}$ & Channel matrix of the $k^{\text {th }}$ tone. \\
\hline $\mathbf{R}_{R}$ & Spatial correlation matrix at the receiver. \\
\hline $\mathbf{W}$ & Beamforming matrix at the transmitter. \\
\hline $\mathbf{y}$ & Vector of received samples over the $k^{\text {th }}$ tone. \\
\hline $\mathbf{s}_{k}$ & Vector of transmitted symbols over the $k^{t h}$ tone. \\
\hline$s$ & Transmitted symbol. \\
\hline $\mathbf{R}_{\text {ss }}$ & Covariance matrix of the transmitted vector $\mathbf{s}$ \\
\hline $\mathbf{a}$ & Vector $\mathbf{a}$. \\
\hline $\mathbf{A}$ & Matrix A. \\
\hline $\mathbf{A}^{H}$ & Hermitian conjugate of matrix $\mathbf{A}$. \\
\hline $\mathbf{a}^{T}$ & Transpose of vector $\mathbf{a}$ \\
\hline $\operatorname{diag}(\mathbf{a})$ & Diagonal matrix with vector $\mathbf{a}$ in its diagonal. \\
\hline $\operatorname{rank}(\mathbf{A})$ & Rank of matrix $\mathbf{A}$. \\
\hline $\operatorname{Tr}(\mathbf{A})$ & Sum of elements in the diagonal of matrix $\mathbf{A}$. \\
\hline$E\{\mathbf{A}\}$ & Expectation of matrix $\mathbf{A}$. \\
\hline$\|\mathbf{A}\|^{2}$ & Frobenius norm of the matrix $\mathbf{A}$. \\
\hline$n_{B}$ & Number of spatial subchannels. \\
\hline$n_{T}$ & Number of transmit antennas. \\
\hline$n_{R}$ & Number of receive antennas. \\
\hline$n_{P}$ & Number of antenna arrays. \\
\hline$n_{L}$ & Number of antennas within each array. \\
\hline$n_{C}$ & Number of OFDM tones. \\
\hline$n_{K}$ & Number of selected OFDM tones. \\
\hline$n_{U}$ & Number of mobile users in the microcell. \\
\hline$n_{S}$ & Number of selected users in each OFDM tone. \\
\hline$n_{D}$ & Number of symbols transmitted to each user ove \\
\hline
\end{tabular}




\begin{tabular}{|c|c|}
\hline$n_{V}$ & Number of diversity branches. \\
\hline$L_{T}$ & Number of selected antennas at the transmitter. \\
\hline$L_{R}$ & Number of selected antennas at the receiver. \\
\hline$L$ & Number of channel taps. \\
\hline$P L$ & Path Loss. \\
\hline$P L_{0}$ & The pathloss at a distance of $d_{0}$. \\
\hline$K_{p}$ & $K$-factor at the $p^{\text {th }}$ array. \\
\hline$\gamma$ & The pathloss exponent. \\
\hline$r m s$ & Root mean square. \\
\hline$\theta_{R}$ & The angle of arrival. \\
\hline$\alpha$ & Angular spread. \\
\hline$P_{R}\left(\theta_{R}\right)$ & Angular spectrum at the receive array. \\
\hline$D_{R}$ & Antenna spacing of the receive array. \\
\hline$\Delta_{R}$ & Receive antenna separation normalized to the carrier wavelength. \\
\hline $\mathbf{e}_{R}\left(\theta_{R}\right)$ & Unit spatial signature at the receive array. \\
\hline$c$ & The speed of light. \\
\hline$f_{c}$ & Carrier frequency. \\
\hline$\lambda_{c}$ & Carrier wavelength. \\
\hline$\beta_{\mathrm{LOS}, p}$ & Shadowing component of the LOS channel at the $p^{\text {th }}$ array. \\
\hline$E_{s}$ & Symbol energy. \\
\hline$\lambda_{i}$ & $i^{t h}$ eigenvalue. \\
\hline$C_{\text {out }}$ & Outage capacity. \\
\hline$C$ & Channel capacity. \\
\hline $\mathbf{I}_{n_{R}}$ & Identity matrix of size $n_{R} \times n_{R}$ \\
\hline$\rho$ & Average received SNR of a SISO link. \\
\hline
\end{tabular}




\section{List Of Tables}

TABLE 4.1. AN EXAMPLE OF THE ANTENNAS ASSIGNED TO A USER IN FOUR ASSIGNED OFDM TONES...69

TABLE 5.1. NUMBER OF OFDM TONES ALLOCATED TO EACH OF THE 9 USERS (OUT OF 64 TONES),

WITH $N_{P}=8, N_{L}=2, N_{R}=3, N_{U}=9, N_{S}=4, \mathrm{SNR}=15 \mathrm{DB}$. .102 


\section{List Of Figures}

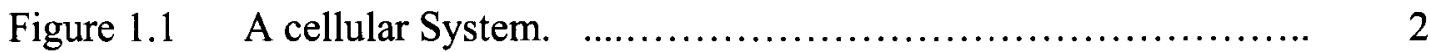

Figure $1.2 \quad$ Multipaths in a wireless channel. ............................ 4

Figure 1.3 Effect of fading of wireless channel on the received signal amplitude

Figure 1.4 Capacity of a white channel versus signal to noise ratio for different antenna configurations, with unknown CSI at the transmitter.

Figure 1.5 A propagation scenario of a base-station system employing an SDMA technique to serve many mobiles using nullsteering. ..... 9

Figure 1.6 Beam steering and null steering beamformers.................. 10

Figure 1.7 Intensity modulation direct detection (IMDD) analog optical link. .................................................... 17

Figure 2.1 Wave front impinging on a two element antenna array. ......... 25

Figure $2.2 \quad$ Line of sight SIMO channel. ............................. 27

Figure $2.3 \quad$ Line of sight MIMO channel. ........................... 30

Figure 2.4 Two transmit and three receive clusters of waves. ............. 32

Figure 2.5 Multi-users in a correlated Ricean channel with distributed basestation antennas. ...................................... 34

Figure 2.6 Baseband OFDM link. ................................ 38

Figure 3.1 Ergodic capacity of an uncorrelated channel for different antenna configurations, with unknown CSI at the transmitter. ............. 44

Figure 3.2 Ergodic and 15\% outage capacities of an uncorrelated channel. .. 45

Figure 3.3 Available spatial subchannels between the transmitting and receiving arrays.

Figure 3.4 A single antenna transmitting to an array of $n_{R}$ antennas. ....... 49

Figure $3.5 \quad$ Alamouti signaling scheme. ............................. 51 
Figure 3.6 CDF of channel capacity with different number of selected Basestation antennas...................................... 56

Figure 3.7 Effect of the $K$-factor on the MIMO channel capacity. .......... 58

Figure 3.8 Decrease in ergodic capacity due to increase in channel correlation.

Figure 4.1 Distributed antenna arrays with a single mobile user.

Figure 4.2 OFDM tones transmitted from different antennas.

Figure 4.3 Two distributed antennas transmitting the same OFDM tone to a mobile user.

Figure 4.4 CDF of channel capacity for different antenna configurations $(\mathrm{SNR}=10 \mathrm{~dB})$.

Figure 4.5 BER versus SNR of different antenna configurations using zeroforcing and MMSE receivers.

Figure 4.6 CDF of channel capacity for different antenna configurations, $\mathrm{SNR}=10 \mathrm{~dB}, K_{P}=10$.

Figure 4.7 CDF of channel eigenvalues for different antenna configurations.

Figure 4.8 CDF of channel condition number for different antenna configurations, $\mathrm{SNR}=10 \mathrm{~dB}$. $\mathrm{K}_{\mathrm{P}}=10$, fixed correlation.

Figure 4.9 Ergodic capacity versus SNR for different antenna configurations.

Figure 4.10 CDF of channel capacity under different antenna configurations, and different correlation and Ricean conditions.

Figure 4.11 CDF of condition number under different antenna configurations, and different correlation and Ricean conditions.

Figure 4.12 Ergodic capacity versus SNR under different channel environments.

Figure 5.1 BER performance of a MISO propagation scenario, before and after the distribution of the basestation antennas, with $D_{\min }=60$, $D_{\max }=2000$.

Figure 5.2 BER performance of a MISO propagation scenario, before and after the reduction of inter-user cross-correlations, with $n_{P}=12$, $n_{L}=1$.

Figure 5.3 System BER performance with co-located basestation antennas and different correlation scenarios, without correlation reduction, with $n_{P}=1, n_{L}=16, n_{R}=3, n_{U}=5, n_{S}=5$.

Figure 5.4 BER performance of system with distributed basestationantennas, without using cross-correlation reduction algorithm, with $n_{P}=8, n_{L}=2, n_{R}=3, n_{U}=5, n_{S}=5$. 
Figure 5.5 User BER performance with distributed basestation antennas, using the cross-correlation reduction algorithm, with $n_{P}=8, n_{L}=$ $2, n_{R}=3, n_{U}=9, n_{S}=5$.

Figure 5.6 User BER performance with more correlated users, using the cross-correlation reduction algorithm, with $n_{P}=8, n_{L}=2, n_{R}=3.101$

Figure 5.7 Probability mass function of the number of tones allocated to each user, with $n_{P}=8, n_{L}=2, n_{R}=3, n_{U}=9, n_{S}=4, \mathrm{SNR}=15 \mathrm{~dB}$.

Figure 5.8 Effect of the near-far problem on the BER for different antenna configurations and different mobile users' distances to the arrays. 104

Figure 5.9 Effect of water pouring on the average BER, with $n_{P}=8$, $n_{L}=3, n_{R}=2, n_{U}=8, n_{S}=4$.

Figure 6.1 Linear combiner transversal adaptive filter.

Figure 6.2 Mobile users transmitting to distributed basestation antennas. ...

Figure 6.3 Training symbols transmitted from multiple users to a distributed basestation antennas employing adaptive filter. .....

Figure 6.4 CDF of eigenvalues and condition number with near-far problem and co-located basestation antennas as $n_{P}=1, n_{L}=32, \mathrm{D}_{\min }=$ $70 \mathrm{~m}, \mathrm{D}_{\max }=1000 \mathrm{~m}$.

Figure 6.5 CDF of eigenvalues and condition number for with a near-far problem and distributed antennas as $n_{P}=8, n_{L}=4$, and $n_{P}=16$, $n_{L}=2$.

Figure 6.6 Effect of antenna configuration on the MSE of several mobile users with intra-user correlations.

Figure 6.7 MSE of $n_{U}=5$ users with inter-user correlations without using decorrelation algorithm.

Figure 6.8 MSE of $n_{U}=9$ users with inter-user correlations when selecting $n_{S}=5$ users, in each OFDM tone, using the decorrelation algorithm.

Figure 6.9 Effect of distributing the basestation antennas on the system performance.

Figure 6.10 Effect of inter-user correlation reduction on average bit error rate.

Figure A.1 Block diagram of a narrow-band adaptive beamformer antenna. .

Figure A.2 A propagation scenario of a base-station system employing an SDMA technique to serve many mobiles using nullsteering. ..... 131

Figure A.3 Delay and sum beamformer. 


\section{Chapter 1}

\section{Introduction and Motivation}

\subsection{Wireless Communication Systems}

Wireless systems are being implemented throughout the world to meet the increasing consumer demand. Cellular mobile systems, wireless local area networks (LAN) and digital cordless telephony are examples of such systems.

Cellular mobile systems are growing at about $40 \%$ per year and are expected to continue in this trend for several years. In cellular mobile systems, the area to be served is divided into several cells and each cell has a basestation, connected to a mobile telephone switching office, that serves several mobile users (Figure 1.1). The basestation transmits to the users in the downlink transmission while in the uplink, the mobile users transmit to the basestation [1].

Wireless LANs (WLANs) are also spreading at a tremendous speed nowadays. They are easy to install, scalable and have flexible configurations which can be as simple as peer-to-peer topologies, or complex multiuser networks. Examples are the home wireless Internet routers and Wireless Fidelity (WiFi) technology, a recent wide-spreading WLAN that uses specifications conforming to the IEEE $802.11 \mathrm{~b} / \mathrm{g}$ standards. WiFi has gained acceptance in many environments as an alternative to a wired LAN. Many airports, hotels, and other services offer public access to WiFi networks so people can log onto the Internet and receive emails on the move. These locations are known as hotspots.

Another new standard is worldwide interoperability for microwave access (WiMAX) which is a wireless technology that provides high-throughput broadband connections over long distances [2]. WiMAX is based on the IEEE 802.16d air interface standard and is used for fixed broadband wireless access systems employing a point-to-multipoint architecture. WiMAX can be used for a number of applications, including "last mile" broadband connections, hotspots and cellular backhaul, and high-speed enterprise 


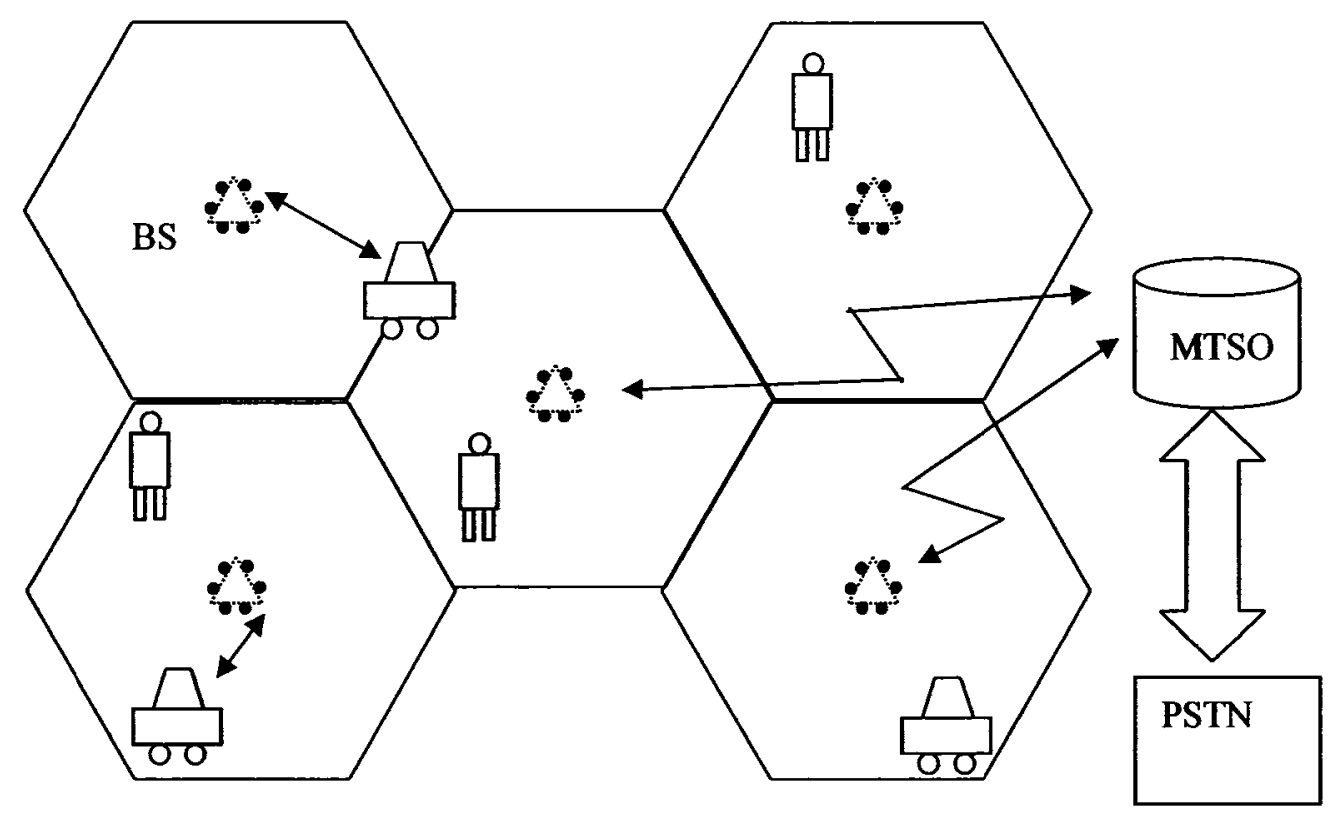

Figure 1.1. A cellular system.

connectivity for business. The so-called "last mile" of broadband is the most expensive and most difficult for broadband providers, and WiMAX provides an easy solution.

Because of the huge market demand, wireless communication has seen a surge of research activities during the last two decades. The tremendous demand for high data rate cellular telephony in particular and for wireless applications in general is one factor that ignited this activity. Another factor is the explosive improvement in VLSI technology which enabled the implementation of sophisticated signal processing algorithms and coding techniques in small-size, low-power communication devices.

Wireless communications, in contrast to wireline communications, is faced with many challenging problems such as fading and interference. Small-scale fading, due to multipath signal propagation, and large-scale fading, due to pathloss and shadowing, both cause a time variation of the power transfer gain of the wireless channel. Interference can be classified as intra-cell interference, between users and the basestation within the same cell, and inter-cell interference, between users and basestations in different cells. 
To briefly understand fading, consider a wireless channel with one transmitting and receiving antenna (Figure 1.2). The transmitted signal will arrive at the receiving antenna through a number of distinct paths. These paths are caused by signal reflections and scattering off fixed objects such as buildings, hills, trees and moving objects such as vehicles and people. Each of these paths has a distinct delay, angle of arrival and gain. These paths will be added at the receiving antenna constructively or/and destructively and can cause the received signal to vary as a function of frequency, time and space. These variations are called fading. Figure 1.3 shows the typical received signal power over time. It can be seen that the signal level may experience deep nulls leading to an unreliable wireless link.

Due to the above mentioned problems, wireless systems are power, bandwidth and complexity limited which results in less capacity compared to wireline systems. Multiple-input multiple-output (MIMO) systems, and orthogonal frequency division multiplexing (OFDM), are two techniques that, if appropriately combined, have the potential to provide data transmission with very high spectral efficiency and thereby meet the high speed requirement of future wireless systems.

\subsection{MIMO Systems and Achievable Gains}

By employing multiple-input (transmit) and multiple-output (receive) antennas, MIMO communication systems can exploit the space domain to significantly increase the system capacity over single-input single-output (SISO) systems. Research in this area became more intense after the pioneering work by Foschini [3] and Telatar [4] that predicted significant spectral efficiencies for wireless MIMO systems when the channel exhibits rich scattering and is known to one or both ends of the wireless link.

The large capacity achieved from MIMO systems makes them an ideal candidate for future wireless systems. This capacity increase is considered "for free", compared to SISO systems, as it is achieved without additional power or bandwidth and is mainly due to spatial multiplexing gain in addition to diversity and array gains. Various parameters determine the amount of gain that can be achieved from a MIMO system, including, for 


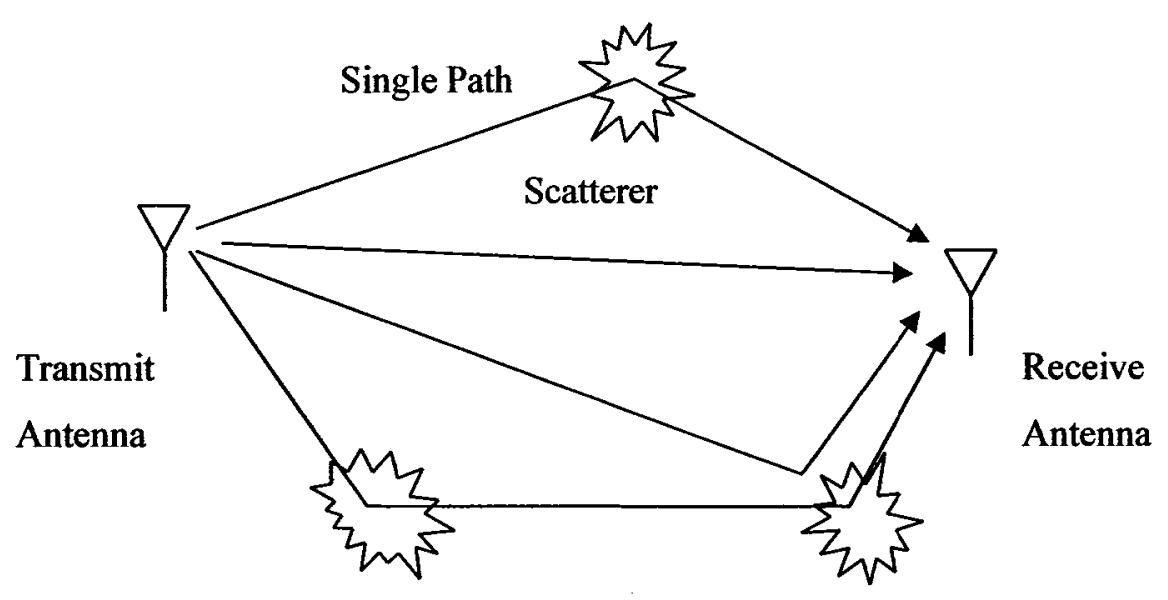

Figure 1.2. Multipaths in a wireless channel.

example, system structure, the transmit and receive strategies, the channel properties and the accuracy of the channel state information (CSI) at the transmitter and the receiver. In order to capture array and diversity gains, the knowledge of the CSI at the receiver and/or the transmitter is usually required [5]. Without the knowledge of the CSI at the transmitter, spatial multiplexing gain can be achieved using space-time block coding or space-time trellis coding [6][7][8], while with knowledge of the CSI at the transmitter spatial multiplexing gain can be optimally achieved using eigen beamforming.

\subsubsection{Array Gain}

Array gain leads to an increase in average signal to noise ratio (SNR) at the receiving antennas due to coherent combining of the signals received at each antenna. Array gain can be achieved at the receiving antennas by matching the phases of the received signals at the antennas then adding them (i.e. coherent combining). If the CSI is known at the transmitter, array gain can also be achieved from the transmitting antennas by adjusting 


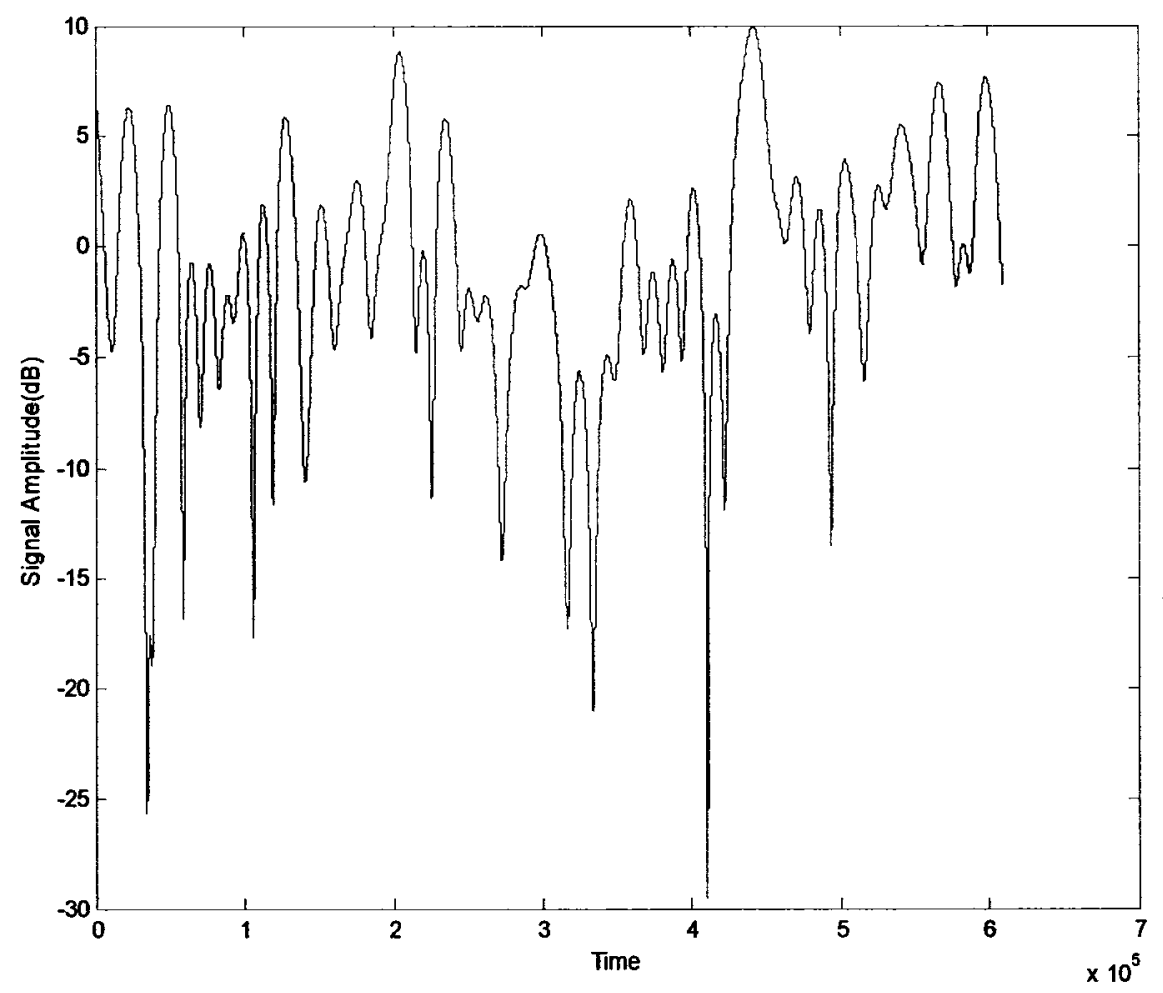

Figure 1.3. Effect of fading of wireless channel on the received signal amplitude.

the phases of the transmitted signals such that they arrive coherently (in-phase) to each receiving antenna [9].

With channel knowledge at both the transmitter and the receiver, array gain can be obtained at both ends of the wireless link. Array gain can be viewed as an enhancement of antenna gain by adjusting the look direction (power pattern) of the antenna array towards the target instead of wasting the power pattern of the array evenly towards all directions (i.e. isotropic antenna). 


\subsubsection{Diversity Gain}

Because the power of the signal in a wireless channel fluctuates due to fading, diversity is used to reduce or combat this fluctuation. For example, in a system where the receiver has multi-antennas, the receiver may see (depending on different environmental and/or system conditions) independently faded copies of the same transmitted signal. The number of these copies depends on the available number of diversity branches between the transmitter and the receiver. It is unlikely that all branches are heavily attenuated simultaneously. The receiver will combine these received independent signals such that the resulting signal will have reduced amplitude fluctuations.

The number of these independent branches is called the diversity order. These branches might be in the time, frequency and/or space domains. The actual realized diversity order depends on the design of the transmitter and/or receiver. Good design will exploit all available diversity. Using space-time diversity coding, for example, the maximum diversity order is possibly obtained with or without channel knowledge at the transmitter but array gain cannot be obtained at transmitter without knowledge of CSI at the transmitter [10].

\subsubsection{Spatial Multiplexing Gain}

The gain in ergodic capacity, over SISO systems, resulting from the use of multiple antennas is called the spatial multiplexing gain. Spatial multiplexing means multiplexing different transmitted signals simultaneously in the same frequency band [6][11][12]. While increasing diversity results in an increase in average received SNR, spatial multiplexing is a technique that increases the data rate of the system. In spatial multiplexing, more than one symbol can be transmitted in each channel use. Spatial multiplexing is possible only in cases of multiple antennas at both ends of the wireless link [3][13].

The main reason behind the huge spectral efficiency of MIMO channels is that, beside the increase in spatial diversity order, the rich scattering environment around the transmitting and receiving antennas provides several independent transmission paths 
(spatial subchannels) between the transmitting and receiving antenna arrays. The number of these paths is equal to the rank of the matrix representing the channel gains between the transmitting and receiving antenna arrays (the channel matrix). Therefore, for a system with $n_{T}$ transmitting antennas and $n_{R}$ receiving antennas, up to $n_{B}=\min \left(n_{T}, n_{R}\right)$ independent paths can each carry one data symbol in each channel use [4][29]. Thus the channel capacity increases linearly with the same factor $\left(n_{B}\right)$ compared to a SISO wireless link. This capacity increase is conditional upon the existence of a wireless channel with a rich scattering environment that makes the channel matrix of full rank and uncorrelated entries and on the knowledge of CSI at the receiving side and/or transmitting side of the wireless link. Figure 1.4 shows the increase in ergodic channel capacity with the increase of the number of antennas at both ends of an uncorrelated Rayleigh channel.

Space-time signal processing can permit the multiple antennas at the basestation to communicate with several spatially dispersed mobile users. This is another form of spatial multiplexing where multiple data symbols are simultaneously transmitted, and on the same frequency band, to serve multiple users. In the uplink transmission, with CSI knowledge at the basestation, signals of different users can be distinguished at the basestation based on the different spatial signatures of each user. In the downlink case, pre-processing of users signals enables the transmission of several data symbols simultaneously to each user with minimum inter-user interference. This is the so called space division multiple access (SDMA) [14][15].

Using multiple antennas, inter-user interference can be greatly reduced. For example, unwanted mobile users can be nulled by the basestation and most of the power can be directed towards the desired mobile user(s) (see Figure 1.5). The importance of SDMA in wireless is not only because of its multiple access capability, but also because it allows channel reuse within the cell, resulting in a significant increase in spectral efficiency [15][16].

Several SDMA signaling schemes have been proposed in numerous references. Nishimura et al. compared two downlink beamforming methods (Figure 1.6), a general beamformer, which maximizes the signal transmitted to the desired user (i.e. maximize 


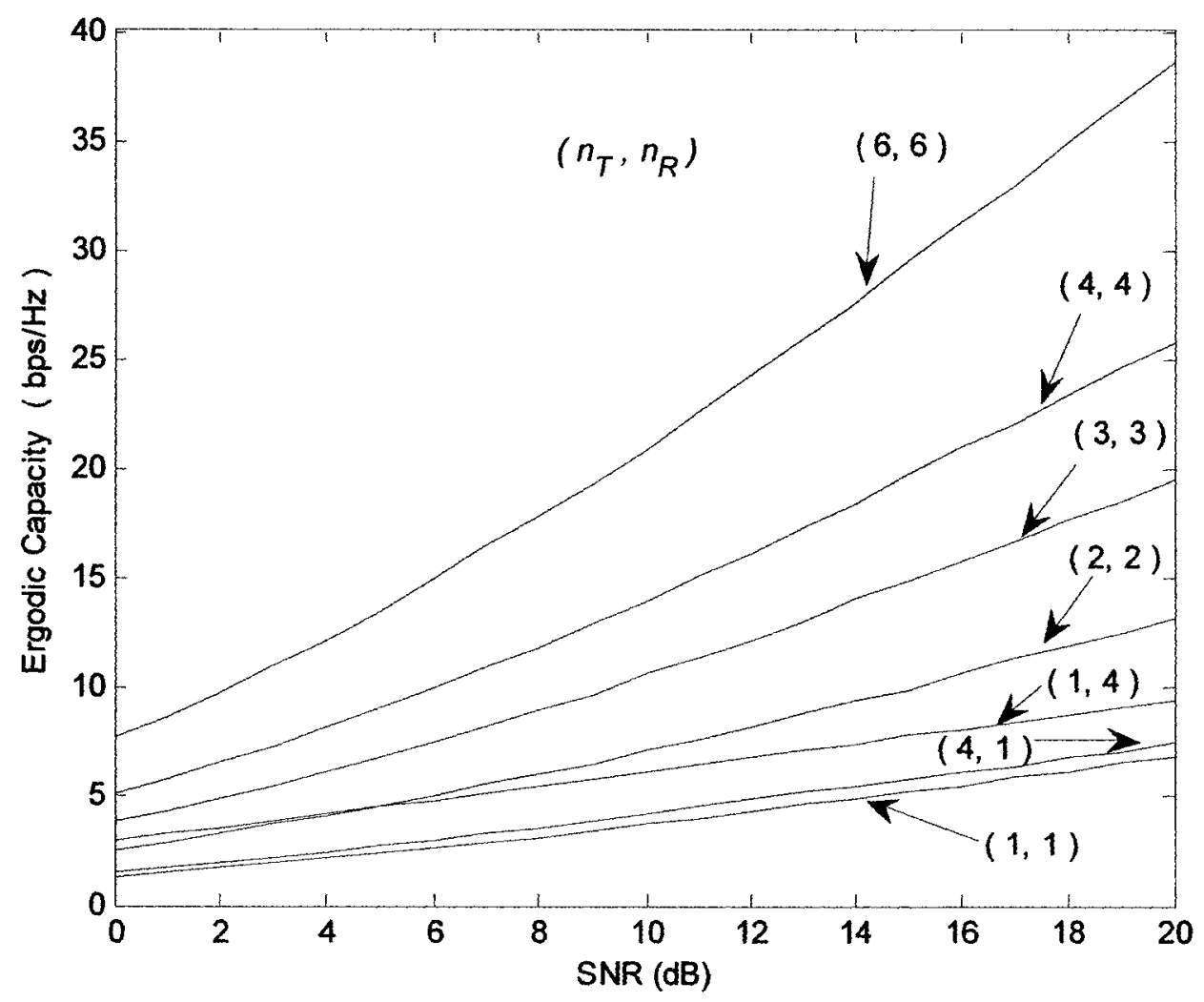

Figure 1.4 Capacity of a Rayleigh channel versus signal to noise ratio for different antenna configurations, with unknown CSI at the transmitter.

the power pattern of the basestation antennas toward the desired user) and a null steering beamformer that ensures that a signal transmitted to a user is nulled to all other users. Computer simulations results show that the null steering scheme yields better performance than the other scheme even when using receive MRC [17].

In another work by Nishimura et al, a downlink zeroforcing SDMA technique is proposed that transmits a single data symbol to each of the two co-existing mobile users [18]. The technique selects one antenna (out of the two user's antennas) that has the 


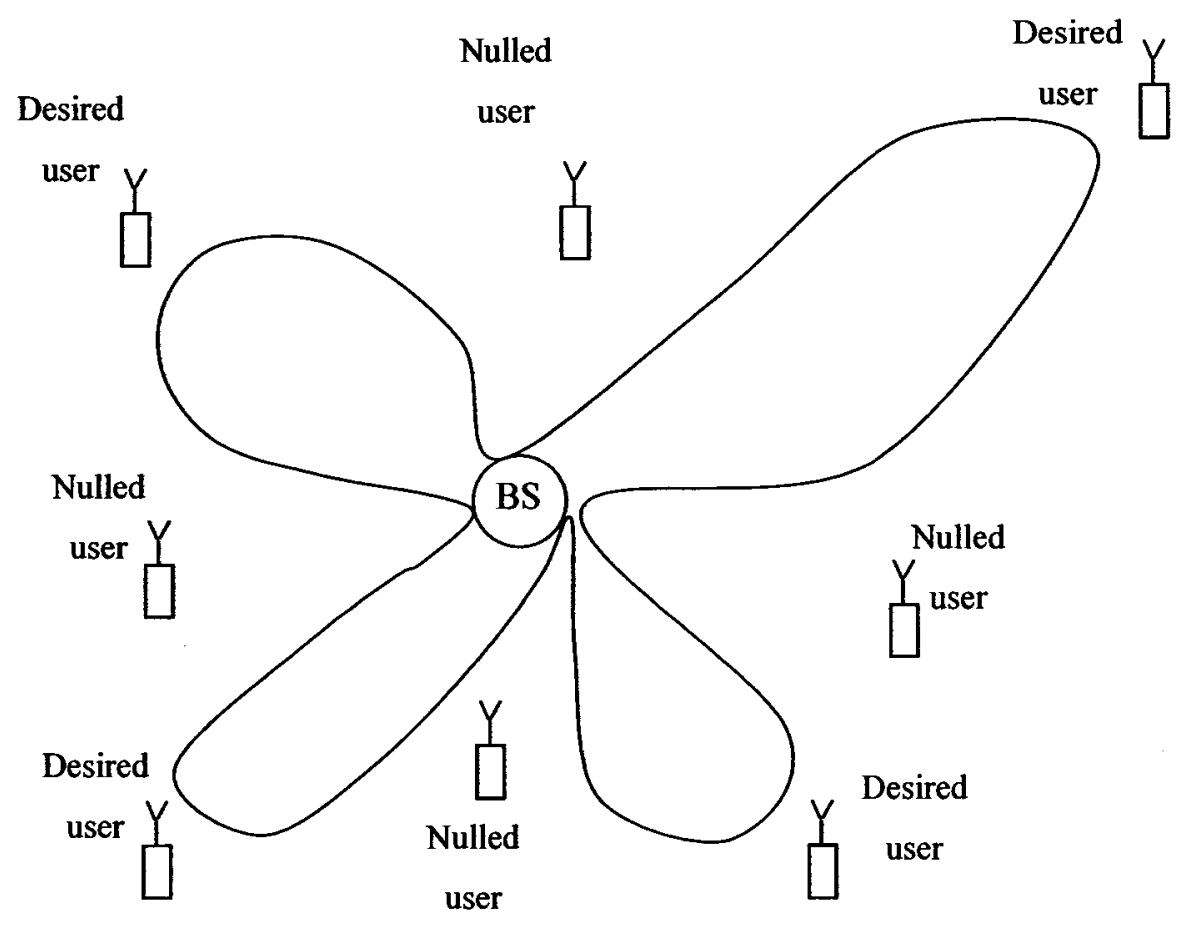

Figure 1.5. A propagation scenario of a base-station system employing an SDMA technique to serve many mobiles using nullsteering.

lowest spatial correlations. A signal transmitted to one user's antenna is nulled on the other user's selected antenna. This technique is simple but the achieved system throughput is relatively low, since it is a MISO rank one channel and it is valid for two users only.

A different nullsteering technique was proposed by Rim et al. [19]. It is a downlink SDMA with $n_{T}$ antennas at the basestation and $n_{R}$ at each of the $n_{U}$ mobile stations. The channel is assumed to be quasi-stationary flat fading with a rich scattering environment. It is also assumed that the CSI is known at the transmitter (basestation). Computer simulations showed that the proposed technique, which can transmit multiple data streams to each user, achieved much higher downlink channel capacity than conventional 


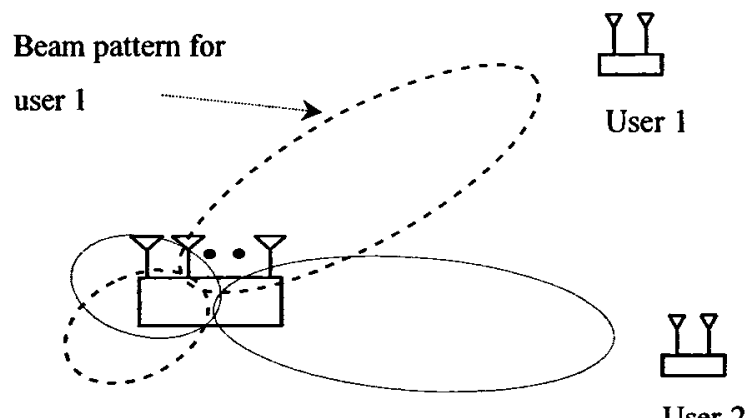

(a) Beam steering method

User 2

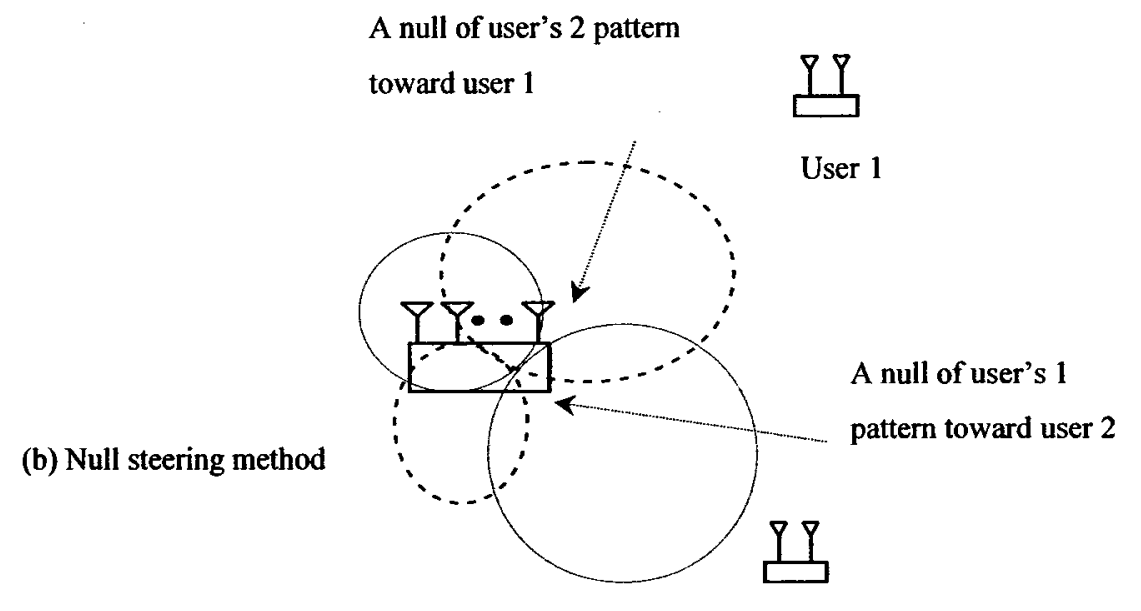

User 2

Figure 1.6. Beam steering and null steering beamformers.

single user MIMO techniques such as open loop MIMO (where CSI is unknown at the transmitter) and singular value decomposition MIMO. The whole process is complicated and cumbersome as the number of users and antennas is increased and it assumes uncorrelated users channel matrices.

In addition to the above SDMA techniques, Choi and Murch introduced a downlink pre-processing technique that decomposes multi-user uncorrelated MIMO flat fading channels into multiple parallel independent single-user MIMO downlink channels [20]. Spencer et al. presented a technique that diagonalizes a multi-user MIMO channel such 
that each multi-antenna user can receive multiple data streams in each channel use [21]. Appendix A gives a brief review of beamforming and SDMA techniques.

\subsection{MIMO-OFDM Wireless Systems}

Orthogonal frequency division multiplexing (OFDM) is a modulation technique in which a wide band signal is divided into several orthogonal narrow signals (subcarriers or tones) and all are transmitted in parallel (multiplexed in the frequency domain). OFDM allows the spectrum of each tone to overlap, and because they are orthogonal, they do not interfere with each other. The information is modulated onto a tone by adjusting the tone's phase, amplitude, or both. In the most basic form, a tone may be on (used) or off (unused) to indicate a one or a zero bit of information, however, either phase shift keying (PSK) or quadrature amplitude modulation (QAM) is typically employed. The OFDM system splits the input data stream into $N$ parallel data streams each at a rate of $1 / N$ of the original rate. Each stream is then mapped to a tone at a unique frequency and combined together using the inverse fast Fourier transform (IFFT) to yield the time-domain waveform to be transmitted.

The main reason for using OFDM is that OFDM modulation turns a frequencyselective fading channel into a set of parallel frequency-flat fading channels. This simplifies multi-channel equalization, since for every OFDM tone a narrow-band receiver can be employed.

OFDM can also be used as a multiple-access technique, because an individual tone or groups of tones can be assigned to different users. Multiple users share a given bandwidth in this manner, yielding the system called orthogonal frequency division multiple access (OFDMA). Each user can be assigned a predetermined number of tones when they have information to send, or alternatively, a user can be assigned a variable number of tones based on the amount of information they have to send. The assignments are controlled by the media access control (MAC) layer, which schedules the resource assignments based on user demand [22]. The new WiMAX standard (IEEE 802.16 d,e) is an OFDMA based technology. 
Using OFDM in a MIMO system provides a set of parallel frequency flat MIMO channels, and spatial multiplexing and SDMA can be applied independently over each of these channels [23][24]. However, there are several important issues that need to be addressed for MIMO-OFDM systems to be effective. These include, amongst others, channel estimation, unequal error protection in OFDM, power gain imbalances, and correlations between channel matrix elements.

\subsubsection{Channel Estimation}

MIMO system capacity is greatest when accurate CSI is available at both the transmitter and receiver, enabling the use of transmit beamforming and the corresponding receive signal processing. The ability to accurately estimate the channel at the receiver and feed this information back to the transmitter is therefore an important system requirement.

One of the most popular approaches to downlink channel estimation is channel probing with user feedback. This approach suggests to probe the downlink channel by transmitting training signals from the base station to each user and then to estimate the channel from feedback reports provided by the users. When OFDM is used over a frequency selective fading MIMO channel, the channel has to be estimated for each subcarrier by distributing pilot tones across the subcarriers (and across OFDM symbols, for time varying channels) with certain spacing and then interpolation is performed to estimate the channels that do not carry pilot tones.

To be able to interpolate the channel estimates both in time and frequency from the available pilots, the pilot spacing has to fulfill the Nyquist sampling theorem, which states that the sampling interval must be smaller than the inverse of the double-sided bandwidth of the sampled signal. For the case of OFDM, this means that there exists both a minimum subcarrier spacing and a minimum symbol spacing between pilots. By choosing the pilot spacing much smaller than these minimum requirements, good channel estimation can be made with a relatively easy algorithm. The more pilots are used, however, the smaller the effective SNR that becomes available for data symbols. Hence, the pilot density is a tradeoff between channel estimation performance and SNR loss [22]. 
For a quasi static channel, the packet length is short enough to assume a constant channel during the length of the packet. This means there is no need to estimate time fading, which greatly simplifies the channel estimation problem. The other advantage of channel estimation across the tones only is to avoid the delay of several symbols before the first channel estimates can be calculated, from the pilots scattered over several OFDM data symbols. Any delay in the reception of a packet will also delay the acknowledgment and hence decrease the effective throughput of the system. Such a delay is undesirable in packet transmission like in an IEEE 802.11a wireless LAN, which requires an acknowledgment to be sent after each packet transmission. For the specific problem of channel estimation in packet transmission systems, the most appropriate approach seems to be the use of a preamble consisting of one or more known OFDM symbols. The packet starts with two OFDM symbols for which all data values are known. These training symbols can be used to obtain channel estimates, as well as a frequency offset estimate.

\subsubsection{Unequal Error Protection}

In a frequency selective fading channel, the subcarriers of the OFDM symbol will arrive at the receiving antennas with different amplitudes. In fact, some subcarriers may be completely lost because of deep fades. Hence, even though most subcarriers may be detected without errors, the overall bit error rate (BER) will be largely dominated by a few subcarriers with the smallest amplitudes, for which the BER is very high. To avoid this domination by the weakest subcarriers, forward error correction coding is essential. In channel coding, redundancy is introduced into the transmitted data. This redundancy can be exploited, at the receiver, to protect the information being transmitted over the channel by correcting channel errors. By using coding across the subcarriers, errors of weak subcarriers can be corrected up to a certain limit that depends on the strength of the code used and the channel properties. A powerful coding means that the performance of an OFDM link is determined by the average received power, rather than by the power of the weakest subcarrier. 


\subsubsection{Antenna Selection}

Complexity and cost are significant problems of any MIMO system due to the requirement of a large number of complete radio frequency (RF) chains. This complexity is sometimes undesirable especially for the mobile station because RF elements can be a significant cost factor. For this reason there is a great interest in antenna selection schemes [25].

By using only a small subset of available antennas, the number of RF chains can be reduced, decreasing system complexity and cost, with only a small performance loss compared to a full-complexity system [26].

\subsubsection{Power Gain Imbalance}

Because the mobile users are usually located at different distances from the basestation, a near-far problem is created which in turn makes the channel matrix seen by the basestation to all served mobile users heavily "unbalanced". Such an unbalanced channel matrix has a high eigenvalue spread (rank inefficient) which results in low total channel capacity. In an uplink scenario, the strong signal from a nearby user may overwhelm the weak signal from a distant user, thereby reducing capacity. Transmit power control strategies are helpful in compensating for this imbalance.

\subsubsection{Channel Matrix Correlations}

The correlations within the MIMO channel matrix are an important factor governing the capacity of the wireless channel. They depend heavily on the scattering environment, antenna configuration, angular spread, angular spectrum and the angles of arrival and departure of the waves. In SDMA systems, when two or more users are close to each other or when the scattering environment is in such a way that makes the spatial signatures of the users almost identical, then the channel matrices of these users will become highly correlated (inter-user correlations). This makes user separability very difficult if not impossible, which will become a source of outage or link failure. 
Large channel matrix correlations may decrease the number of non-zero eigenvalues and increase the eigenvalues spread. This in turn reduces the number of available spatial subchannels, which is the rank, and also greatly varies the gains of these subchannels which leads to an increase of the condition number of the channel matrix. This is a reduced rank scenario with a high eigenvalue spread which results in a significant reduction of the channel capacity [27][28].

While the mobile user is usually immersed in a rich scattering environment, the basestation antennas are usually located on a tower with no nearby scatterers. This decreases the angular spread and may, consequently, increase the channel matrix correlations. A very significant multiplexing gain of MIMO systems is achieved when the basestation antennas are immersed in a scattering environment [27][29].

Line of sight (LOS) conditions often create undesirable MIMO conditions (i.e. high condition number). When the basestation antennas are located at the top of the basestation tower, the LOS condition is expected. The ability of the mobile user antennas to resolve different transmitted basestation symbols decreases with the existence of LOS (a non-scattering environment) [27][29].

\subsection{Thesis Overview}

Although all of the above issues are essential for MIMO-OFDM systems, in this thesis we limit our focus primarily to the channel matrix correlation issue. The reason for doing so is because correlations can have a significant negative effect on system capacity [27][28][29], and yet the vast majority of relevant SDMA studies, such as those listed in Section 1.2.3, have assumed an uncorrelated channel matrix, even though such correlations are almost guaranteed to occur in practice. A notable exception is the work by Nishimura et al. [18], but that is severely limited in its scope to only two users, with two antennas each.

In this thesis we study the negative effects of correlations and propose and explore decorrelation techniques for mitigating theses effects. Although the primary focus is on the correlation issue, the proposed techniques are based on the issue of antenna and user 
selection, and are also applicable to the power gain imbalance problem, so these two additional issues are also investigated in a secondary fashion.

We propose decorrelation algorithms, in single user and multi-user propagation scenarios, in a MIMO antenna configuration where the basestation antennas are distributed as multi-antenna arrays distributed at different sites in the microcell. The arrays are assumed to be mounted at low elevations on building walls or street poles, and are assumed to communicate with the central base station, where all signal processing is performed, via optical fibers, which are well known to have very low loss and enormous bandwidth, using radio over fiber (RoF) technology [30][31][32][33].

In radio-over-fiber communication systems, the light source (i.e. the laser diode) is modulated (electrical/optical (E/O) converter) by the RF signal, and then the light is transmitted in analog form to the receiving end where it is detected using optical/electrical (O/E) converters (Figure 1.7). So only simple $\mathrm{O} / \mathrm{E}$, and may be $\mathrm{E} / \mathrm{O}$, converters and RF amplifiers are needed at the antenna arrays [30][31][33]. This centralized processing infrastructure is simple because there is no need for RF frequency conversion or sophisticated receiver hardware at the antenna sites and it allows dynamic RF carrier and/or OFDM sub-carrier allocation from the centralized base station.

Having the arrays placed at such low elevation and close to the mobile user (within few hundreds of meters) creates a different scattering environment from one array to another and may create a (LOS) condition for the channel matrix.

With such an antenna configuration, the space domain is significantly improved as different channel properties are created at each array, due to different surrounding scatterers, such as angular spread, angular spectrum, angles of arrival and departure, LOS, shadowing and pathloss. As shown in this thesis, this configuration enhance the balance of the MIMO channel matrix and leads to significant decrease in the eigenvalue spread and the condition number of the channel matrix which results in the reduction of intra-user and inter-user correlations, provided the proposed decorrelation algorithms are used. 


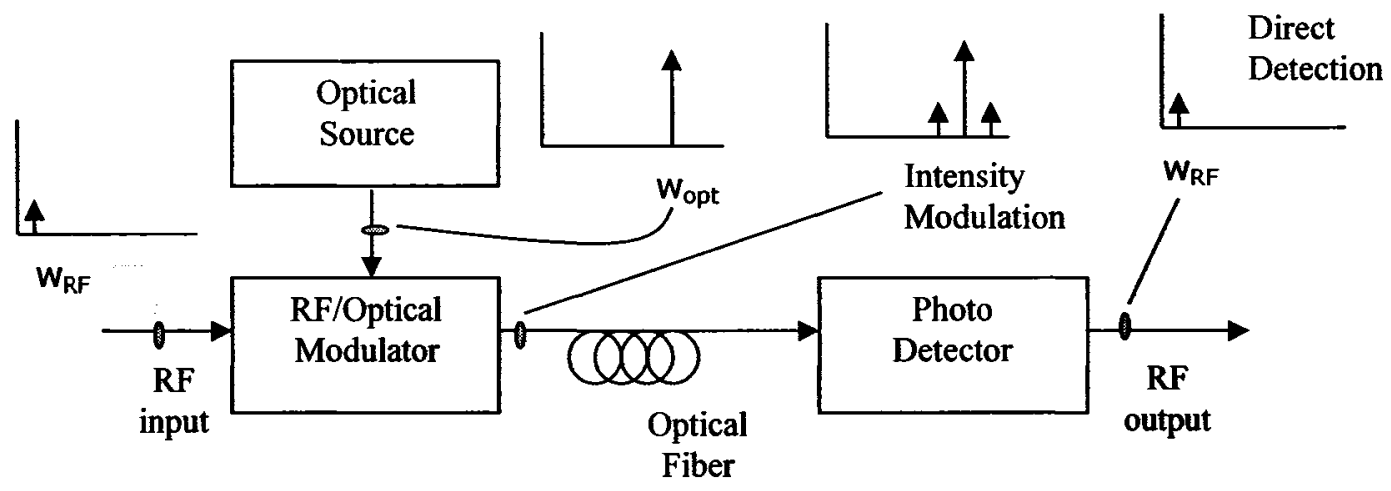

Figure 1.7. Intensity modulation direct detection (IMDD) analog optical link.

In addition we show that the near-far problem is significantly reduced in the distributed base station antennas scenario. While a MIMO channel with co-located base station antennas may cause a severe imbalance within the channel matrix, due to different distances of the mobile users to the base station, the distributed base station antennas configuration significantly balances the channel matrix and more balance is achieved with more distributed arrays.

The main thesis contributions are as follows.

- We model a correlated, frequency selective Ricean fading MIMO channel in which multi-antenna mobile users are communicating with the basestation through multiantenna arrays that are distributed over different sites within the microcell. Each array may have a different pathloss, shadowing and correlation matrix. We generate the correlation matrices assuming that a multi-cluster of waves is impinging on each antenna array and each cluster may have different scattering parameters (angular spread, angular spectrum and angle of arrival /or departure). 
- In a single user propagation scenario; we develop selection algorithms both in the space dimension (distributed antennas) and in the frequency dimension (OFDM tones) that aim to reduce the intra-user correlation and maximize the power transfer gain of the channel matrix which results in significant enhancement in system performance.

- In a downlink multiuser (each with several antennas) propagation scenario, we develop a decorrelation algorithm that enhances the spatial separability of the mobile users. The decorrelation algorithm selects mobile users, in each OFDM tone, with low pair-wise cross correlations. The ability of the base station to spatially separate several users depends on the pair wise cross-correlations between the channel matrices of the users. For a multi-user propagation scenario two types of correlations have to be taken into account; the correlation between the entries of the channel matrix of each user (intra-user correlations), and the correlation between the channel matrix of a user to that of other users (inter-user correlations). To be able to separate users spatially (using SDMA techniques), it is essential that their channel matrices be decorrelated. If it happens that two or more users come close to each other (or the scattering environment is in such a way that correlates the spatial signatures of the users) and the antennas of the basestation are co-located then it's highly likely that their channel matrices be highly correlated. But if the antennas of the basestation are distributed as arrays and only users with low pair-wise cross-correlations are selected in each tone, then the system performance will be significantly enhanced. Distributing the arrays around the users also balances the channel matrix leading to significant reduction in the effect of the near-far problem. We develop a SDMA signaling scheme in which several data symbols can be spatially multiplexed to each selected user over each assigned OFDM tone (water filling is used to determine the relative power of each symbol) with high reliability and with good system throughput.

- The decorrelation algorithm shows significant improved performance in another simpler downlink SDMA zeroforcing signaling scheme, where each selected user has 
a single antenna and the base station is transmitting one symbol to each user in each assigned OFDM tone.

- We propose a decorrelation algorithm for an uplink multi-user propagation scenario. We use an uplink SDMA signaling scheme in which the basestation, with distributedadaptive antenna arrays, employs the decorrelation algorithm to improve the spatial separation of mobile users and reduce the training time needed for the convergence of the filter weights (using an iterative least mean square (LMS) adaptive algorithm). Distributing the base station antennas also contributes to the reduction of the training time as it reduces the eigenvalue spread of the channel matrix.

- In another uplink multi-user propagation scenario, we extend the decorrelation algorithm into a signaling scheme in which mobile users spatially multiplex their signals (Alamouti space-time-block codes) to the distributed antennas base station. The decorrelation algorithm significantly improves the spatial separation of the selected users, in each assigned OFDM tone, leading to significant performance improvement.

These contributions have been presented in the following journal and conference papers:

1. N. H. Dawod, I. Marsland and R. Hafez "Improved transmit null steering for MIMOOFDM downlinks with distributed basestation antenna arrays," IEEE Journal on Selected Areas in Communications, vol. 24, pp. 419-426, March 2006.

2. N. H. Dawod, R. Hafez and I. Marsland "An uplink SDMA system with reduced nearfar problem and inter-user correlations," accepted for publication at IEEE Vehicular Technology Conference -Fall, Montreal, Canada, Sept. 2006.

3. N. H. Dawod, R. Hafez and I. Marsland "A multiuser zeroforcing system with reduced near-far Problem and MIMO channel correlations," IEEE Canadian Conference on Electrical and Computer Engineering, Ottawa Canada, May 2006, pp. 306-309,.

4. N. H. Dawod, R. Hafez and I. Marsland "Multicarrier SDMA system with reduced intra-user cross-correlations," German Space Center, 5th international workshop, multi-carrier spread-spectrum MC-SS 2005, Oberpfaffenhofen, Germany, Sept.2005, pp. 283-290.

5. N. H. Dawod, R. Hafez and I. Marsland "Comparative performance of an enhanced downlink SDMA based MIMO-OFDM system with a TDMA based MIMO-OFDM system," LASTED, CSA 2005, Banff, Canada, July 2005, pp. 32-35. 
6. N. H. Dawod, R. Hafez and I. Marsland "Uplink multiuser MIMO-OFDM in widely spaced antenna arrays," IEEE Canadian Conference on Electrical and Computer Engineering 2005, Saskatoon, Canada, May 2005, pp. 144-148.

7. N. H. Dawod, R. Hafez and I. Marsland, " Performance of widely spaced antenna arrays in correlated-Ricean MIMO-OFDM channels," World Wireless Congress, $W W C^{\prime} 2005$, San Francisco, USA, May 2005.

8. N. H. Dawod, R. Hafez and I. Marsland "Spatially multiplexing multiuser space-time block codes in a MIMO channel with distributed basestation antenna arrays," Wireless2005, Calgary, Canada, July 2005.

9. N. H. Dawod, R. Hafez and I. Marsland, "Performance of MIMO-OFDM in widely spaced antenna arrays," Wireless2004, Calgary, Canada, July 2004, pp. 42-46.

The thesis is organized as follows. In Chapter 2, we describe a MIMO-OFDM channel model with distributed basestation antennas. In Chapter 3, we present a review of the capacity of MIMO channels, spatial diversity and the impact of the channel parameters on the channel capacity. In Chapters 4,5 and 6 we present our main thesis contributions. In Chapter 4, we present antenna selection algorithms that aim at the decorrelation of single-user MIMO channel matrix. Decorrelation of downlink multi-user MIMO channels is presented in Chapter 5, and decorrelation of uplink multi-user MIMO channels is presented in Chapter 6. Our conclusions are presented in Chapter 7, also with suggestions for future research. Finally, a brief review on beamforming and SDMA is given in appendix $\mathrm{A}$. 


\section{Chapter 2}

\section{A MIMO Channel Model with Distributed Basestation Antennas}

Wireless communication systems are continually targeting the high data rates and link reliability of wireline systems. Fading, interference, and the limitation of power, bandwidth and complexity are obstacles in the way of this target. This is true when looking at both systems by the eye of time and frequency domains only, but adding the recently exploited space domain (pioneered by Foschini [3] and Telatar [4]), by employing multiple transmit and receive antennas to the wireless link, greatly improves the wireless channel spectral efficiency [34]. This high spectral efficiency is achievable at no increase in power nor bandwidth of the transmitted signal when the channel is richly scattered and with accurately tracked variations. In this chapter we describe a practical MIMO channel model, in which the basestation antennas are distributed as multi-antenna arrays, that will be considered in the signaling schemes presented in chapters four to six of this thesis.

\subsection{Signal Distortion in Wireless Channels}

The transmitted signal, while propagating to the receiving antenna, will be subjected to reflections and scattering from the physical environment, so the signal will follow multiple spatial paths to reach its destination. The signal will be attenuated due to pathloss, long term fading (shadowing) and short term fading (multipath fading).

\subsubsection{Pathloss}

Caused mainly by inverse distance factor, the pathloss (in $\mathrm{dB}$ ) for a signal path of length $d>d_{0}$ metres where $d_{0}$ is the close-in reference distance, is given by [35][36]

$$
P L=P L_{0}+10 \gamma \log _{10}\left(d / d_{0}\right)
$$

where $P L_{0}$ is the pathloss at a distance of $d_{0}$, and $\gamma$ is the pathloss exponent. 


\subsubsection{Long Term Fading (Shadowing)}

Shadowing is caused by obstacles between the transmitter and the receiver such as buildings, trees, mountains, etc. It is known as long term fading because the effect varies slowly over time. Jakes has observed that the logarithm of the average of the received power is close to normal distribution so its said that shadowing (in $\mathrm{dB}$ ) has a normal distribution, with zero mean and a standard deviation of $\sigma_{s}$, where it depends on the physical environment [37][38][39].

\subsubsection{Short Term Fading (Multipath Fading)}

Short term fading results from receiving different slightly delayed copies of the signal at the receiving antenna. Copies that have almost the same phase will add constructively and copies with different phases will add destructively. Different phases are due to different travelled distances and/or different scattering environments. This phenomenon causes large variations in signal power even when the mobile device is moved over only a short distance. For example at a carrier frequency of $2.4 \mathrm{GHz}$ the opposite phase case occurs at a movement of $\lambda_{c} / 2=6.25 \mathrm{~cm}$, so significant power variations occur frequently.

According to the central limit theorem, the aggregation of a large number of independent identically distributed copies of the signal (that result in fading) will make the received signal (the in-phase and quadrature phase components) appear to be an independent zero mean Gaussian process whose envelope is Rayleigh distributed.

This multipath propagation spreads the signal in the frequency domain due to variation of the channel during signal transmission (time-selective fading), spreads the signal in the time domain due to delayed arrival of the signal copies (frequency-selective fading), and spreads the signal in the angular domain because the signal is received randomly from different directions depending on the environment (space-selective fading). 


\subsubsection{Time Selective Fading}

The fading process affecting the received signal varies randomly over time, resulting in time-selective fading. These variations are caused by slight movements of the scatterers (e.g., due to wind), the transmitter, and/or the receiver. The speed by which the channel varies is a function of the speed of movement of the objects and of the RF frequency used by the signal. The coherence time, is used to characterize the time selectivity of the channel. It is a measure of how fast the channel changes in time. The larger the coherence time the slower the channel fluctuates during signal transmission.

\subsubsection{Frequency Selective Fading}

In a multipath propagation environment, each path will introduce a different propagation delay, depending on the length of the path. The separation between the paths increases exponentially with the delay, so it is normally not fixed, and path amplitudes decreases exponentially with the delay. The range of possible delays is a random variable whose variability is due to channel variations, and its root mean square (rms) value is called the delay spread [40]. The delay spread makes the channel act like a multi-tap finite impulse response (FIR) filter which causes the fading of the channel to be frequency selective.The coherence bandwidth, characterizes the frequency selectivity of the channel and is inversely proportional to the delay spread [40].

\subsubsection{Space Selective Fading}

Scatterers, surrounding the receiving antenna, act like virtual transmitting antennas that transmit the same signal but scaled differently and delivered from different angles to the receiving antenna. The range of angles of arrival (AoA) of waves to the receiving antenna is called the angular spread. This angular spread is a random variable with pdf $p_{\theta}(\theta)$, the angular spectrum, which depends on the distribution of scatterers around the receiving array [41]. Because the amplitude of the signal induced on the receiving antenna changes according to the received (differently scaled) waves of different AoA, 
space-selective fading is the natural result of this angular spread. The coherence distance characterizes the space-selective fading and is inversely proportional to the angular spread. The autocorrelation between the signals induced on an antenna array due to the arrival of a wave with AoA of $\theta_{1}$ and another wave with AoA of $\theta_{2}$ determines the coherence distance [41]. The angular spread at the transmitting antenna is the range of angles of departure (AoD) of the waves that scatter at the scatterers, in the vicinity of the transmitter, that deliver these waves to the receiver.

\subsection{Physical Modeling of MIMO Channels}

As shown in Figure 2.1, assume we have a linear antenna array composed of $n_{R}=2$ antenna elements spaced uniformly by a distance $D_{R}$ and that a signal $s(t)$ is impinging on this array with an angle of arrival of $\theta_{R}$, with

$$
s(t)=v(t) e^{j 2 \pi f_{c} t}
$$

where $v(t)$ is the lowpass information signal of bandwidth $W$, and $f_{c}$ is the carrier frequency.

This wave will induce a signal on each antenna element of the array. After impinging on the first antenna element, the wavefront will travel distance $D_{R} \cos \left(\theta_{R}\right)$ and then impinge on the second antenna element after $T_{\theta}$ seconds,

where

$$
T_{\theta}=\frac{D_{R} \cos \left(\theta_{R}\right)}{c}=\frac{D_{R} \cos \left(\theta_{R}\right)}{\lambda_{c} f_{c}}
$$

and $c$ is the speed of light and $\lambda_{c}=c / f_{c}$ is the wavelength of the carrier.

Assume that the signal induced on the first element is $r_{1}(t)=s(t)$. Although the power pattern of the first antenna element is not identical to that of the second one and the wave 


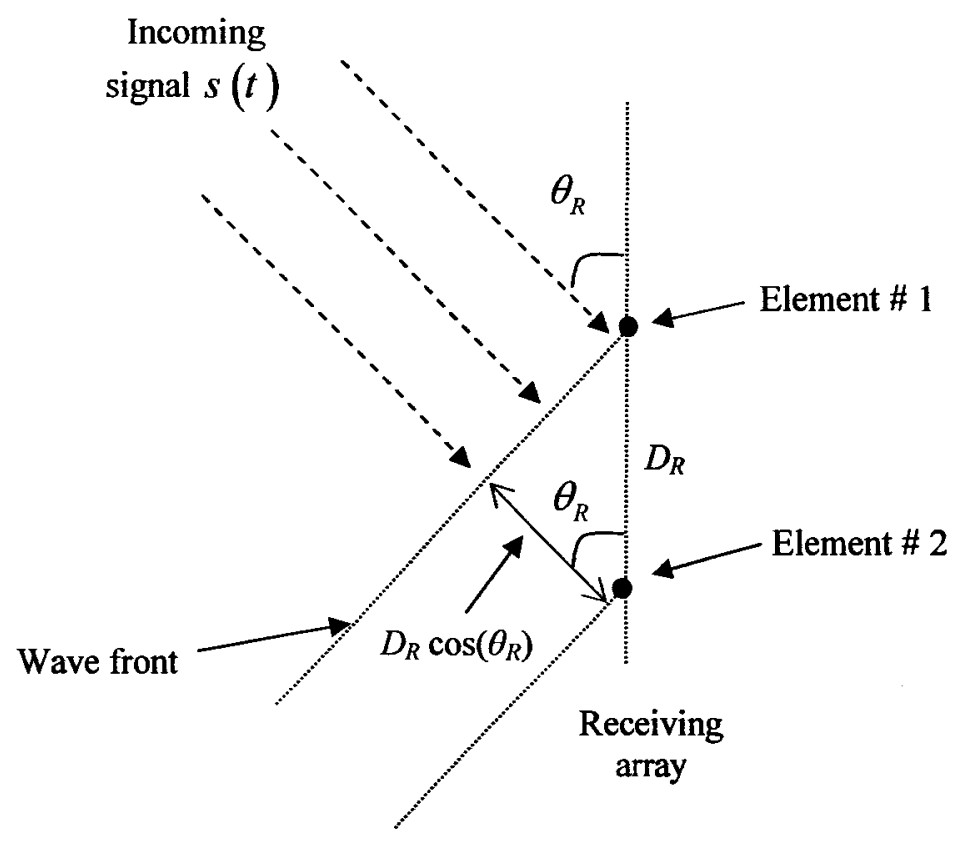

Figure 2.1. Wave front impinging on a two element antenna array.

while propagating the small distance $D_{R} \cos \left(\theta_{R}\right)$ may be attenuated, we can nonetheless assume that the additional attenuation is negligible, so the signal induced on the second element will be

$$
r_{2}(t) \cong s\left(t-T_{\theta_{R}}\right)=v\left(t-T_{\theta_{R}}\right) e^{j 2 \pi f_{c}\left(t-T_{\theta_{R}}\right)} .
$$

Furthermore, under the assumption that the additional propagation delay is much less than the transmission symbol rate (i.e., under narrow band conditions such that $\left.T_{\theta_{R}} \ll 1 / W\right)$, then

$$
v\left(t-T_{\theta_{R}}\right) \cong v(t),
$$




$$
\begin{aligned}
r_{2}(t) & \cong v\left(t-T_{\theta_{R}}\right) e^{j 2 \pi f_{c}\left(t-T_{\theta_{R}}\right)} \cong v(t) e^{j 2 \pi f_{c} t} e^{-j 2 \pi \frac{D_{R} \cos \left(\theta_{R}\right)}{\lambda_{c}}} \\
& \cong s(t) e^{-j 2 \pi \frac{D_{R} \cos \left(\theta_{R}\right)}{\lambda_{c}}}
\end{aligned}
$$

We conclude from (2.6) that the signals induced on the antennas essentially differ by only a phase shift that is a function of the angle of arrival, carrier frequency, and antenna separation.

\subsubsection{Line-of-sight SIMO Channel}

We consider a LOS single-input multi-output (SIMO) channel in free space environment (i.e. without any reflectors or scatterers) and only a direct signal path between each antenna pair (Figure 2.2). We also assume that the dimension of the antenna array is much smaller than distance between the transmitter and the receiver. The continuous-time impulse response between the transmit antenna and $i^{\text {th }}$ receive antenna is given by:

$$
h_{i}(\tau)=a \delta\left(\tau-d_{i} / c\right) \quad i=1, \cdots, n_{R}
$$

where $d_{i}$ is the distance between the transmit antenna and $i^{\text {th }}$ receive antenna, $c$ is the speed of light and $a$ is the attenuation of the path, which is assumed to be the same for all antenna pairs. Assuming $W \ll 1 /\left(d_{i} / c\right)$ where $W$ is the transmission bandwidth, the baseband channel gain is given by

$$
g_{i}=a \exp \left(-\frac{j 2 \pi f_{c} d_{i}}{c}\right)=a \exp \left(-\frac{j 2 \pi d_{i}}{\lambda_{c}}\right)
$$

and the SIMO signal model can be written as

$$
\mathbf{r}=\mathbf{g}_{\mathrm{SIMO}} s+\mathbf{w}
$$

where $s$ is the transmitted symbol, $\mathbf{w}$ is the noise and $\mathbf{r}$ is the received vector. We may assume the paths between the transmit antenna and all of the receive antennas to be parallel, as we assumed the dimension of the antenna array is much smaller than distance between the transmitter and the receiver, so 


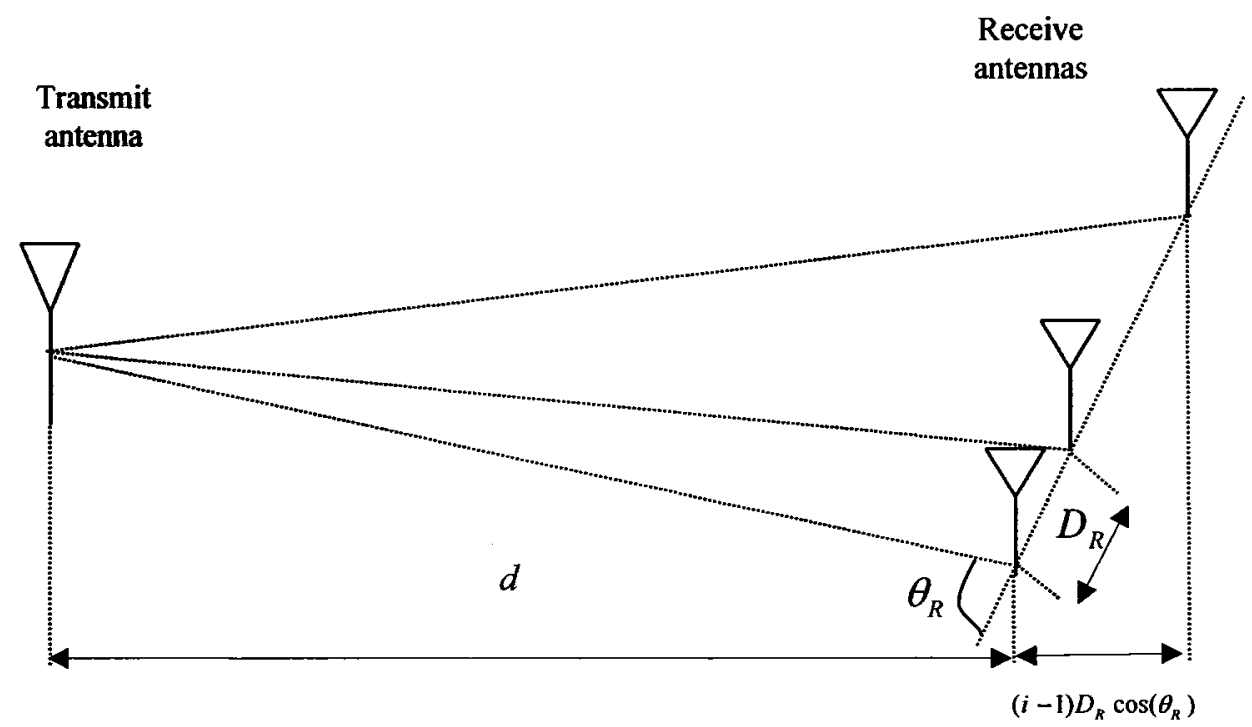

Figure 2.2. Line of sight SIMO channel.

$$
d_{i} \cong d+(i-1) D_{R} \cos \left(\theta_{R}\right) \quad i=1, \cdots, n_{R}
$$

where $d$ is the distance from the transmit antenna to the first receive antenna and $\theta_{R}$ is the angle of arrival (AoA). The term $(i-1) D_{R} \cos \left(\theta_{R}\right)$ is the displacement of receive antenna $i$ from receive antenna 1 in the direction of the line of sight. The spatial signature $\mathbf{g}_{\mathrm{SIMO}}$ can be written as [41]

$$
\begin{aligned}
\mathbf{g}_{\mathrm{SIMO}} & =\boldsymbol{a} \exp \left(-\frac{j 2 \pi d}{\lambda_{c}}\right)\left[\begin{array}{c}
1 \\
\exp \left\{-j 2 \pi \Delta_{R} \cos \left(\theta_{R}\right)\right\} \\
\exp \left\{-j 2 \pi 2 \Delta_{R} \cos \left(\theta_{R}\right)\right\} \\
\vdots \\
\exp \left\{-j 2 \pi\left(n_{R}-1\right) \Delta_{R} \cos \left(\theta_{R}\right)\right\}
\end{array}\right] \\
& =a \sqrt{n_{R}} \exp \left(-\frac{j 2 \pi d}{\lambda_{c}}\right) \mathbf{e}_{\mathbf{R}}\left(\theta_{R}\right)
\end{aligned}
$$


where $\Delta_{R}=D_{R} / \lambda_{c}$ is the receive antenna separation normalized to the carrier wavelength and $\mathbf{e}_{R}\left(\theta_{R}\right)$ is the unit spatial signature, given by

$$
\mathbf{e}_{R}\left(\theta_{R}\right)=\frac{1}{\sqrt{n_{R}}}\left[\begin{array}{c}
1 \\
\exp \left\{-j 2 \pi \Delta_{R} \cos \left(\theta_{R}\right)\right\} \\
\exp \left\{-j 2 \pi 2 \Delta_{R} \cos \left(\theta_{R}\right)\right\} \\
\vdots \\
\exp \left\{-j 2 \pi\left(n_{R}-1\right) \Delta_{R} \cos \left(\theta_{R}\right)\right\}
\end{array}\right]
$$

Since $\mathbf{g}_{\text {SIMO }}$ is a rank one channel with only one spatial subchannel, with a gain of $a \sqrt{n_{R}}$, through which only one symbol can be transmitted, so this antenna configuration does not provide spatial multiplexing gain, it only provides array gain. The optimal receiver adjusts for the different delays so that the received signals at the antennas can be combined constructively, yielding a $n_{R}$ fold array gain but no diversity no spatial multiplexing gain.

\subsubsection{Line of Sight MISO Channel}

The multi-input single-output (MISO) channel has multiple transmit antennas and single receive antenna. Similar to the SIMO channel case, the signal model is given by

$$
\begin{aligned}
& r=\mathbf{g}_{\mathrm{MISO}}^{H} \mathbf{s}+w, \\
& \mathbf{g}_{\mathrm{MISO}}=a \sqrt{n_{T}} \exp \left(-\frac{j 2 \pi d}{\lambda_{c}}\right) \mathbf{e}_{T}\left(\theta_{T}\right),
\end{aligned}
$$

where $\Delta_{T}=D_{T} / \lambda_{c}$ is the transmit antenna separation normalized to the carrier wavelength, $\theta_{T}$ is the angle of departure and $\mathbf{e}_{T}\left(\theta_{T}\right)$ is the transmit unit spatial signature, given by 


$$
\mathbf{e}_{T}\left(\theta_{T}\right)=\frac{1}{\sqrt{n_{T}}}\left[\begin{array}{c}
1 \\
\exp \left\{-j 2 \pi \Delta_{T} \cos \left(\theta_{T}\right)\right\} \\
\exp \left\{-j 2 \pi 2 \Delta_{T} \cos \left(\theta_{T}\right)\right\} \\
\vdots \\
\exp \left\{-j 2 \pi\left(n_{T}-1\right) \Delta_{T} \cos \left(\theta_{T}\right)\right\}
\end{array}\right]
$$

This antenna configuration also has only one spatial subchannel. There is no diversity or spatial multiplexing gain. The phase of the signal transmitted from each antenna is adjusted so that they add constructively at the receiving antenna, yielding $n_{T}$ fold array gain (transmit maximum ratio combining).

With multiple transmit antennas, the transmitter may employ SDMA by using beamforming to simultaneously serve multiple users at different locations. A brief review on beamforming and SDMA techniques can be found in Appendix A.

\subsubsection{Line of Sight MIMO Channel}

We consider now the MIMO propagation scenario where an array of antennas is transmitting to an array of receive antennas with only direct line of sight between the arrays as shown in Figure 2.3. The channel gain between $k^{\text {th }}$ transmit antenna and the $i^{\text {th }}$ receive antenna is

$$
g_{i k}=a \exp \left(-j 2 \pi d_{i k} / \lambda_{c}\right)
$$

where $d_{i k}$ is the distance between the $k^{\text {th }}$ transmit antenna and the $i^{\text {th }}$ receive antenna. Assuming again the array dimensions are much smaller than the array separation then

$$
d_{i k}=d+(i-1) \Delta_{R} \lambda_{c} \cos \left(\theta_{R}\right)-(k-1) \Delta_{T} \lambda_{c} \cos \left(\theta_{T}\right)
$$

where $d$ is the distance between the transmit antenna 1 and the receive antenna one. The channel matrix can be written as

$$
\mathbf{G}_{L O S}=a \sqrt{n_{R} n_{T}} \exp \left(-\frac{j 2 \pi d}{\lambda_{c}}\right) \mathbf{e}_{R}\left(\theta_{R}\right) \mathbf{e}_{T}^{H}\left(\theta_{T}\right)
$$




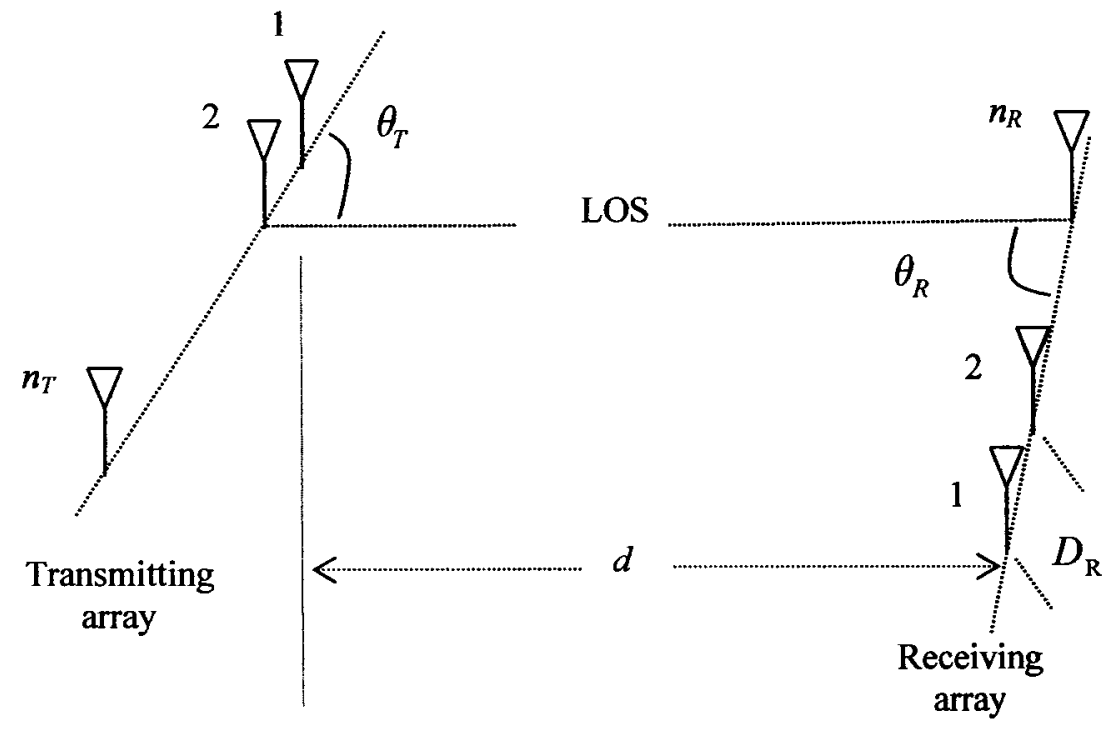

Figure 2.3. Line of sight MIMO channel.

Despite having multiple transmit antennas and multiple receive antennas, the line of sight condition makes the rank of $\mathbf{G}$ equal one with a unique non-zero singular value of $a \sqrt{n_{R} n_{T}}$. Again no spatial multiplexing gain or diversity gain is realized. The optimum signaling scheme is to make the transmitted signals add constructively in phase at each receive antenna (i.e. transmit $\mathrm{MRC}$ ) and then the signals at each receive antenna are further constructively combined (i.e. receive $\mathrm{MRC}$ ).

In a line of sight environment, whether SIMO, MISO or MIMO, the channel provides array gain only but no spatial multiplexing gain.

\subsubsection{Non-LOS Scattering MIMO Channel}

In a multipath propagation communication environment, the transmitted signal will follow numerous paths to the receiver, in addition to (or instead of) a direct line-of sight path. In a MIMO channel, the antenna configurations and the nature of the scattering environment around the antennas at both sides of the MIMO channel characterize the 
spatial correlation properties within the channel matrix. If the transmitting and receiving arrays are located in a rich scattering environment and are with well-designed geometry, then the channel matrix entries can be modeled as a white channel of independent zero mean circularly symmetric complex Gaussian random variables [28].

Such a rich scattering environment, in which the transmitted signals received uniformly from all directions around the receiver's array, is not an appropriate model for all situations. A more realistic (and generalized) model is to assume the existence of clusters (or clouds) of scatterers surrounding the basestation antenna arrays and the existence of other clusters of scatterers surrounding the user antenna array, as shown in Figure 2.4, for example. These clouds account for the spatial correlation properties observed from the transmit and receive antenna arrays. We also assume that the correlation among the receiving elements is independent of the transmitter and the correlation properties at the transmitter do not depend on the receiver.

Based on these assumptions, we use a correlated stochastic channel model, with channel matrix [23][24][43][28][34]

$$
\mathbf{G}_{N L O S}=\mathbf{R}_{R}^{1 / 2} \mathbf{G}_{W} \mathbf{R}_{T}^{1 / 2}
$$

where the entries of $\mathbf{G}_{W}$ are zero-mean unit-variance independent complex Gaussian random variables, and $\mathbf{R}_{R}$ and $\mathbf{R}_{T}$ are $n_{R} \times n_{R}$ and $n_{T} \times n_{T}$ spatial correlation matrices, at the receiver and transmitter sides, respectively. These matrices capture the correlations within the MIMO channel that depend on the scattering environment parameters such as the angular spread, the angular spectrum, angles of arrival and departure, and antenna configurations. Their elements are given by [44][45][46]

$$
\begin{aligned}
& {\left[\mathbf{R}_{R}\right]_{n m}=\mathrm{E}\left[\exp \left\{-j 2 \pi(n-m) \Delta_{R} \cos \theta_{R}\right\}\right]} \\
& \quad=\int_{\alpha / 2}^{\alpha / 2} \exp \left\{-j 2 \pi(n-m) \Delta_{R} \cos \theta_{R}\right\} P_{R}\left(\theta_{R}\right) d \theta_{R}
\end{aligned}
$$

and 


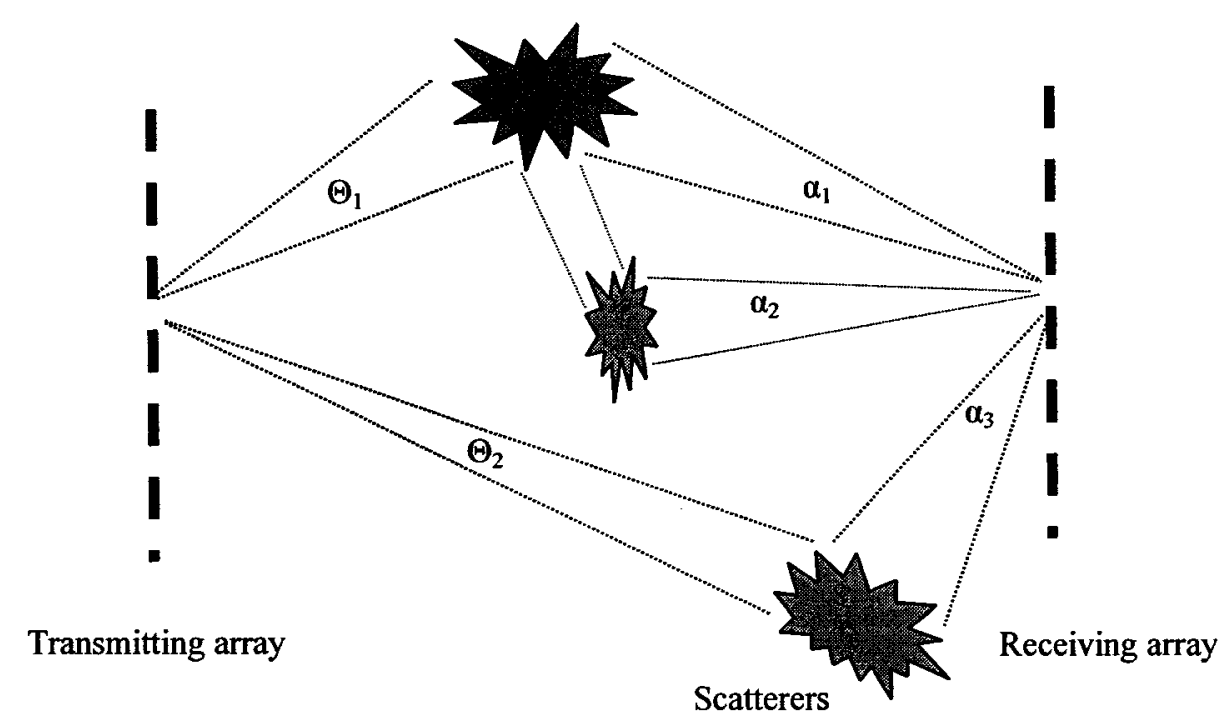

Figure 2.4. Two transmit and three receive clusters of waves.

$$
\left[\mathbf{R}_{T}\right]_{n m}=\int_{\alpha / 2}^{\alpha / 2} \exp \left\{-j 2 \pi(n-m) \Delta_{T} \cos \theta_{T}\right\} P_{T}\left(\theta_{T}\right) d \theta_{T}
$$

where $\Delta_{R}$ and $\Delta_{T}$ are the normalized antenna separations of the receive and transmit arrays, and $P_{R}\left(\theta_{R}\right)$ and $P_{T}\left(\theta_{T}\right)$ are the combined power angle profiles of the scatters in the vicinity of the receiver and transmitter, respectively, and $\alpha$ is the angular spread.

The group of waves arriving from a single cluster of scatters will arrive from a range of angles constrained by the angular spread of the cluster, centered about the central AoA of the cluster. The strength of the waves will depend on the angle of arrival relative to the central AoA, with maximum strength observed in the direction of the center of the cluster (the central AoA). This power angle profile of the waves from the cluster depends on the locations, amplitudes, densities and nature of the scatterers in the cluster. If the scatterers are, almost, identical and evenly distributed around the cluster, then the power angle profile becomes, almost, uniform (when properly normalized) around the central AoA (with a certain angular spread) [48]. Pedersen et al. [49] and Adachi et al. [50] showed 
that the normalized power angle profile might also be distributed as truncated Laplacian or truncated Gaussian, respectively. The angle of arrival, angle of departure and the angular spread are in general time-variant due to the movement of the mobile station and the changing of scatterers around the antennas, however they are assumed to remain fixed for the duration of the transmission of a block of data [44].

The combined power angle profiles, $P_{R}\left(\theta_{R}\right)$ and $P_{T}\left(\theta_{T}\right)$, are the normalized sums of the power angle profiles of all the clusters in the vicinities of the receiver and the transmitter, respectively. The power angle profile differs from the array radiation pattern. The radiation pattern is determined by the design of the antenna elements (e.g., dipole, elliptical, helical, etc.), directors and reflectors. All of theses factors shape the power pattern and set the gain of the antenna array in all directions (angles). The power angle profile on the other hand, is mainly shaped by the channel environment.

\subsection{A MIMO Channel Model with Distributed Basestation Antennas}

We now consider the realistic multi-point to multi-point channel model that we will use throughout this thesis. In this model, as shown in Figure 2.5, there are $n_{P}$ antenna arrays distributed around a microcell, each with $n_{L}$ antennas elements and each mobile station (user) has $n_{R}$ antennas. The arrays are placed at low heights $(5-20$ metres) above the ground (for example on street poles or building walls), and are connected via optical fiber to a central base station, using radio over fiber (RoF) technology, where all signal processing is performed.

The MIMO system model, seen by each user, is now composed of $n_{P} n_{L}$ antennas at the base station and $n_{R}$ antennas at each user. The fading of the channel between the antennas of any of the arrays and the mobile station antennas is assumed to be frequency selective, with both line-of-sight and scattered components. The channel is assumed quasi-static and the channel state information (CSI) is assumed to be known at the basestation (for example, by using an uplink to return channel measurements collected during the transmission of downlink training sequences). From site to site, the average power, the Ricean $K$-factor and the correlation (between antenna elements) differ due to 


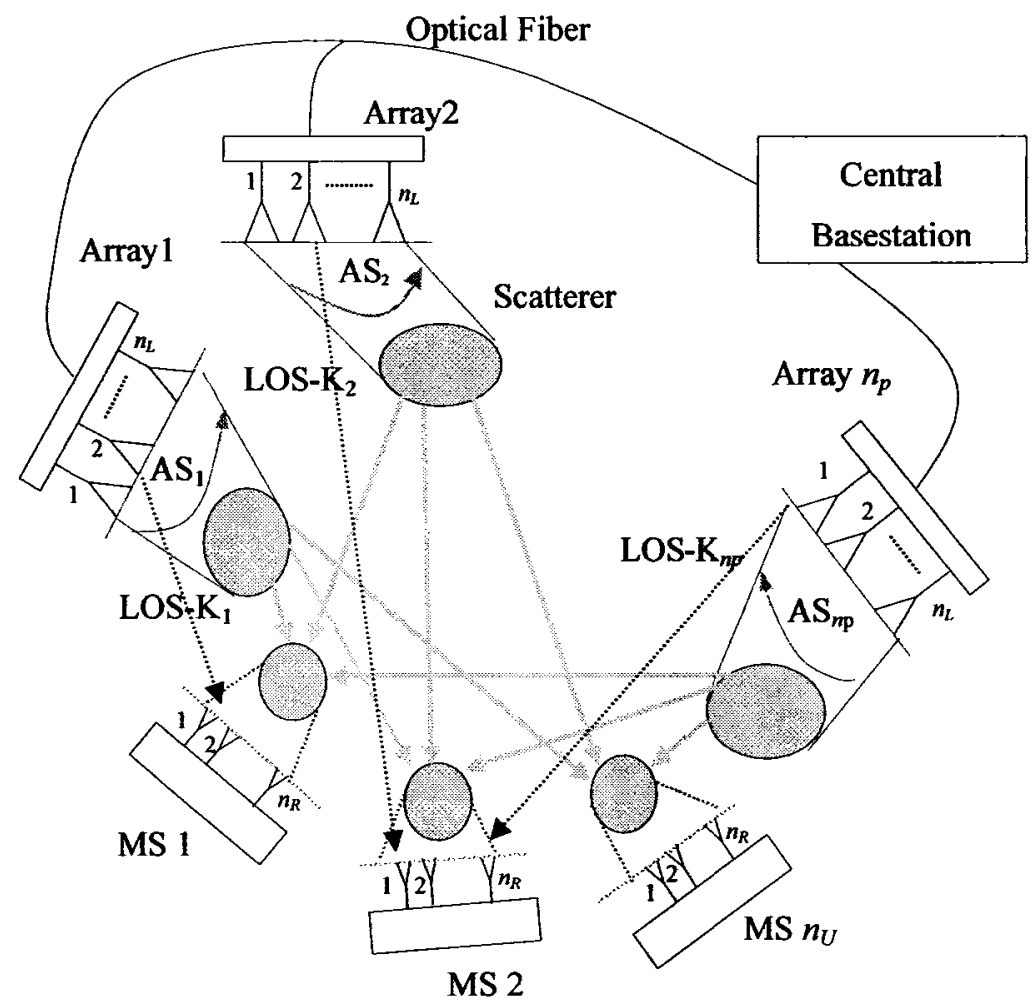

Figure 2.5. Multi-users in a correlated Ricean channel with distributed basestation antennas.

different path losses, shadowing and scattering environments around the antennas. The shadowing component is assumed to have a lognormal distribution. The correlation properties between the antennas within each array depend on the angular spread, angular spectrum and direction of arrival or departure of the impinging wave and on the arrangement of the antennas and the spacing between them.

We represent the frequency selective fading channel by a tapped delay line with $L$ taps. Each tap is represented by the above mentioned channel model which takes into account the power delay profile, path loss, and the $K$-factor (for each array). The $l^{\text {th }}$ tap of a user's $n_{R} \times n_{P} n_{L}$ time domain channel matrix (assuming downlink transmission) is represented as 


$$
\mathbf{G}_{l}=\left[\mathbf{G}_{l, 1} \mathbf{G}_{l, 2} \ldots \mathbf{G}_{l, n_{P}}\right]
$$

where $\mathbf{G}_{l, p}$ is the $n_{R} \times n_{L}$ channel matrix between the $p^{\text {th }}$ array and the user antennas, with

$$
\mathbf{G}_{l, p}= \begin{cases}\mathrm{PDP}_{l} \mathrm{PL}_{p}\left(\sqrt{\frac{K_{p}}{K_{p}+1}} \beta_{\mathrm{LOS}, p} \mathbf{G}_{\mathrm{LOS}, p}+\sqrt{\frac{1}{K_{p}+1}} \beta_{\mathrm{NLOS}, l, p} \mathbf{G}_{\mathrm{NLOS}, l, p}\right) & \text { if } l=0 \\ \mathrm{PDP}_{l} \mathrm{PL}_{p} \beta_{\mathrm{NLOS}, l, p} \mathbf{G}_{\mathrm{NLOS}, l, p} & \text { if } l \neq 0\end{cases}
$$

where $\mathrm{PDP}_{l}$ is the gain of the $l^{\text {th }}$ tap of the normalized power delay profile, $\mathrm{PL}_{p}$ is the path loss for the signals from the $p^{\text {th }}$ array to the user, $K_{p}$ is the Ricean $K$-factor for the $p^{\text {th }}$ array, $\beta_{\mathrm{LOS}, p}$ and $\beta_{\mathrm{NLOS}, l, p}$ are lognormal random variables that represent the shadowing for the LOS and the scattering components of the channel, respectively, and $\mathbf{G}_{\mathrm{LOS}, p}$ and $\mathbf{G}_{\mathrm{NLOS}, l, p}$ are the $n_{R} \times n_{L}$ channel matrices between the $p^{\text {th }}$ array and the user for the LOS and the scattering components of the channel, respectively. Each of the $\mathbf{G}_{l, p}$ matrices will have different $K$-factors, path losses and shadowing. The path loss $\left(\mathrm{PL}_{p}\right)$ depends on the distance between the user and the $p^{\text {th }}$ array. The matrix $\mathbf{G}_{\mathrm{Los}, p}$ in (2.23) is modeled according to (2.18) as

$$
\mathbf{G}_{\mathrm{LOS}, p}=a \sqrt{n_{R} n_{T}} \exp \left\{-\frac{j 2 \pi d_{p}}{\lambda}\right\} \mathbf{e}_{\mathrm{R}}\left(\theta_{\mathrm{R}, p}\right) \mathbf{e}_{\mathrm{T}}\left(\theta_{\mathrm{T}, p}\right)^{H}
$$

where $\lambda_{c}$ is the wavelength of the carrier, $d_{p}$ is the distance between transmit antenna 1 of the $p^{\text {th }}$ array and receive antenna 1 (see Figure 2.3), $\theta_{\mathrm{R}, p}$ is the angle of incidence of the LOS component onto the receive antenna array (the direction of arrival) and $\theta_{T, p}$ is the angle of incidence of the LOS component from the $p^{\text {th }}$ transmit antenna array (the direction of departure). $\mathbf{e}_{\mathrm{R}}\left(\theta_{R, p}\right)$ and $\mathbf{e}_{\mathrm{T}}\left(\theta_{T, p}\right)$ are the unit spatial signatures (representing the differences in the phases of the induced signals on the different antennas) at the receive and transmit arrays, respectively. These are given by (2.12) and (2.15), respectively. 
As described in Section 2.2.4, we use a correlation-based stochastic channel model for the matrix $\mathbf{G}_{\mathrm{NLOS,l}}$, in (2.23), as [23] [24][43][28] [34]

$$
\mathbf{G}_{\mathrm{NLOS}, l, p}=\mathbf{R}_{\mathrm{R}, l, p}^{1 / 2} \mathbf{G}_{\mathrm{W}, l, p} \mathbf{R}_{\mathrm{T}, l, p}^{1 / 2}
$$

where $\mathbf{R}_{R, l, p}$ and $\mathbf{R}_{T, l, p}$ are the $n_{R} \times n_{R}$ and the $n_{T} \times n_{T}$ spatial correlation matrices of the $l^{\text {th }}$ tap, at the user's side and at the basestation side respectively, with entries given by (2.20) and (2.21), respectively.

\subsection{Space-Time OFDM}

The main idea behind OFDM is that since low-rate modulations (i.e. modulations with relatively long duration symbols compared to the channel time characteristics) are less sensitive to multipath interference, it should be better to send a number of low rate streams in parallel than sending one high rate waveform. OFDM divides the frequency spectrum in sub-bands (tones) small enough so that the channel effects are constant (flat) over each given tone. Then a "classical" IQ modulation (BPSK, QPSK, M-QAM, etc) is sent over each tone. Classical signal processing, such as channel coding, power allocation, and adaptive modulation and coding can be applied for a given tone or over all the tones.

The OFDM signal is composed of the sum of $n_{C}$ orthogonal subcarriers where each might be modulated by different PSK or QAM signals. By this orthogonality, inter-carrier interference (ICI) is avoided. To eliminate the inter-symbol interference (ISI) of multipath channels, a guard time of duration larger than the expected delay spread of the channel is included with the OFDM symbol. To maintain orthogonality among the subcarriers, the guard time is formed by extending all the subcarriers into the guard time period. OFDM modulation and demodulation are typically implemented using digital filter banks using the inverse discrete Fourier transform (IDFT) and discrete Fourier transform (DFT).

OFDM has been standardized in several wireless systems, such as digital audio and video broadcasting (DAB, DVB), ADSL, European HIPERLAN/2, Japanese MMAC and 
American wireless LAN such as IEEE 802.11a, IEEE 802.11g. Combining OFDM with MIMO greatly increases wireless link reliability through exploiting diversity and facilitating spatial multiplexing in both frequency and space domains [51][52].

Figure 2.6 shows a block diagram of a baseband OFDM link. The signal $\mathbf{s}=\left[s_{0} s_{1} \cdots s_{n_{C}-1}\right]^{r}$ consists of $n_{C}$ symbols drawn from a constellation with unit average energy. This signal is converted into the time domain using an inverse DFT [53][54], so

$$
\mathbf{z}=\mathbf{F}^{H} \mathbf{s}
$$

where $\mathbf{F}$ is the $n_{C}$-point DFT matrix, with elements

$$
\mathbf{F}_{p q}=\frac{1}{\sqrt{n_{C}}} e^{-j 2 \pi \frac{(p-1)(q-1)}{n_{C}}}
$$

After appending a cyclic prefix of length $L-1$, the transmitted components of the OFDM symbol corresponding to $\mathbf{s}$ are given by

$$
\mathbf{x}=\left[z_{n_{C}-L+1} \cdots z_{n_{C}-2} z_{n_{C}-1} z_{0} z_{1} \cdots z_{n_{C}-1}\right]
$$

Note that the cyclic prefix is merely the last $L-1$ elements of $\mathbf{z}$ repeated prior to $\mathbf{z}$.

During transmission, the elements of the OFDM symbol will be convolved with the $L$-tap channel and then at the receiver the cyclic prefix will be discarded. The $L$ tap impulse response of frequency selective single input single output (SISO) wireless channel is represented by

$$
\mathbf{g}=\left[g_{0} g_{1} \cdots g_{L-1}\right]^{T}
$$

This $\mathbf{g}$ representation is assumed to contain the effects of pulse shaping at the transmitter and the matched filter at the receiver as well as the intervening channel. The resulting vector $\hat{\mathbf{z}}$ of received samples is given by

$$
\hat{\mathbf{z}}=\tilde{\mathbf{G}} \mathbf{x}+\tilde{\mathbf{n}}
$$

where the $n_{C} \times\left(n_{C}+L-1\right)$ channel matrix $\tilde{\mathbf{G}}$ is given by [55] 


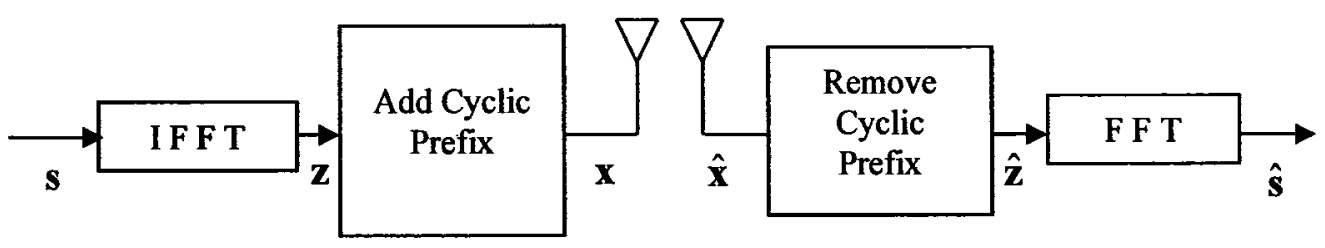

Figure 2.6. Baseband OFDM link.

$$
\tilde{\mathbf{G}}=\left[\begin{array}{cccccccc}
g_{L-1} & \cdots & g_{1} & g_{0} & 0 & 0 & \ldots & 0 \\
0 & g_{L-1} & \cdots & g_{1} & g_{0} & 0 & \ldots & 0 \\
\vdots & 0 & \ddots & \ddots & \ddots & \ddots & \ddots & \vdots \\
0 & \vdots & 0 & g_{L-1} & \ldots & g_{1} & g_{0} & 0 \\
0 & 0 & 0 & 0 & g_{L-1} & \cdots & g_{1} & g_{0}
\end{array}\right]
$$

and $\hat{\mathbf{n}}$ is a vector of independent complex AWGN samples, and with zero mean and variance $\sigma_{n}^{2}$. Because of the cyclic prefix, the linear convolution of the signal with the channel taps can also be expressed as a circular convolution, using

$$
\hat{\mathbf{z}}=\mathbf{G} \mathbf{z}+\tilde{\mathbf{n}}
$$

where the $\mathbf{G}$ is the $n_{C} \times n_{C}$ circulant matrix given by [55] 


$$
\mathbf{G}=\left[\begin{array}{cccccccc}
g_{0} & 0 & \ldots & 0 & 0 & g_{L-1} & \ldots & g_{1} \\
g_{1} & g_{0} & 0 & \ldots & 0 & 0 & \ddots & \vdots \\
\vdots & g_{1} & g_{0} & 0 & 0 & \ddots & 0 & g_{L-1} \\
g_{L-1} & \vdots & g_{1} & \ddots & 0 & \ddots & 0 & 0 \\
0 & g_{L-1} & \vdots & \ddots & g_{0} & \ddots & \ddots & 0 \\
\vdots & 0 & g_{L-1} & \ddots & g_{1} & g_{0} & 0 & 0 \\
\vdots & \vdots & 0 & \ddots & \ddots & \ddots & \ddots & 0 \\
0 & 0 & \ldots & 0 & g_{L-1} & \ldots & g_{1} & g_{0}
\end{array}\right] .
$$

After taking the DFT of $\hat{\mathbf{z}}$, estimates of the original transmitted data vector, $\mathbf{s}$, can be obtained as

$$
\begin{aligned}
\hat{\mathbf{s}} & =\mathbf{F} \hat{\mathbf{z}} \\
& =\mathbf{F G z}+\mathbf{F} \tilde{\mathbf{n}} \\
& =\mathbf{F G} \mathbf{F}^{H} \mathbf{s}+\mathbf{F} \tilde{\mathbf{n}} \\
& =\mathbf{\Lambda} \mathbf{s}+\mathbf{n}
\end{aligned}
$$

where $\mathbf{n}=\mathbf{F} \tilde{\mathbf{n}}$ is another AWGN vector, with the same statistics as $\tilde{\mathbf{n}}$, and $\boldsymbol{\Lambda}=\mathbf{F G F}^{H}$ is a diagonal matrix containing the channel gains experienced by the OFDM tones, it is given by

$$
\Lambda=\operatorname{diag}\left(h_{0} h_{1} \cdots h_{n_{C}-1}\right)
$$

where $h_{k}$ is the channel gain seen by the $k^{\text {th }}$ tone and is given by the DFT of the channel impulse response as

$$
h_{k}=\sum_{l=0}^{L-1} g_{l} e^{-j 2 \pi \frac{k l}{n_{C}}} \quad k=01 \cdots n_{C}-1
$$

We see that the use of OFDM orthogonalizes the delay spread of the channel by converting the frequency selective channel into $n_{C}$ frequency flat channels.

In the case of a frequency selective fading MIMO channel composed of $n_{T}$ transmit and $n_{R}$ receive antennas, each tap of the channel is represented by a $n_{R} x n_{T}$ channel 
matrix $\mathbf{G}_{l}$, with elements $g_{l, j}=\left[\mathbf{G}_{l}\right]_{i j}$ being the channel gain of the $l^{t h}$ tap between the $i^{\text {th }}$ transmit and $j^{\text {th }}$ receive antennas.

As in the single-antenna case, with the employment of an OFDM signal through a MIMO channel, the inverse DFT operation, cyclic prefix addition, convolution with the MIMO channel, cyclic prefix removal and the DFT operation will convert the frequency selective MIMO channel into $n_{C}$ frequency flat MIMO channels. Each tone of the OFDM symbol will see a flat fading MIMO channel composed of $n_{T}$ transmit antennas and $n_{R}$ receive antennas as given by

$$
\mathbf{y}_{k}=\sqrt{\frac{E_{s}}{n_{T}}} \mathbf{H}_{k} \mathbf{s}_{k}+\mathbf{n}_{k}
$$

where $\mathbf{s}_{k}=\left[s_{k, 1} s_{k, 2} \ldots s_{k, n_{T}}\right]^{T}$ is the vector of signals of transmitted over the $k^{\text {th }}$ tone, and $\mathbf{y}_{k}=\left[y_{k, 1} y_{k, 2} \ldots y_{k, n_{R}}\right]^{T}$ is the vector of samples received over the $k^{\text {th }}$ tone. $\mathbf{H}_{k}$ is the $n_{R} \times n_{T}$ channel matrix seen by the $k^{\text {th }}$ tone whose entries are the DFT of the time domain channel matrices $\mathbf{G}_{l}$ as follows

$$
\mathbf{H}_{k}=\sum_{l=0}^{L-1} \mathbf{G}_{l} e^{-j 2 \pi \frac{k l}{n_{C}}}
$$

The main advantage of OFDM is its robustness against frequency selective fading channels, which is obtained by converting the channel into several flat fading subchannels. The main disadvantage of OFDM is that the sum of sinusoidals (tones) within the symbol duration produces a large peak-to-average power ratio (PAPR). This large (10-12 dB) PAPR can not be tolerated by regular power amplifiers or other nonlinear devices such as optical fibers and laser diodes. Many techniques are used in the literature to reduce the PAPR and the research on the reduction of the PAPR ratio is very active[56][57][58][59][60][61]. 


\section{Chapter 3}

\section{Capacity of Space-Time Channels}

The capacity of a communication channel is the maximum error-free data rate that the channel can support. Claude Shannon derived the capacity equation for additive white Gaussian noise (AWGN) channels in 1948 in his famous paper "A mathematical theory of communication" [62]. The capacity of MIMO channels, where signals suffer from fading while traveling through the space domain, was developed only during the last decade. The capacity of a MIMO channel depends on many factors such as the availability of channel knowledge at the transmitter and/or the receiver, and the nature of the channel (e.g., static or time-variant, frequency flat or selective fading, Rayleigh or Ricean, etc.).

\subsection{Capacity of MIMO Channels}

The capacity of a deterministic MIMO channel with $n_{T}$ transmit antennas and $n_{R}$ receive antennas is given by [3][4][34]

$$
C=\max _{\mathbf{R}_{\mathbf{s}} \mid \operatorname{Tr}\left(\mathbf{R}_{\mathrm{ss}}\right)=n_{T}} \log _{2} \operatorname{det}\left(\mathbf{I}_{n_{R}}+\frac{\rho}{n_{T}} \mathbf{G R}_{\mathrm{ss}} \mathbf{G}^{H}\right) \mathrm{bps} / \mathrm{Hz}
$$

where $\mathbf{R}_{\mathbf{s s}}=\mathrm{E}\left[\mathbf{s s}^{H}\right]$ is the covariance matrix of the transmitted vector $\mathbf{s}, \rho$ is the average received SNR of a SISO link, $\mathbf{I}_{n_{R}}$ is an $n_{R} \times n_{R}$ identity matrix and $\mathbf{G}$ is the channel matrix. The covariance matrix of $\mathbf{s}$ must satisfy $\operatorname{Tr}\left(\mathbf{R}_{\mathbf{s}}\right)=n_{T}$ in order to constrain the total average energy transmitted over a symbol period. If the channel state information (CSI) is not known at the transmitter, so no transmit beamforming is possible, and the signals within the transmitted vector $\mathbf{s}$ are independent and have equal power, then $\mathbf{R}_{\mathrm{ss}}=\mathbf{I}_{n_{T}}$ and the capacity equation reduces to 


$$
C=\log _{2} \operatorname{det}\left(\mathbf{I}_{n_{R}}+\frac{\rho}{n_{T}} \mathbf{G G}^{H}\right) \mathrm{bps} / \mathrm{Hz}
$$

In (3.2), $\mathbf{G G}^{H}$ can be decomposed as

$$
\mathbf{G G}^{H}=\mathbf{Q} \mathbf{\Lambda} \mathbf{Q}^{H}
$$

where $\mathbf{Q}$ is an $n_{R} \times n_{R}$ Hermitian matrix satisfying $\mathbf{Q}^{H} \mathbf{Q}=\mathbf{Q} \mathbf{Q}^{H}=\mathbf{I}_{n_{\mathrm{R}}}$, and

$$
\boldsymbol{\Lambda}=\operatorname{diag}\left(\lambda_{1}, \lambda_{2}, \cdots, \lambda_{n_{R}}\right)
$$

is a diagonal matrix containing the eigenvalues of $\mathbf{G G}^{H}$ arranged in descending order, with $\lambda_{i}=0$ for $i>\operatorname{rank}(\mathbf{G})$. Equation (3.2) can be reduced to [63]

$$
C=\log _{2} \operatorname{det}\left(\mathbf{I}_{n_{R}}+\frac{\rho}{n_{T}} \boldsymbol{\Lambda}\right)
$$

where $\left[\mathbf{I}_{n_{R}}+\frac{\rho}{n_{T}} \boldsymbol{\Lambda}\right]$ is a diagonal matrix whose diagonal elements are $1+\frac{\rho}{n_{T}} \lambda_{i}$. Equation (3.5) further reduces to

$$
C=\sum_{i=1}^{n_{B}} C_{i}=\sum_{i=1}^{n_{B}} \log _{2}\left(1+\frac{\rho}{n_{T}} \lambda_{i}\right)
$$

where $n_{B}=\operatorname{rank}(\mathbf{G})$.

Equation (3.6) implies that the MIMO channel is composed of $n_{B}$ separate (orthogonal) spatial subchannels (data pipes or SISO links) each with a capacity of

$$
C_{i}=\log _{2}\left(1+\frac{\rho}{n_{T}} \lambda_{i}\right)
$$

We conclude from (3.6) that the employment of multiple antennas at both ends of the wireless link opens several subchannels that can be orthogonalized in space and hence used to transfer several symbols simultaneously on the same frequency band. 
Practically, wireless channels are randomly faded channels and this makes the capacity a random variable. The most commonly used statistical measures of this random variable is the ergodic capacity and the outage capacity [3][4][64].

The ergodic capacity is the ensemble average of the achieved data rate of a signal transmitted through an ergodic channel, so

$$
\bar{C}=\mathrm{E}\left\{\sum_{i=1}^{n_{b}} \log _{2}\left(1+\frac{\rho}{n_{T}} \lambda_{i}\right)\right\}
$$

Figure 3.1 shows how the ergodic capacity increases with increasing the number of antennas. Compared to a SISO link, only a little increase is achieved in the ergodic capacity when the number of antennas is increased at one end only, while a dramatic increase is achieved with increasing the number of antennas at both ends. For a $20 \mathrm{~dB}$ SNR at the receiver (over normalized channel), the SISO link has an ergodic capacity of $7 \mathrm{bps} / \mathrm{Hz}$, a $4 x 1 \mathrm{MISO}$ link gives $7.5 \mathrm{bps} / \mathrm{Hz}$, while we get $26 \mathrm{bps} / \mathrm{Hz}$ from a 4x4 MIMO link and $38 \mathrm{bps} / \mathrm{Hz}$ from a 6x6 MIMO link. To get this data rate from SISO link, an increase of $10^{5}$ SNR is needed. Clearly huge improvements can be obtained from MIMO systems.

The second useful measure of the capacity of a randomly faded channel is the outage capacity, which is defined as the capacity that is guaranteed with a certain reliability level (outage probability) [64][65]. For example, a $15 \%$ outage capacity of $C_{\text {out }}$ means that the instantaneous capacity will be at least as large as $C_{\text {out }}$ in $85 \%$ of channel realizations. Figure 3.2 depicts the cumulative density function (CDF) of the capacity and shows the $15 \%$ outage capacity $\left(C_{\text {out }}\right)$ as well as the ergodic capacity.

If the channel state information is unknown at the transmitter, all transmitted signals have to be of equal energy. This is a suboptimal situation because the spatial subchannels are usually of unequal power gain (unequal eigenvalues) which implies unequal power to be transmitted from each antenna to match the different sizes of the data pipes. The sum of all eigenvalues of $\mathbf{G G}^{H}$ is the total power gain of the channel matrix $\mathbf{G}$. Among all channel matrices with equal power transfer gain, the channel matrix that has equal 


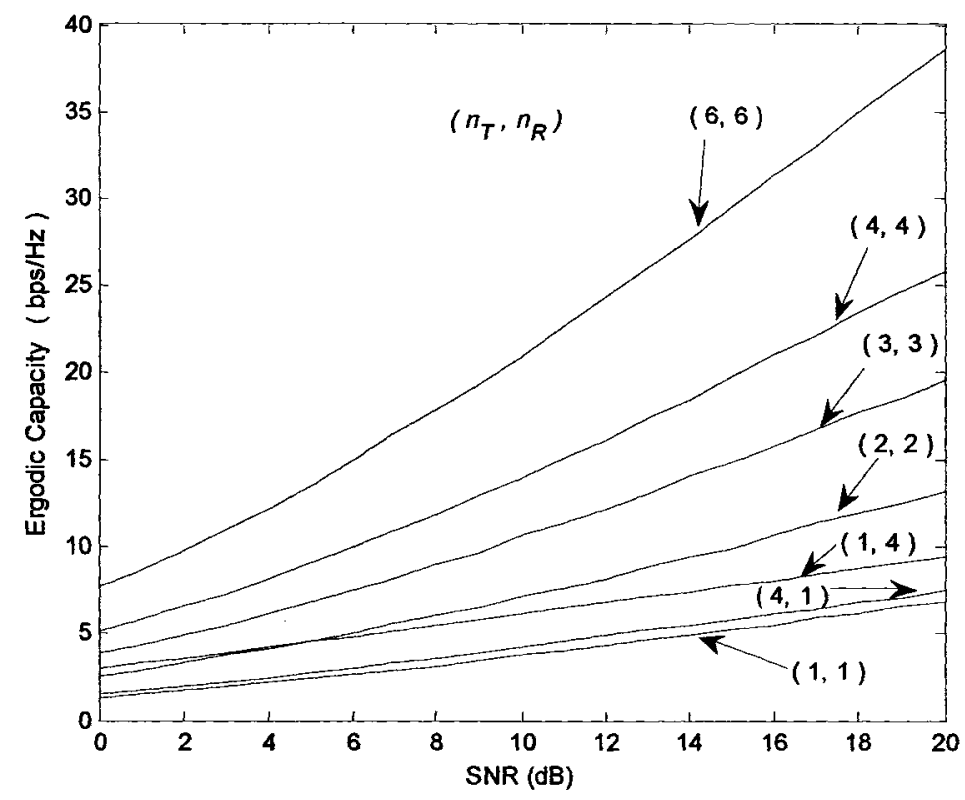

Figure 3.1. Ergodic capacity of an uncorrelated channel for different antenna configurations, with unknown CSI at the transmitter.

eigenvalues is the one that has the maximum capacity. The channel matrix $\mathbf{G}$ that has equal eigenvalues is an orthogonal matrix.

\subsubsection{Capacity with CSI Known at the Transmitter}

When the CSI is perfectly known at the transmitter, linear processing at both the transmitter and the receiver can be employed to access the spatial modes and transmit a number of symbols equal to the rank of $\mathbf{G}$ simultaneously and on the same frequency band. This can be performed by using beamforming on the channel matrix $\mathbf{G}$ of the MIMO signal model,

$$
\mathbf{y}=\mathbf{G s}+\mathbf{n} \text {. }
$$

By singular value decomposition, $\mathbf{G}$ can be decomposed as [66]

$$
\mathbf{G}=\mathbf{U} \boldsymbol{\Sigma} \mathbf{V}^{H}
$$




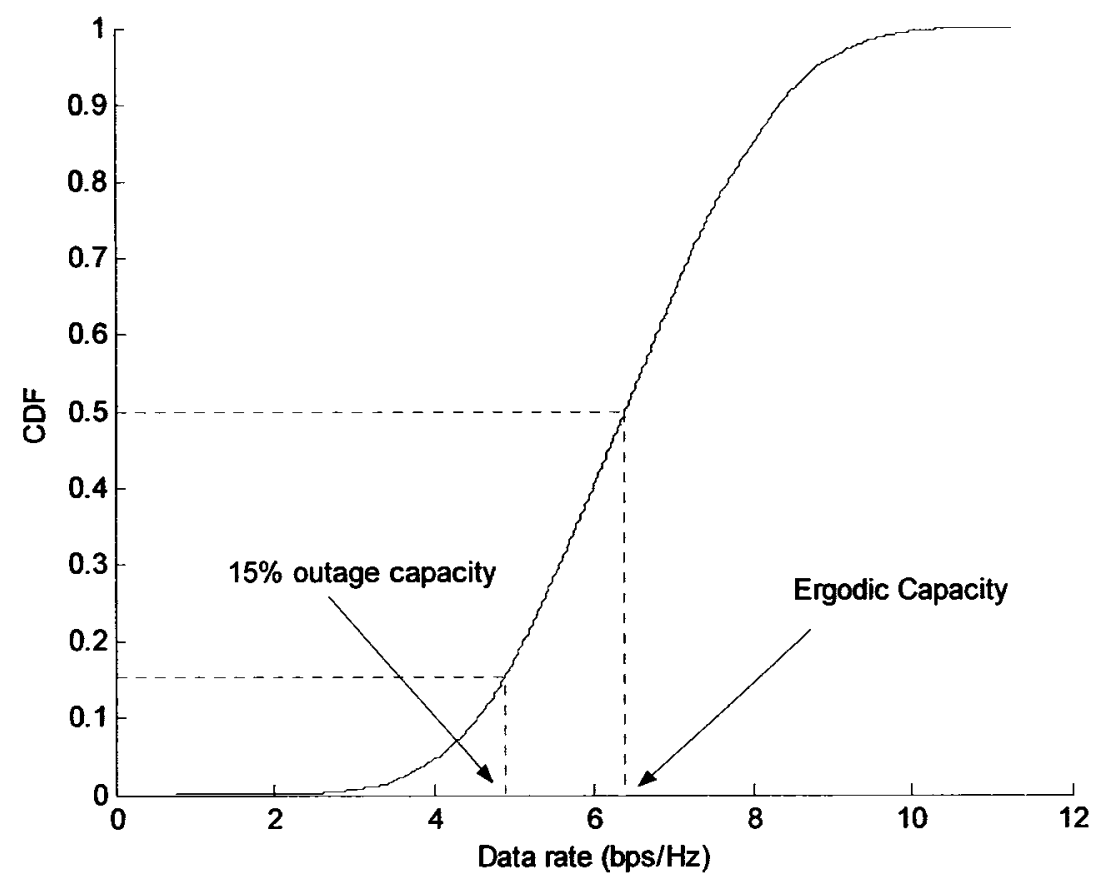

Figure 3.2. Ergodic and 15\% outage capacities of an uncorrelated channel.

where $\mathrm{U}$ and $\mathrm{V}$ are $n_{R} \times n_{R}$ and $n_{T} \times n_{T}$ unitary matrices, respectively, and

$$
\boldsymbol{\Sigma}=\operatorname{diag}\left(\sigma_{1}, \sigma_{2}, \cdots, \sigma_{n_{B}}\right)
$$

where $\sigma_{i}=\sqrt{\lambda_{i}}$ is the $i^{\text {th }}$ singular value of $\mathbf{G}$. The eigen beamforming is performed by projecting the vector of signals $\mathbf{s}$ on a new coordinate system defined by the orthonormal eigenvectors comprising the columns of $\mathbf{V}$ and this is achieved by simply multiplying the vector of signals $\mathbf{s}$ in (3.9) by $\mathbf{V}$ at the transmitter and the received vector $\mathbf{y}$ by $\mathbf{U}^{H}$ at the receiver. By this linear processing, the effective signal model for the wireless link is

$$
\widehat{\mathbf{s}}=\mathbf{\Sigma} \mathbf{s}+\mathbf{n}
$$


By this analysis the wireless channel is decomposed into $n_{B}$ orthogonal spatial channels (i.e., $n_{B}$ SISO links) as shown in Figure 3.3. The effective signal model for each transmitted symbol is therefore

$$
\hat{s}_{i}=s_{i} \sigma_{i}+n_{i}
$$

The capacity of this system is [3][4][67]

$$
C=\sum_{i=1}^{n_{B}} \log _{2}\left(1+\frac{\rho \vartheta_{i}}{n_{T}} \lambda_{i}\right) \text { bps/Hz }
$$

where $\vartheta_{i}$ is the share of energy given to each spatial mode to maximize the data rate of the system which can be found using the water pouring algorithm [67][68].

\subsection{Spatial Diversity}

A communication system that depends on the strength of a single path normally results in poor performance in fading environments, because there is a significant probability that this path will be in a deep fade. A good way to avoid this problem is to create and exploit diversity by making the transmitted symbols propagate over several different paths, so that reliable communication is possible as long as some of the paths are strong. Diversity can significantly improve the performance over fading channels. Diversity is used in wireless channels to provide multiple versions to the transmitted signal which is suffering from random fluctuations and fading [4][10]. Diversity can be obtained from time, frequency or/and space domains. Diversity over time or/and frequency domains can be achieved using interleaving and coding. Information is coded and the coded symbols are dispersed over different coherence periods in order for the codewords to experience independent fades. The simplest approach to exploit diversity is to use receive maximum ratio combining (MRC), assuming perfect CSI knowledge at the receiver.

The diversity branches might be in the space, time or a frequency domain and are assumed independent. The received signal at the $i^{\text {th }}$ diversity branch is 


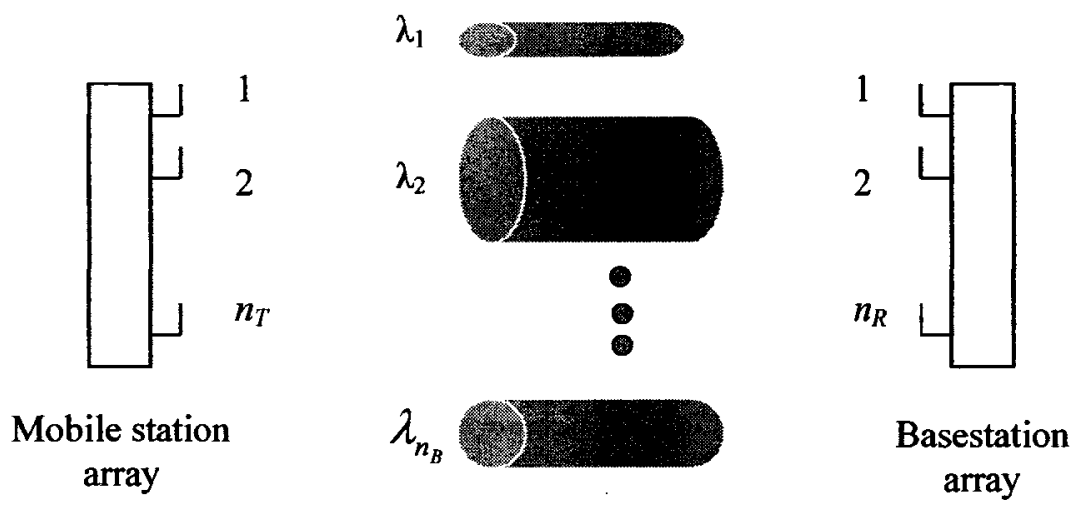

Figure 3.3. Available spatial subchannels between the transmitting and receiving arrays.

$$
y_{i}=\sqrt{\frac{E_{s}}{n_{V}}} g_{i} s+n_{i}
$$

where $s$ is the data symbol transmitted through the $n_{V}$ diversity branches, $g_{i}$ is the gain of the $i^{\text {th }}$ diversity branch, $E_{s}$ is energy of $s$ and $n_{i}$ is the noise corrupting the $i^{t h}$ branch. Using MRC, the estimate of the transmitted symbol is

$$
\hat{s}=\sum_{i=1}^{n_{v}} g_{i}^{*} y_{i}
$$

The average received SNR, over $n_{V}$ branches, is

$$
\gamma=\frac{1}{n_{V}} \sum_{i=1}^{n_{V}}\left|g_{i}\right|^{2} \rho
$$


where $\rho$ is the average received SNR of a single diversity branch.

Exploiting diversity (in time or frequency domains) is effective against fading and channel fluctuations but this benefit is not for free. To transmit a symbol through independent diversity branches, the branches have to be spaced by more than the coherence time (in time diversity) or more than the coherence frequency (in frequency diversity). The price here is a time delay or increase in frequency bandwidth. Spatial diversity, on the other hand (where symbols have to spaced by more than coherence distance) does not cost time nor frequency bandwidth plus it provides better performance (compared to time or frequency diversity) because with space diversity we get both array and diversity gains while in time or frequency techniques only diversity gain is achieved.

The deployment of multiple antennas drastically increases the channel capacity (as shown above) as they enrich the gain of wireless systems in different aspects: namely, array, diversity and spatial multiplexing gains. Diversity gain tends to reduce the fluctuations of the wireless channel due to fading, array gain results in increasing the received average SNR and the gain from spatial multiplexing is the increase in data rate, compared to a SISO channel, due to the ability of MIMO systems to multiplex several data symbols in each channel use.

\subsubsection{Receive Antenna Diversity}

To elaborate on diversity in space domain, consider a SIMO system of $n_{R}$ receive antennas as shown in Figure 3.4. Canceling unwanted co-channel interference while an array is operating in receiving mode is a very effective use of an antenna array. The process combines signals received on various antennas in such a way that the contribution due to unwanted interferences is reduced while that due to a desired signal is enhanced. The signal model for such a system is

$$
\mathbf{y}=\mathbf{g} s+\mathbf{n} .
$$

With receive MRC, the estimate of the transmitted symbol is

$$
\hat{s}=\mathbf{g}^{H} \mathbf{y}=\|\mathbf{g}\|^{2} s+\mathbf{g}^{H} \mathbf{n} .
$$




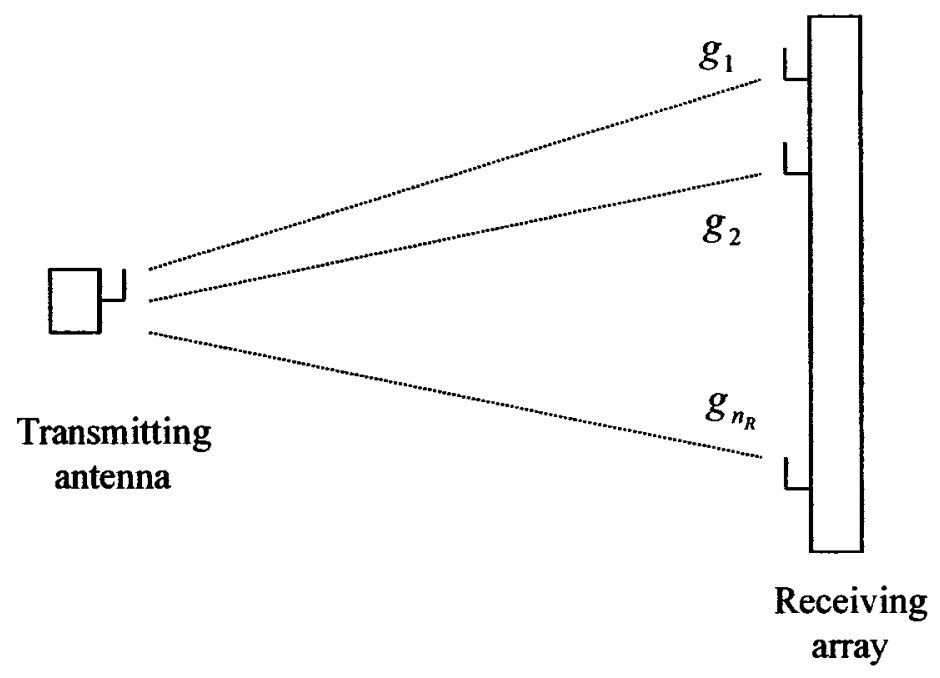

Figure 3.4. A single antenna transmitting to an array of $n_{R}$ antennas.

where $\|\mathrm{g}\|^{2}$ is the Frobenius norm of the channel vector $\mathbf{g}$. The effective SNR at the receiver, for a SISO link SNR of $\rho$, is

$$
\rho_{\text {eff }}=\|\mathbf{g}\|^{2} \rho \text {. }
$$

The average effective SNR, for a random stochastic channel $\mathbf{g}$ is

$$
\bar{\rho}_{\text {eff }}=\mathrm{E}\left\{\rho_{\text {eff }}\right\}=\mathrm{E}\left\{\|\mathrm{g}\|^{2}\right\} \rho=n_{R} \rho
$$

where we have assumed the channel to be a white channel (best case scenario), so that $\mathrm{E}\left\{\|\mathrm{g}\|^{2}\right\}=n_{R}$. We can break up the total received SNR in (3.20) into a product of two terms

$$
\rho_{\text {eff }}=\|\mathbf{g}\|^{2} \rho=n_{R} \rho \cdot \frac{1}{n_{R}}\|\mathbf{g}\|^{2}
$$


The first term, $n_{R} \rho$, corresponds to a power gain (array gain): using MRC, in an antenna array of $n_{R}$ antennas, linearly increases the power gain by $n_{R}$. The second term, $\|\mathrm{g}\|^{2} / n_{R}$, represents a diversity gain: by averaging over $n_{R}$ independent signal paths, the probability that the overall gain is small is decreased. If the channel gains are equal (or highly correlated), then we will end up having array gain only but no diversity gain. On the other hand, due to the law of large numbers, as $n_{R}$ increases the diversity gain approaches one

$$
\lim _{n_{V} \rightarrow \infty}\left\{\frac{1}{n_{V}}\|\mathbf{g}\|^{2}\right\}=1 .
$$

So the diversity gain is limited but the array gain (power gain) can be increased, theoretically, until the array picks up all the transmitted power.

\subsubsection{Transmit Antenna Diversity}

Spatial diversity can be obtained from the transmit antenna using by pre-coding the transmitted signals. Alamouti invented a simple but ingenious transmit diversity technique that has been included in the $3 \mathrm{G}$ standard [6]. In this technique, a two transmit and one receive antennas MISO system is assumed, as shown in Figure 3.5.

The symbols $s_{1}$ and $s_{2}$ are transmitted to the destination antenna over two time periods according to the code word

$$
\mathbf{S}=\left[\begin{array}{cc}
-s_{2}^{*} & s_{1} \\
s_{1}^{*} & s_{2}
\end{array}\right]
$$

which means we transmit $s_{1}$ and $s_{2}$ in the first time period and then we transmit $-s_{2}^{*}$ and $s_{1}^{*}$ in the second time period. The channel vector $\left[\begin{array}{ll}g_{1} & g_{2}\end{array}\right]^{r}$ is assumed fixed during the two time periods and known to the receiver. The received signals, $y_{1}$ and $y_{2}$, over the first and second time periods, respectively, are 


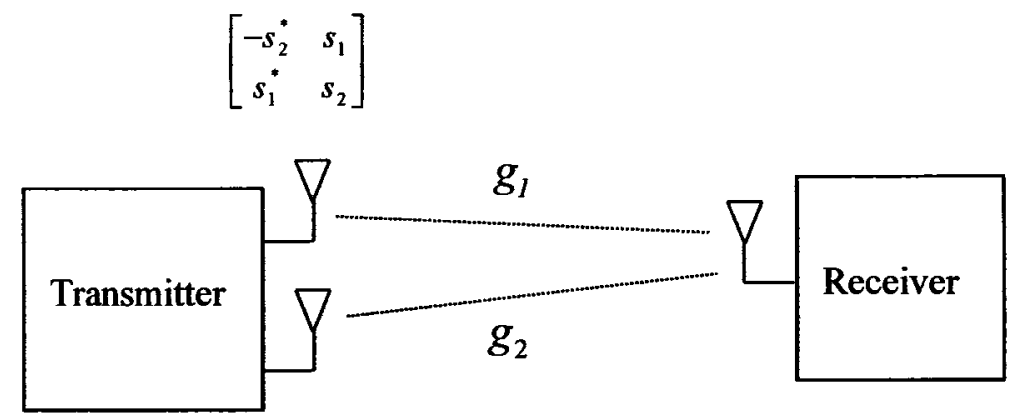

Figure 3.5. Alamouti signaling scheme.

$$
y_{1}=\sqrt{\frac{E_{s}}{2}}\left(g_{1} s_{1}+g_{2} s_{2}\right)+n_{1}
$$

and

$$
y_{2}=\sqrt{\frac{E_{s}}{2}}\left(-g_{1} s_{2}^{*}+g_{2} s_{1}^{*}\right)+n_{2},
$$

where $n_{i}$ is the noise received in the $i^{\text {th }}$ time period. The receiver decodes the transmitted symbols in two steps. It first conjugates the second received signal

$$
\left(y_{2}\right)^{*}=\sqrt{\frac{E_{s}}{2}}\left(g_{1}^{*} s_{1}-g_{2}^{*} s_{2}\right)+n_{2}^{*}
$$

This makes the effective signal model as

$$
\left[\begin{array}{l}
y_{1} \\
y_{2}^{*}
\end{array}\right]=\sqrt{\frac{E_{s}}{2}}\left[\begin{array}{cc}
g_{1} & g_{2} \\
g_{2}^{*} & -g_{1}^{*}
\end{array}\right]\left[\begin{array}{l}
s_{1} \\
s_{2}
\end{array}\right]+\left[\begin{array}{l}
n_{1} \\
n_{2}^{*}
\end{array}\right]
$$

Secondly the receiver multiplies the modified received vector with 


$$
\mathbf{G}_{e f f}=\left[\begin{array}{cc}
g_{1} & g_{2} \\
g_{2}^{*} & -g_{1}^{*}
\end{array}\right]^{H}
$$

giving

$$
\left[\begin{array}{c}
\hat{s_{1}} \\
\hat{s_{2}}
\end{array}\right]=\left[\begin{array}{cc}
g_{1} & g_{2} \\
g_{2}^{*} & -g_{1}^{*}
\end{array}\right]^{H}\left[\begin{array}{l}
y_{1} \\
y_{2}^{*}
\end{array}\right]=\left[\begin{array}{l}
\left(\left|g_{1}\right|^{2}+\left|g_{2}\right|^{2}\right) s_{1} \\
\left(\left|g_{1}\right|^{2}+\left|g_{2}\right|^{2}\right) s_{2}
\end{array}\right] .
$$

Although the channel CSI is unknown to the transmitter, full transmit diversity is extracted with this simple and smart technique.

\subsubsection{Selection Diversity}

Diversity aims at improving the quality of the wireless link. By using $n_{T}$ transmit antennas and $n_{R}$ receive antennas, a diversity order of $n_{T} \cdot n_{R}$ can be achieved [69]. Some times, complexity and cost of MIMO systems is a significant burden (due to the need of $n_{T} n_{R}$ of RF chains, including low-noise amplifiers, down converters and analog-to-digital converters). Also it might not be possible to install a large number of antennas in the mobile station handset (although it is now becoming less of a burden as digital signal processing becomes cheaper and more powerful).

Due to these reasons, a selection of a subset of antennas out of the total number of antennas, at one or both ends, is an area of ongoing research. The price paid to counteract the above mentioned problems is a reduction in system performance. But as we shall see next, this price is insignificant (compared to a full diversity system) when using proper antenna selection criteria $[26][70][71][72]$.

\subsubsection{Transmit and Receive Selection Diversity}

The selection of $L_{T}$ out of $n_{T}$ antennas at the transmitter side corresponds to selecting $L_{T}$ out of $n_{T}$ columns of the channel matrix $\mathbf{G}$, and the selection of $L_{R}$ out of $n_{R}$ antennas at the receiver side corresponds to selecting $L_{R}$ out of $n_{R}$ rows of the channel matrix $\mathbf{G}$ 


$$
\mathbf{G}=\left(\begin{array}{ccc}
g_{11} & \cdots & g_{1 n_{T}} \\
\vdots & \ddots & \vdots \\
g_{n_{R} 1} & \cdots & g_{n_{R} n_{T}}
\end{array}\right)
$$

The newly selected subchannel matrix $\tilde{\mathbf{G}}$ is of size $L_{R} \times L_{T}$. The maximum possible number of combinations of the subchannel matrices $\tilde{\mathbf{G}}$ is

$$
\left(\begin{array}{l}
n_{T} \\
L_{T}
\end{array}\right)\left(\begin{array}{l}
n_{R} \\
L_{R}
\end{array}\right)
$$

Different selection criteria are used in the literature to select the best subchannel matrix. One antenna selection criterion is to find the maximum singular value of each subchannel matrix and then select the subchannel matrix with the largest singular value [73]. That is, choose

$$
\tilde{\mathbf{G}}_{\text {selected }}=\arg \max _{\tilde{\mathbf{G}} \in \mathrm{S}(\mathbf{G})}\left\{\max _{i}\left(\tilde{\sigma}_{i}\right)\right\}
$$

where $S(\mathbf{G})$ is the set of all possible subchannel matrices and $\tilde{\sigma}_{i}$ is a singular value of the subchannel matrix $\tilde{\mathbf{G}}$.

Another selection criterion is to search for the subchannel matrix that gives the maximum capacity of the wireless link using for the targeted SNR, $\rho$ [26]. That is, choose

$$
C_{\max }=\arg \max _{\tilde{\mathbf{G}} \in \mathbf{S}(\mathbf{G})}\left\{\log _{2} \operatorname{det}\left[\mathbf{I}_{L_{R}}+\frac{\rho}{L_{T}} \tilde{\mathbf{G}} \tilde{\mathbf{G}}^{H}\right]\right\}(b p s / H z)
$$

Once the transmit and the receive antennas are selected (from the index of the rows and columns of the selected subchannel matrix), a pre-filter at the transmitter and a postfilter at the receiver are designed according to the desired transmit/receive scheme, a pure diversity system or a spatial multiplexing system. 
In a pure diversity scheme, one symbol is transmitted in each channel use. This symbol is made, by the pre-filter and the post-filter, to propagate through the designated spatial diversity branches. Because of the channel knowledge at both the transmitter and the receiver, the signal model, on the selected antennas, is

$$
\mathbf{y}=\tilde{\mathbf{G}} \mathbf{w} s+\mathbf{n}
$$

where $s$ is the $1 \times 1$ transmitted symbol, $\tilde{\mathbf{G}}$ is the $L_{R} \times L_{T}$ selected subchannel matrix, $\mathbf{y}$ is the $L_{R} \times 1$ vector of received signals, $\mathbf{n}$ is a $L_{R} \times 1$ additive white Gaussian noise vector at the receiving antennas and $w$ is a $L_{T} \times 1$ transmit pre-filter vector which can be calculated in many ways depending on the transmit scheme. The optimum (pure diversity) transmission scheme is the dominant mode transmission in which $\mathbf{w}$ is made equal to the left singular vector that corresponds to the maximum singular value of the selected subchannel matrix, $\tilde{\mathbf{G}}$ [66]. Singular value decomposition gives

$$
\tilde{\mathbf{G}}=\mathbf{U}_{\tilde{\mathbf{G}}} \Sigma_{\tilde{\mathbf{G}}} \mathbf{V}_{\tilde{\mathbf{G}}}^{\mathrm{H}}
$$

with

$$
\mathbf{V}_{\tilde{\mathbf{G}}}=\left(\begin{array}{ccc}
v_{11} & \ldots & v_{1 L_{T}} \\
\vdots & \ddots & \vdots \\
v_{L_{T} 1} & \cdots & v_{L_{T} L_{T}}
\end{array}\right)=\left[\begin{array}{lll}
\mathbf{v}_{1} & \mathbf{v}_{2} & \cdots \\
\mathbf{v}_{L_{T}}
\end{array}\right]
$$

and

$$
\mathbf{U}_{\overline{\mathbf{G}}}=\left[\mathbf{u}_{1} \mathbf{u}_{2} \cdots \mathbf{u}_{L_{R}}\right]
$$

The prefilter $\mathbf{w}$ is

$$
\mathbf{w}=\mathbf{v}_{\boldsymbol{i}}
$$

where $\mathbf{v}_{i}$ is the left singular vector that corresponds to the largest singular value. At the receiver, the estimated transmitted symbol is 


$$
\hat{s}=\mathbf{u}_{i}^{\mathbf{H}} \mathbf{y}
$$

where $\mathbf{u}_{i}$ is the right singular vector that also corresponds to the largest singular value. Thus the effective signal model is

$$
\hat{s}=\sigma_{i} s
$$

This means that the symbol is transmitted through the largest data pipe (i.e., the dominant mode).

In selection diversity with a spatial multiplexing scheme, several symbols can be transmitted simultaneously from the $L_{T}$ selected antennas (instead from all of the $n_{T}$ transmit antennas) and multiplexed in space by directing the data symbols through the available orthogonal spatial subchannels. Knowledge of the CSI at both ends of the channel enables us to use singular value decomposition for this target. The maximum number of possible multiplexed symbols is equal to the rank of the subchannel matrix $\tilde{\mathbf{G}}$, as shown in Section 3.1.1. Spatial multiplexing techniques are focused on exploiting the multipaths fading while diversity-oriented techniques try to mitigate it rather than exploit it. A trade off between multiplexing and diversity is reported in [74][29].

\subsubsection{Capacity versus the Number of Selected Antennas}

The capacity of a wireless MIMO channel increases linearly with the number of the available spatial subchannels. In other words it increases with the rank of the selected subchannel matrix, $\tilde{\mathbf{G}}$, and is given by

$$
C=\log _{2} \operatorname{det}\left[\mathbf{I}_{L_{R}}+\frac{\rho}{L_{T}} \tilde{\mathbf{G}} \tilde{\mathbf{G}}^{H}\right](\text { bps } / H z) .
$$

Figure 3.6 shows the CDF of channel capacity for the case when the receiver has $n_{R}=$ 3 antennas and the transmitter has $n_{T}=8$ antennas. As the number of selected transmit antennas increases from $L_{T}=1$ to $L_{T}=8$, the curves shift to the right, indicating improved 


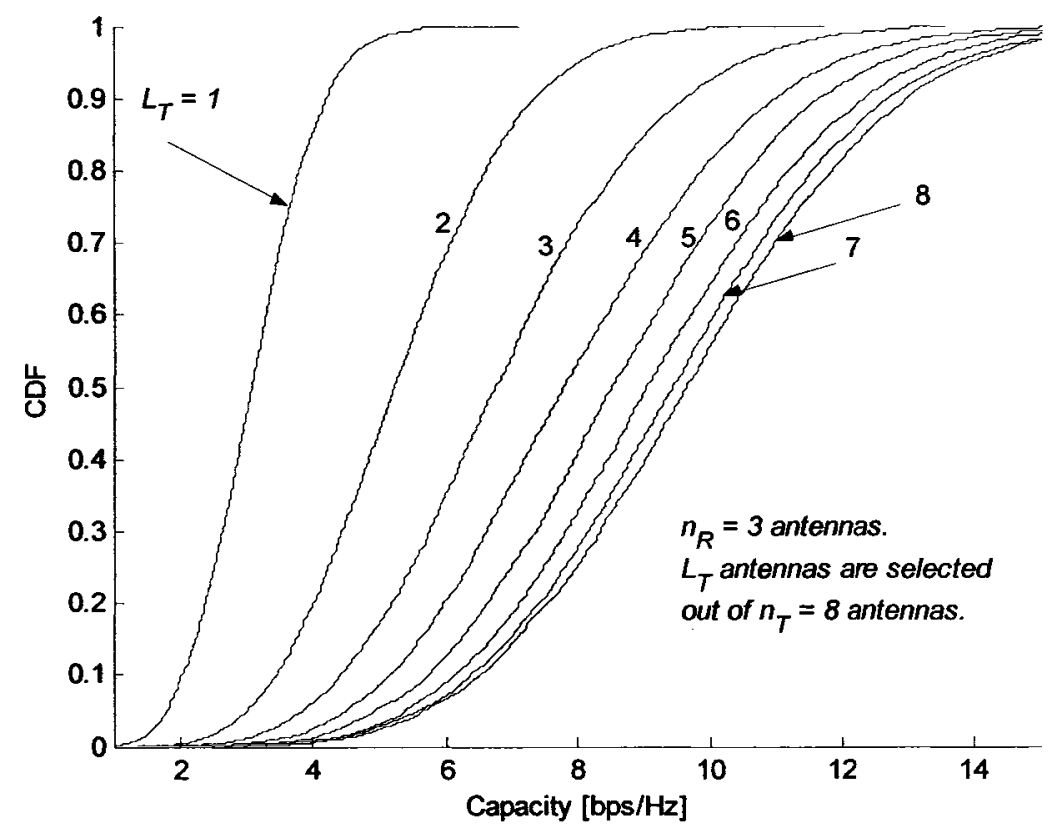

Figure 3.6. CDF of channel capacity with different number of selected basestation antennas.

system throughput. However, for each additional increase in $L_{T}$, the size of the shift diminishes. While $L_{T}$ is less than or equal to the maximum rank of $\mathbf{G}$ (i.e. $\left.L_{T} \leq \min \left(n_{R}, n_{T}\right)\right)$, the capacity increases linearly with $L_{T}$, but for larger $L_{T}$ it increases only logarithmically, since the number of spatial subchannels is no longer increasing.

Based on this observation and to reduce complexity and cost, it is advantageous to assign to each mobile user a limited number of transmit basestation antennas equal to the number of mobile user antennas. It is also advantageous, as we shall see in Chapter 4, to have the basestation antennas distributed around the mobile user. 


\subsection{Impact of Channel Parameters on the Capacity}

We consider here the effects of various channel parameters on the capacity of the system. The impact of the parameters such as channel correlations, line of site conditions, angle spread and delay spread are highlighted.

\subsubsection{Effect of Line Of Sight (LOS) on the MIMO channel capacity}

The channel with a LOS component may be modeled as [75]

$$
\mathbf{G}=\sqrt{\frac{K}{K+1}} \mathbf{G}_{\mathrm{LOS}}+\sqrt{\frac{1}{K+1}} \mathbf{G}_{\mathrm{NLOS}}
$$

where $\mathbf{G}_{\mathrm{LOS}}$ is line of sight channel which is the constant component of the channel, $\mathbf{G}_{\text {NLOs }}$ is the Rayleigh channel and $K$ is the Ricean factor which is the power ratio of the constant channel to that of the Rayleigh channel. The effect of $\mathbf{G}_{\mathrm{LOS}}$ on the ergodic capacity depends on the degree of correlations among the entries of $\mathbf{G}_{\mathrm{Los}}$. As shown in Figure 3.7, when the rank of $\mathbf{G}_{\text {Los }}$ is one, then the ergodic capacity decreases with $K$, whereas if $\mathbf{G}_{\mathrm{LOS}}$ is orthogonal then the ergodic capacity increases with the increase in $K$ (this situation occurs when the distance between the transmitting antennas is in the range of the separation distance between the transmitter and the receiver). Therefore, high- $K$ channels usually show a lower MIMO capacity. This does not mean that one would hide the antennas such that the LOS component diminishes. Low pathloss values and less fading can compensate for the loss of MIMO capacity but usually pathloss is large. High ergodic capacity is achieved with lower eigenvalues spread of the channel matrix [27][29][76].

\subsubsection{Effect of Correlation on the MIMO Channel Capacity}

The spatial fading of a MIMO channel may be correlated because of small angular spreads due to insufficient scattering and also because of smaller normalized antenna 


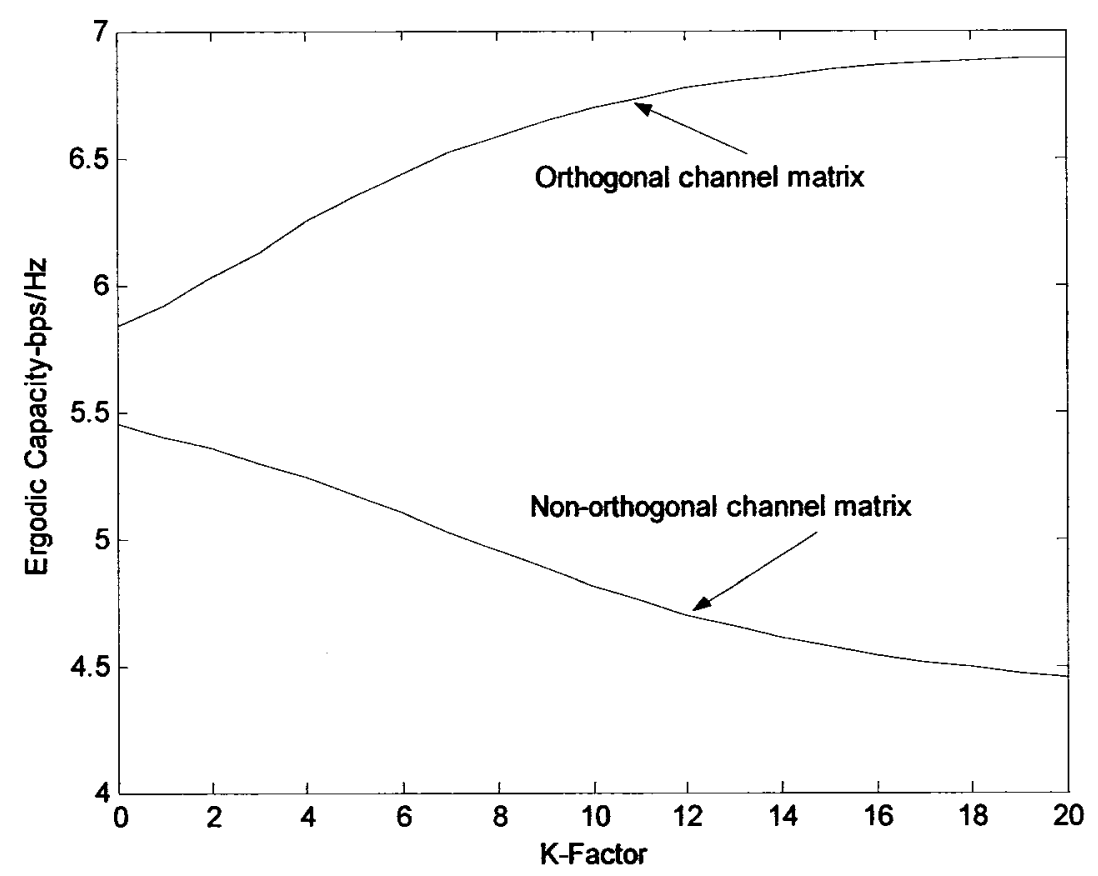

Figure 3.7. Effect of the $K$-factor on the MIMO channel capacity.

spacing. For a flat fading Rayleigh channel, the correlation in spatial fading can be modeled as [23][28][34]

$$
\mathbf{G}=\mathbf{R}_{R}^{1 / 2} \mathbf{G}_{W} \mathbf{R}_{T}^{1 / 2}
$$

The correlation matrices $\mathbf{R}_{R}$ and $\mathbf{R}_{T}$ at the transmitter and receiver, respectively are normalized such that the diagonal elements are equal to one $\left(\left[\mathbf{R}_{R}\right]_{i, i}=1,\left[\mathbf{R}_{T}\right]_{i, i}=1\right)$. Since $\mathbf{G}_{W}$ is of full rank, the rank of $\mathbf{G}$ is determined by the rank of $\mathbf{R}_{R}$ or $\mathbf{R}_{T}$.

The capacity of one correlated channel realization is [63]

$$
C=\log _{2} \operatorname{det}\left(\mathbf{I}_{n_{R}}+\frac{\rho}{n_{T}} \mathbf{G G}^{H}\right)=\log _{2} \operatorname{det}\left(\mathbf{I}_{n_{R}}+\frac{\rho}{n_{T}} \mathbf{R}_{R}^{1 / 2} \mathbf{G}_{W} \mathbf{R}_{T} \mathbf{G}_{W}^{H} \mathbf{R}_{R}^{H / 2}\right)
$$


and at high SNR, this can be approximated by

$$
C \cong \log _{2} \operatorname{det}\left(\frac{\rho}{n_{T}} \mathbf{G}_{W} \mathbf{G}_{W}^{H}\right)+\log _{2} \operatorname{det}\left(\mathbf{R}_{R}\right)+\log _{2} \operatorname{det}\left(\mathbf{R}_{T}\right)
$$

Because $\log _{2} \operatorname{det}\left(\mathbf{R}_{R}\right) \leq \mathbf{0}$ and $\log _{2} \operatorname{det}\left(\mathbf{R}_{T}\right) \leq 0$, the loss in capacity is due to the $\left[\log _{2} \operatorname{det}\left(\mathbf{R}_{R}\right)+\log _{2} \operatorname{det}\left(\mathbf{R}_{T}\right)\right]$ terms. The capacity is maximized when $\mathbf{R}_{R}=\mathbf{I}_{n_{R}}$ and $\mathbf{R}_{R}=\mathbf{I}_{n_{T}} \quad$ (i.e., when the antennas are uncorrelated), in which case $\log _{2} \operatorname{det}\left(\mathbf{R}_{R}\right)=\log _{2} \operatorname{det}\left(\mathbf{R}_{T}\right)=0$.

Figure 3.8 shows the effect of degree of correlations in $\mathbf{R}_{R}$ on the channel capacity (assuming $\mathbf{R}_{T}$ is uncorrelated). It is apparent that correlation severely decreases the ergodic capacity because it can reduce the number of spatial subchannels down to one when the rank of either $\mathbf{R}_{R}$ or $\mathbf{R}_{T}$ is one. As a result, increasing the number of antennas at both ends will lead to a capacity increase due to diversity gain only (logarithmic increase) not due to a degree of freedom gain (a linear increase is only possible with an increase of the number of spatial subchannels).

\subsubsection{Angle Spread}

The eigenvalue spread of the channel matrix is proportional to the cluster angle spread. The more clusters we have the less spread of the eigenvalues. Therefore a large total angle spread gives high ergodic capacity [29][41][45].

\subsubsection{Delay Spread}

Delay spread can offer advantages over flat-fading MIMO channels because the presence of delay spread can improve both the multiplexing gain and outage properties. This is because the delay spread increases the total angle spread [23][27][29]. It was reported by 


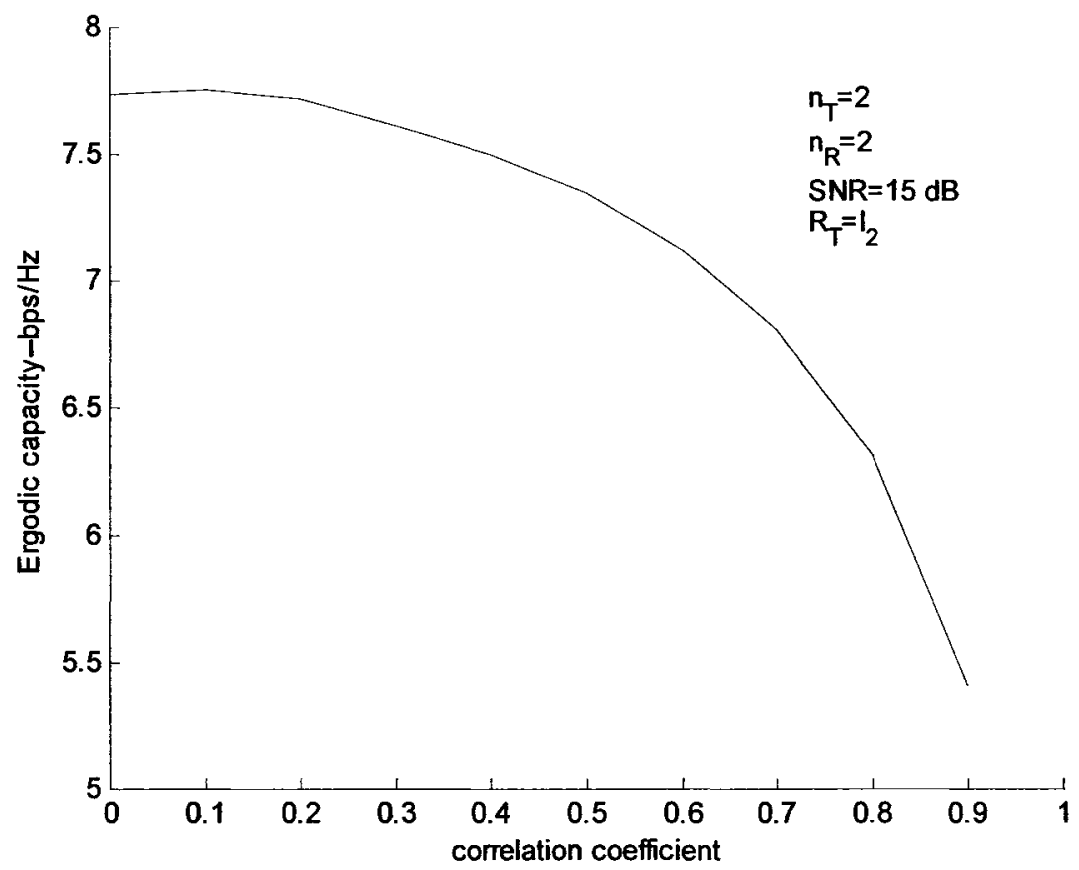

Figure 3.8. Decrease in ergodic capacity due to increase in channel correlation.

Gesbert et al. that MIMO delay spread channels may provide advantages over flat fading channels not only in outage capacity but also in ergodic capacity because it provides higher diversity gain and higher spatial multiplexing gain [27]. 


\section{Chapter 4 \\ Selection Diversity in a MIMO Channel with Distributed Antennas}

Significant enhancement in channel capacity, in a distributed arrays scenario, can be achieved by employing a selection strategy (in space and frequency domains) that aims to reduce the correlation between the signals induced at the selected antennas (i.e., by reducing the correlation between the entries of the channel matrix). In a single user propagation scenario, we propose a novel transmit antenna and OFDM tone selection algorithm that attempts to decorrelate the MIMO channel and optimize its power transfer gain. With these algorithms we are able to demonstrate the benefits of distributing the basestation antennas on the channel ergodic capacity. Improving the spatial properties of a MIMO channel by distributing antenna arrays around and closer to the user greatly enhances the system performance.

Distributing the basestation antennas enlarges the space domain and improves the spatial properties of the MIMO channel. Such a configuration may offer the mobile user a better chance of selecting, in each tone, a subchannel matrix with a better power transfer gain, decreases the channel matrix correlations, and averages the effects of shadowing. We investigate the performance of antenna and tone selection in a frequency selective fading MIMO channel with distributed base station antennas in two channel environments; an uncorrelated Rayleigh distributed MIMO channel and a correlated Ricean distributed MIMO channel.

\subsection{Channel Model}

The distributed base station antenna configuration, as shown in Figure 4.1 and previously described in Section 2.3, is considered. There are $n_{P}$ antenna arrays, with $n_{L}$ antennas each, distributed around the microcell. All of the arrays are connected via optical fiber to a central base station where all signal processing is performed. The mobile station is composed of $n_{R}$ antennas. The system seen now by the mobile user and the basestation is 


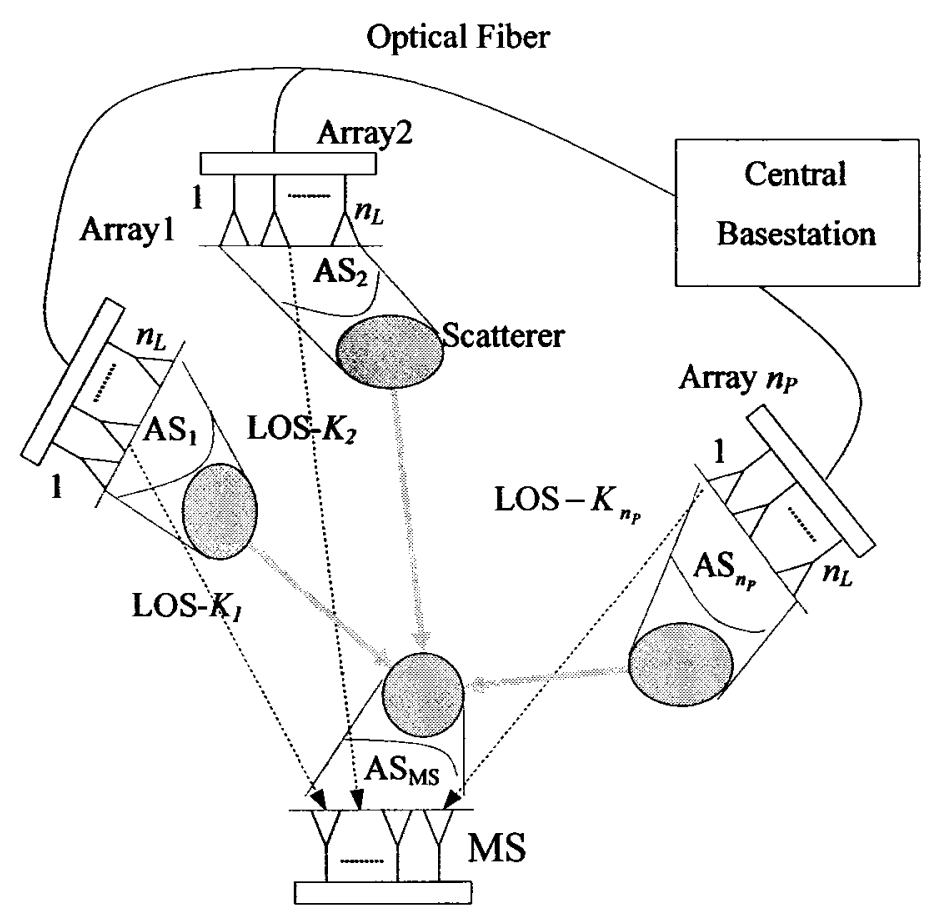

Figure 4.1. Distributed antenna arrays with a single mobile user.

a MIMO system model composed of $n_{P} n_{L}$ antennas at the base station side and $n_{R}$ antennas at the mobile user side. The user, while moving around the microcell, is surrounded by $n_{P}$ arrays. Some of the antenna arrays may have a good channel matrix while others may have a bad channel matrix.

The signal model is represented in the frequency domain as

$$
\mathbf{y}_{k}=\mathbf{H}_{k} \mathbf{x}_{k}+\mathbf{w}_{k}
$$

where $\mathbf{x}_{k}$ is the vector of symbols transmitted over the $n_{P} n_{L}$ transmit antennas on the $k^{\text {th }}$ tone, $\mathbf{y}_{k}$ is the vector of received samples from the $n_{R}$ receive antennas, and $\mathbf{w}_{k}$ is a zero mean complex Gaussian noise vector with $\mathrm{E}\left[\mathbf{w}_{k} \mathbf{w}_{k}^{H}\right]=\sigma_{\mathrm{w}}^{2} \mathbf{I}_{n_{R}} \cdot \mathbf{H}_{k}$ is the MIMO channel matrix seen by the $k^{\text {th }}$ tone and found using (2.38), 


$$
\mathbf{H}_{k}=\left[\mathbf{h}_{1}^{k} \mathbf{h}_{2}^{k} \cdots \mathbf{h}_{n_{\mathbf{p}} n_{\mathbf{L}}}^{k}\right]=\left(\begin{array}{ccc}
h_{1,1} & \cdots & h_{1, n_{\mathrm{P}} n_{L}} \\
\vdots & \ddots & \vdots \\
h_{n_{R}, 1} & \cdots & h_{n_{R}, n_{p} n_{L}}
\end{array}\right)
$$

The vector $\mathbf{h}_{i}^{k}$ in the downlink transmission represents the channel gains (for tone $k$ ) between a transmitting antenna $i$ and all receiving antennas of the user.

\subsection{Antenna Selection}

Averaging of shadowing is achieved using the selection among the space dimension (different antennas) and the frequency dimension (different tones) so the user is assigned the best available channel. Depending on channel conditions, the user may get an adaptive data rate according to the needed quality of service (QoS), for example, by adaptive tone assignment and adaptive signaling schemes (like space-time coding or spatial multiplexing).

The main factor controlling the capacity of a MIMO system is the rank of the channel matrix. The rank is actually the number of virtual uncoupled data pipes (spatial subchannels) between the transmitter and the receiver. The size of each pipe corresponds to a certain eigenvalue (power gain) of the matrix, so the number of non-zero eigenvalues (or non-zero singular values) is the rank, and the sum of all the eigenvalues is the total channel power transfer gain.

In our propagation scenario each tone sees a channel composed of $n_{P} n_{L}$ antennas at the base station side and $n_{R}$ antennas at the user side. Only a few antennas can be implemented at the mobile station side (due to limited space), while many antennas can be used at the basestation side. Since the maximum rank is $\min \left(n_{R}, n_{P} n_{L}\right)$, the maximum portion of the capacity is due to a sub-channel matrix of size $n_{R} \times n_{R}$ while the rest of the capacity is due to the array and diversity gain. The capacity increases linearly with an increase in the number of data pipes (rank) between the base station and the mobile station and it increases logarithmically with the number of antennas (array gain) (see 
Section 3.2.3.2). We conclude from this discussion that it is advantageous to select at least $n_{T}=n_{R}$ antennas out of $n_{P} n_{L}$ antennas at the base station side.

Although the rank is the main factor controlling the capacity of a MIMO system, it is a crude measure of this capacity. A more refined measure is the eigenvalues spread, which can be characterized by the condition number. Among the channels with the same total power gain, the one which has the highest capacity is the one with all eigenvalues being almost equal. More generally, the less spread out the eigenvalues are the larger the capacity [23]. The ergodic capacity of a MIMO link is, generally, given by [34]

$$
C=\mathrm{E}\left\{\log _{2} \operatorname{det}\left[\mathbf{I}_{n_{\mathbf{R}}}+\frac{\rho}{n_{T}} \mathbf{H H}^{H}\right]\right\}
$$

where $\rho$ is the average SNR at each antenna, and $n_{T}$ and $n_{R}$ is the number of transmit and receive antennas, respectively. It is assumed here that the power is divided equally between the uncorrelated transmitted signals. At low SNR the above relation can be approximated by [34]

$$
C=\mathrm{E}\left\{\log _{2} \operatorname{det}\left[1+\frac{\rho}{n_{T}}\|\mathrm{H}\|^{2}\right]\right\}
$$

while at high SNR [34]

$$
C=\operatorname{rank}(\mathbf{H}) \log _{2}\left(\frac{\rho}{n_{T}}\right)+\mathrm{E}\left\{\sum_{i=1}^{\operatorname{rank}(\mathbf{H})} \log _{2}\left(\lambda_{i}\right)\right\},
$$

where $\lambda_{i}$ are the eigenvalues of $\mathbf{H H}^{H}$. We see from the above relations that the capacity of the low SNR case is determined mainly by the power gain of the channel, while in the high SNR case it is determined by the rank and condition number of $\mathbf{H}$ (the eigenvalue distribution). The condition number, for a two geographically separated antennas transmitting to an array of antennas, is [41]

$$
\frac{\lambda_{\max }}{\lambda_{\min }}=\sqrt{\frac{1+\beta}{1-\beta}}
$$


where $\lambda_{\max }$ and $\lambda_{\min }$ are the maximum and minimum eigenvalues, respectively, of the channel matrix, $\beta=\left|\mathbf{e}_{R}\left(\theta_{1}\right)^{H} \mathbf{e}_{R}\left(\theta_{2}\right)\right|$, and $\theta_{1}$ and $\theta_{2}$ are the angles of arrival of the signals from transmit antenna 1 and 2 , respectively. When $\beta \cong 1, \mathbf{G}$ is ill-conditioned, otherwise it's well-conditioned. In other words, the less aligned the spatial signatures of the transmitting antennas, the better conditioned the channel matrix. According to the above physical model, it is possible to get a well-conditioned subchannel matrix when the user is assigned (by a selection algorithm) antennas from geographically separated basestation arrays. This will ensure less correlation between the transmit antennas and will reduce the condition number of the subchannel matrix (i.e. it will become well-conditioned) which will result in enhancement of channel capacity.

As mentioned in Section 3.3.1, the presence of a LOS component may reduce the ergodic capacity of the MIMO channel, but due to the distribution of arrays around the user, it is possible for the user to be assigned antennas in the space and frequency domains that maximize the channel capacity (lower effective $K$-factor and lower correlation and condition number).

It is advantageous to select at least $n_{T}=n_{R}$ antennas at the base station side. The selected antennas don't have to be located in the same array. By this selection scenario the user will be assigned the best conditioned sub-channel matrix in terms of correlation, condition number, $K$-factor and power transfer gain.

The user might also be assigned $n_{K}$ out of $n_{C}$ tones of the OFDM symbol. An antenna assigned to the user doesn't have to transmit on all the $n_{K}$ tones, meaning that each tone, of the $n_{K}$ tones, might have its own well-conditioned channel matrix. The tones assigned to the user need not be transmitted from the same set of antennas, as shown in Figure 4.2, for example. Those tones that are transmitted from different antennas will be combined correctly at the receiving side if the difference between their time of arrival is less than the guard time interval of the OFDM symbol. As illustrated in Figure 4.3, when the delay difference $\left|d_{k}-d_{l}\right| / c$ is less than OFDM guard time, no ISI or ICI will occur [22]. The addition of the time shifted signals creates a diversity advantage for the user. To the user, 


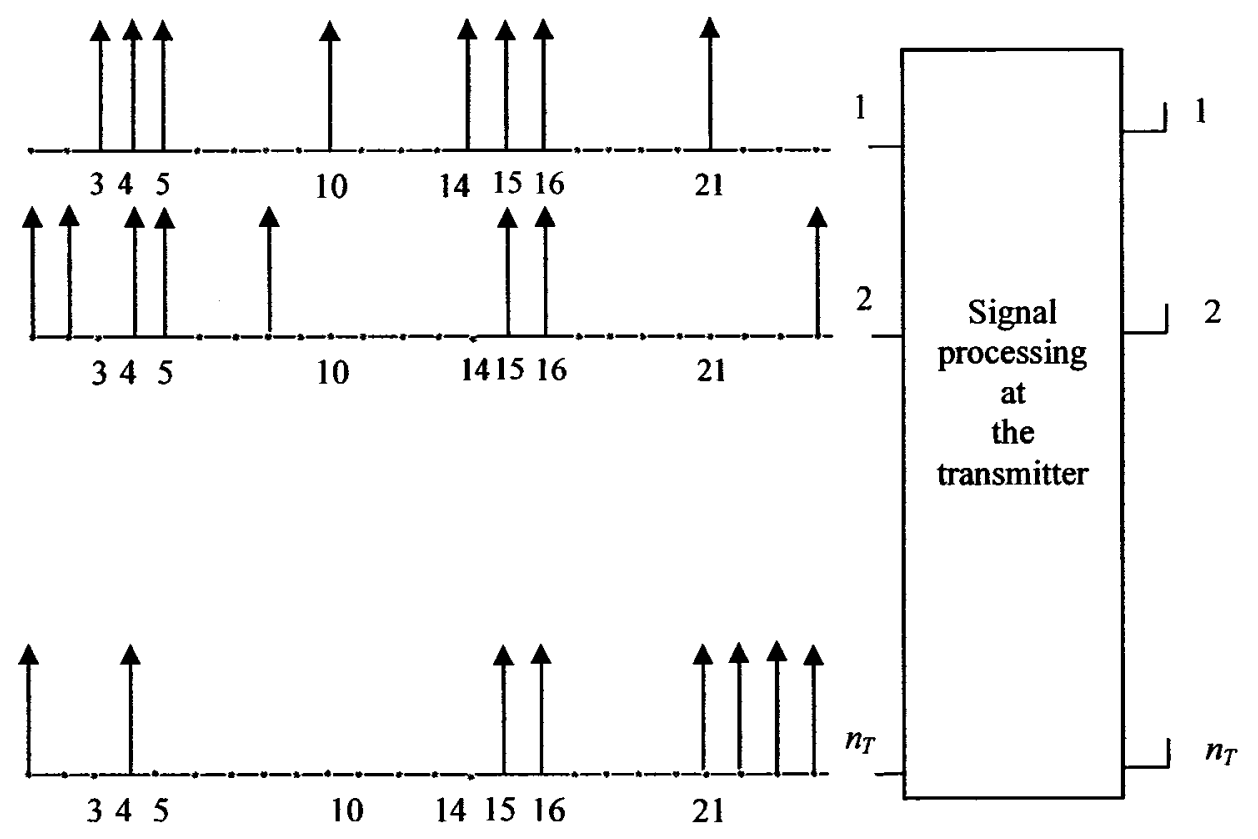

Figure 4.2. OFDM tones transmitted from different antennas.

this situation is equivalent to a two-ray multipath channel. The probability that the sum of both signals has an unacceptable low power because of shadowing and flat fading is much lower than the probability that one of the individual signals is too weak.

\subsection{Antenna and Tone Selection Algorithms}

The optimum selection method of antennas and tones is the one based on the capacity criterion, because the relationship between phase values of the channel matrix elements are important decisive factors for channel capacity[25][26][77]. SNR based Power transfer gain is a suboptimal selection criterion, but much less complex.

\subsubsection{Capacity Based Selection Algorithm}

To select the antennas and tones according to the capacity-based selection criterion, the following process is used. For each tone, $k$, select the set of $n_{T}$ transmit antennas out of $n_{P} n_{L}$ that gives the maximum capacity according to 


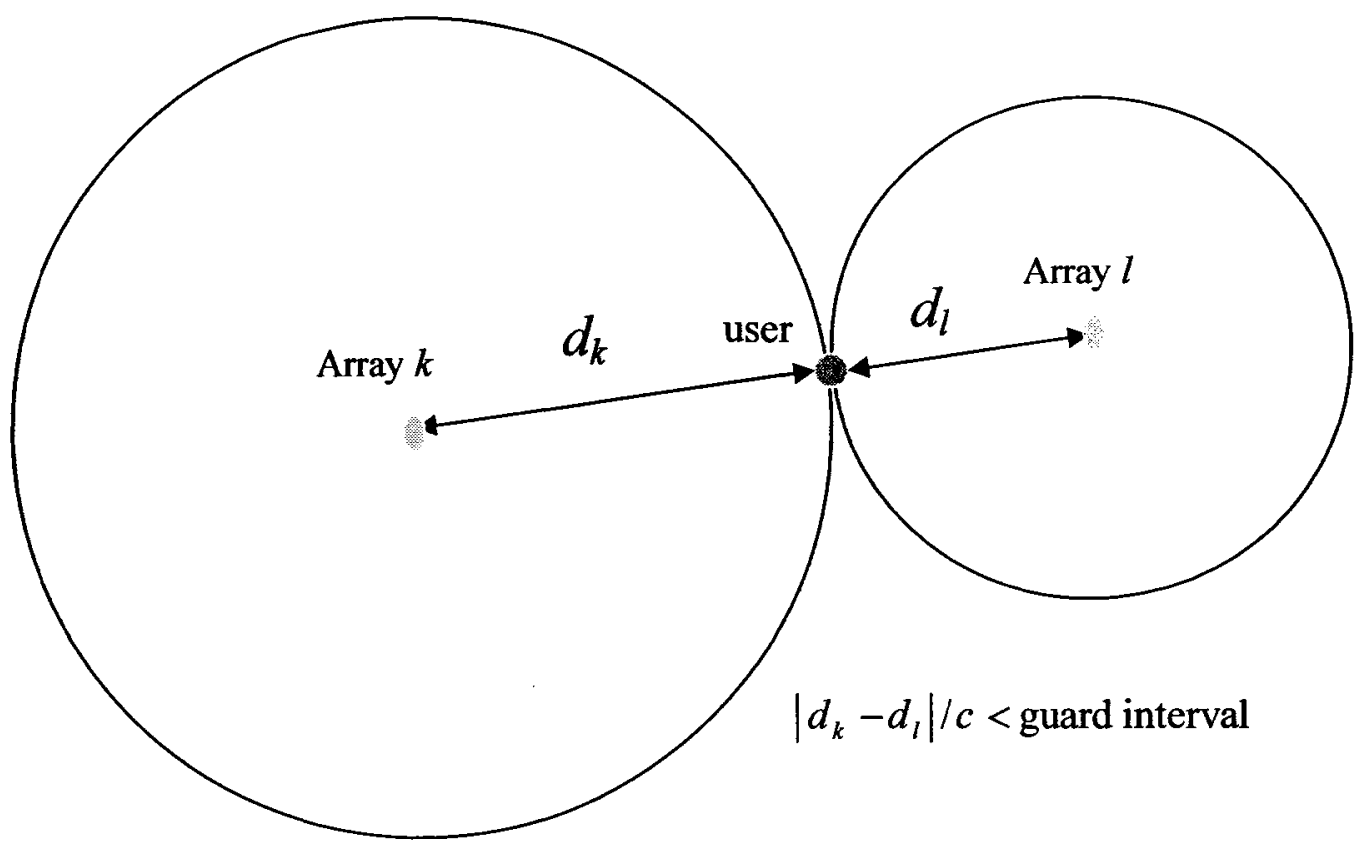

Figure 4.3. Two antennas transmitting the same OFDM tone to a mobile

$$
\mathrm{C}_{k, \max }=\max _{\tilde{\mathbf{H}}_{k} \in \mathbf{S}\left(\mathbf{H}_{k}\right)} \log _{2} \operatorname{det}\left[\mathbf{I}_{n_{R}}+\frac{\rho}{n_{T}} \tilde{\mathbf{H}}_{k} \tilde{\mathbf{H}}_{k}^{H}\right]
$$

where $\rho$ is average SNR and $\mathrm{S}\left(\mathbf{H}_{k}\right)$ is the set of all subchannel matrices consisting of $n_{T}$ columns of $\mathbf{H}_{k}$. Then, select the $n_{K}$ tones that give the highest capacity.

This approach is an optimal selection algorithm but it is based on a long complicated search that requires the evaluation of $\left(\begin{array}{c}n_{P} n_{L} \\ n_{T}\end{array}\right)$ sets of capacity calculations. This can be computationally prohibitive, especially with a large number of basestation antennas. 


\subsubsection{SNR Based Selection Algorithm}

A much faster, but suboptimal, approach is to make the selection based on the channel power transfer gain seen by the antennas (i.e., the SNR based selection criterion). The power transfer gain from the $m^{\text {th }}$ antenna, for tone $k$, is

$$
\mathrm{p}_{k, m}=\sum_{n=1}^{n_{R}}\left|h_{n m}^{k}\right|^{2}
$$

For each tone, the selection algorithm selects the $n_{T}$ antennas that have the highest power transfer gains. Then, the $n_{K}$ tones that give the highest combined transfer gain,

$$
\mathrm{P}_{k}=\sum_{m=1}^{n_{T}} \mathrm{P}_{k, m}
$$

are selected, where it is assumed that $\mathrm{P}_{k, m}$ is sorted in descending order.

Although this algorithm is very simple, it is not very effective when the signals are highly correlated, because subchannel matrices with poor condition numbers tend to be selected. To address this shortcoming, we propose the following modification to the algorithm. Instead of strictly picking the $n_{T}$ antennas with the largest power transfer gains, we try to pick antennas from different arrays that have large transfer gains. If the number of required antennas for transmission $\left(n_{T}\right)$ is less than the number of arrays (i.e., $n_{T} \leq n_{P}$ ) then select the $n_{T}$ antennas from different arrays and which have maximum power transfer gain. If the number of required antennas for transmission is greater than the number of arrays (i.e., $n_{T}>n_{p}$ ) then select the first $n_{P}$ antennas from different arrays and that have maximum power transfer gain and then select the remaining $n_{T}-n_{P}$ antennas that are far apart from the previously selected antennas and that have maximum power transfer gain.

An example of assigning four tones and three base station antennas to a mobile user is shown in Table 4.1. The other unused tones might be assigned to other users (in a multiuser environment), or left unused in a single user propagation scenario and the saved transmit power reallocated to the selected tones. 


\begin{tabular}{|c|c|c|c|}
\hline $\begin{array}{c}\text { Index of tone } \\
\text { assigned to the } \\
\text { user }\end{array}$ & \multicolumn{3}{|c|}{ Antennas selected for each tone for the user } \\
\hline 15 & 1 & 3 & 6 \\
\hline 19 & 1 & 6 & 8 \\
\hline 20 & 1 & 6 & 8 \\
\hline 61 & 2 & 5 & 6 \\
\hline
\end{tabular}

Table 4.1. An example of the antennas assigned to a user in four assigned OFDM tones.

The capacity-based selection criterion gives better results than the suboptimal SNR based selection criterion because the phases of the channel matrix elements play an important role in determining the channel capacity. But the advantage of the suboptimal method is that the determination of the best antennas is much simpler (for very small loss in performance) than if we have to make a full search among all possible antenna combinations [25].

\subsection{Receiver Implementation}

Recovery of the spatially multiplexed symbols on each tone is performed according to either the zero-forcing (ZF) criterion or the minimum mean square error (MMSE) criterion. Estimates of the transmitted symbols are given by

$$
\hat{\mathbf{x}}_{k}=\mathbf{M}_{k} \mathbf{y}_{k}
$$

where $\mathbf{y}_{k}$ is the vector of received samples at tone $k$, and $\mathbf{M}_{k}$ is either [78]

$$
\mathbf{M}_{Z F, k}=\left(\mathbf{H}_{k}^{H} \mathbf{H}_{k}\right)^{-1} \mathbf{H}_{k}^{H}
$$

if zero-forcing is used, or 


$$
\mathbf{M}_{\mathbf{M M S E}, k}=\left(\mathbf{H}_{k}^{H} \mathbf{H}_{k}+\sigma_{\mathbf{W}}^{2} \mathbf{I}\right)^{-1} \mathbf{H}_{k}^{H}
$$

for MMSE.

\subsection{Simulation Results}

The main objective of the simulations is to compare antenna and tone selections in a point to multi-point MIMO channel (distributed antenna arrays), with the selection in a point-to-point MIMO channel (traditional MIMO). We consider the power transfer gain of the channel matrix as a selection criterion (SNR-based selection algorithm). In case of correlated channels we employ the modification to the algorithm, mentioned in Section 3.3.2, that aims at reducing the eigenvalue spread and the condition number of the channel matrix resulting in better channel capacity. We model the multipath fading as a tapped-delay line with 3 taps, as $\left(\begin{array}{lll}0 & -5 & -10\end{array}\right) \mathrm{dB}$, according to Stanford University interim model (SUI 3).

\subsubsection{Uncorrelated Rayleigh Fading}

We assume that the channel is frequency selective fading and it contains a rich scattering environment and that the antenna configurations (at the arrays and user sides) are in such a way that makes the signals induced on the antennas on both sides of the wireless link to be uncorrelated (as a result of uncorrelated fading). We also assume that no specular or LOS components exist in the wireless link so we are left with a pure flat fading uncorrelated channel seen by each tone and is perfectly known to both the transmitter and receiver sides. We use an OFDM symbol with $n_{C}=64$ tones and we select $n_{K}=32$ tones for each user. The tones are loaded by QPSK symbols drawn from a zero mean unit variance constellation.

Figure 4.4 shows the CDF of channel capacity for different antenna selection configurations, where $\mathrm{SNR}=10 \mathrm{~dB}$. The curve labeled 3 shows that selecting $n_{T}=3$ transmitting antennas, out of 14 , from $n_{P}=7$ distributed arrays of $n_{L}=2$ antennas each, gives significant performance improvement over the case of selecting the same number of antennas and tones but from two arrays of 7 antennas each or one array of 14 antennas 
(curves labeled 2 and 1, respectively). Increasing the number of receive antennas to 4 (and correspondingly increasing the number of transmit antennas selected), yields an additional substantial increase (curve 4).

Figure 4.5 compares the average BER versus SNR of a spatial multiplexing signaling scheme with ZF or MMSE receivers. Here, the user has 5 antennas while 3 antennas are selected from the basestation out of 14 antennas. It demonstrates that distributing more arrays around the user offers better performance. For the MMSE receiver, the curve labeled 2, in which the basestation has $n_{P}=7$ distributed arrays of $n_{L}=2$ antennas each, outperform the case, of curves labeled 1 , when the basestation has only $n_{P}=2$ arrays of $n_{L}=7$ antennas each. Same performance comparison applies to the $\mathrm{ZF}$ receiver (curve labeled 4 with curve labeled 3). In addition, MMSE provides a slight performance advantage over ZF at low SNR values. 


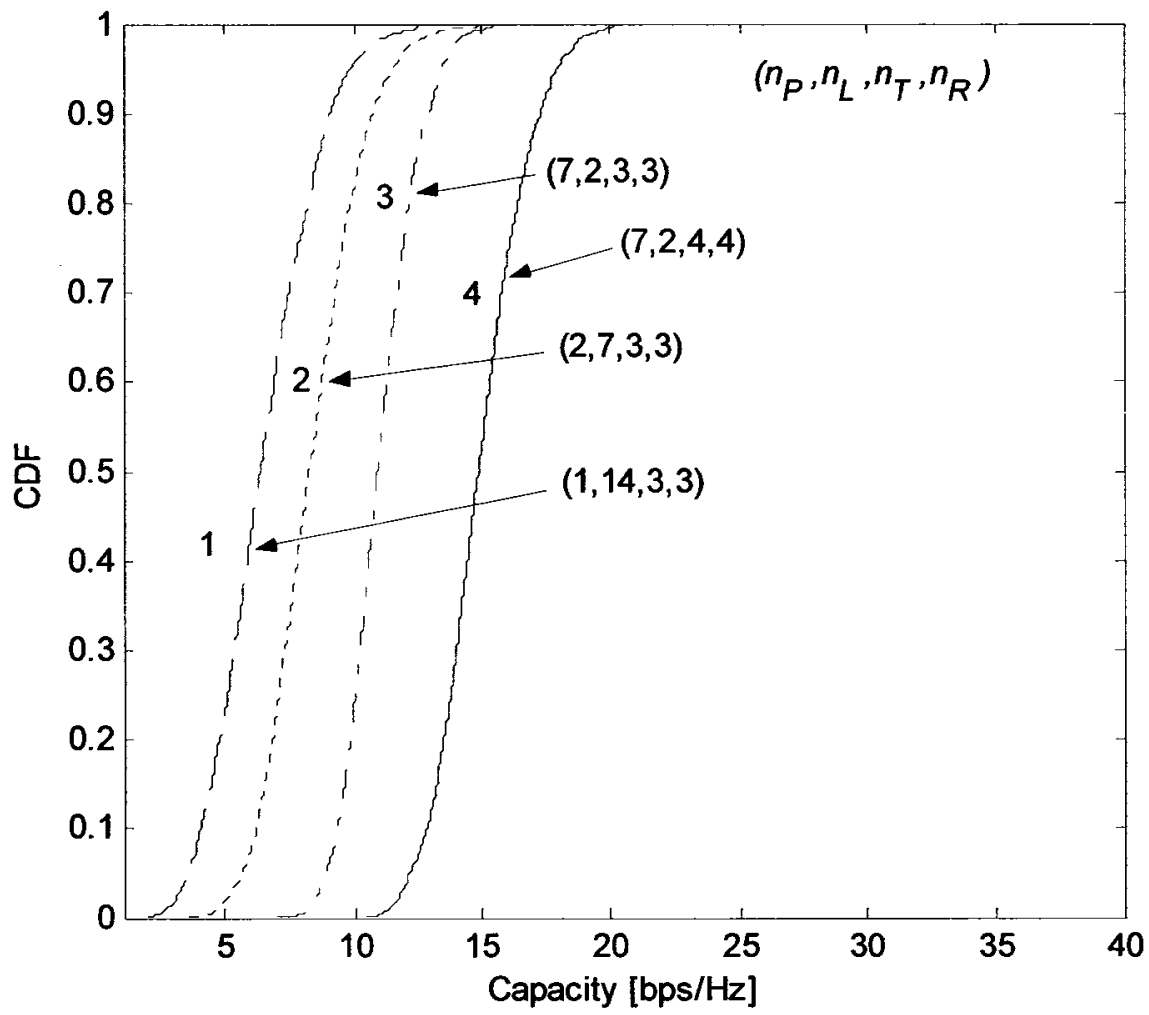

Figure 4.4. $\mathrm{CDF}$ of channel capacity for different antenna configurations $(\mathrm{SNR}=10 \mathrm{~dB})$. 


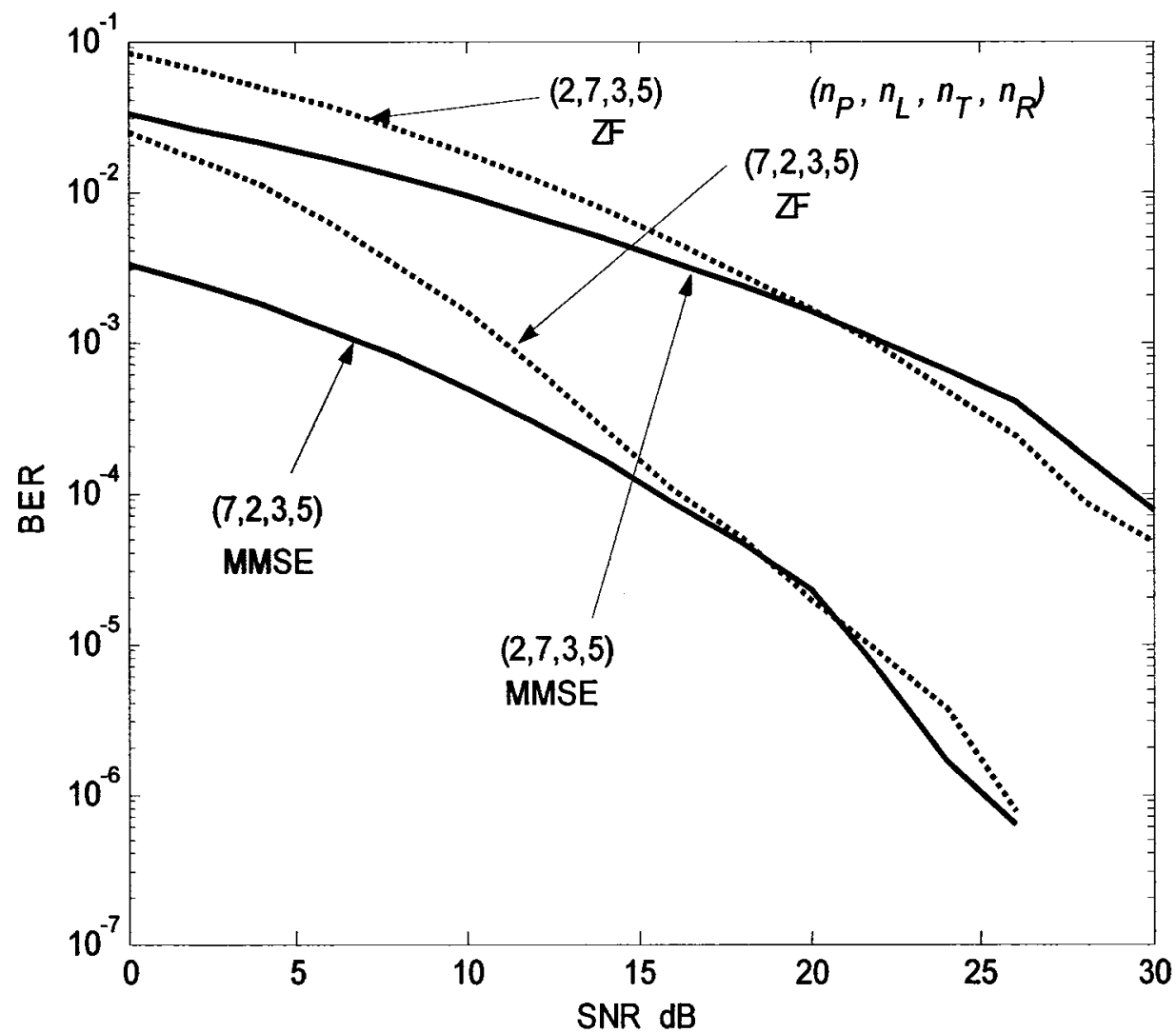

Figure 4.5. BER versus SNR of different antenna configurations using zeroforcing and MMSE receivers.

\subsubsection{Correlated Ricean Fading}

Using the SNR selection criterion, an $L$-tap correlated-Ricean channel is generated for each array (each with its angular spread, angle of departure, angular spectrum and $K$ factor), then we select for each tone the best $n_{T}$ antennas out of all basestation antennas, $n_{P} n_{L}$, according to the modified algorithm, proposed for Ricean-correlated channels, in Section 4.3.2. Figure 4.6 shows the CDF of channel capacity, Figure 4.7 shows the CDF 
of channel matrix eigenvalues, Figure 4.8 shows the CDF of channel matrix condition number, and Figure 4.9 shows the ergodic capacity versus SNR.

Curves labeled 1 to 4 in each of the figures, Figure 4.6 to Figure 4.9 , demonstrate four different antenna configurations, with fixed correlation properties and the $K$-factor for all arrays, as follows; $K$-factor $=10 \mathrm{~dB}$, Gaussian angular spectrum at the BS arrays with angular spread of 10 degrees, uniform angular spectrum at the MS with angular spread of 30 degrees, antenna spacing of $0.5 \lambda_{c}$ and the power delay profile (PDF) $=\left[\begin{array}{ll}0-5 & -10\end{array}\right] \mathrm{dB}$.

The number of selected OFDM tones is 32 out of 64 tones, the number of antennas at the mobile user is 3 , while 3 out of 24 antennas are selected at the basestation side (the total number of BS antennas is fixed at 24 , while the number of arrays is varied and the number of antennas within each array is also varied accordingly)

Curves labeled 1 shows the case of selecting 3 basestation antennas out of $n_{P}=2$ arrays with $n_{L}=12$ antennas each. For this configuration, a low values of channel capacity is shown in Figure 4.6, a large eigenvalue spread is shown by the CDF of eigenvalues in Figure 4.7, a large condition number is shown in Figure 4.8 and a relatively bad ergodic capacity, versus SNR, is shown in Figure 4.9. Curves labeled 2 show the case of selecting 3 basestation antennas out of $n_{P}=4$ arrays with $n_{L}=6$ antennas each. This configuration shows some improvement in channel capacity, as shown in Figure 4.6, less eigenvalue spread, as shown in Figure 4.7, less condition number, as shown in Figure 4.8 and better ergodic capacity, vs SNR, as shown in Figure 4.9. Curves labeled 3 shows the case of selecting 3 antennas out of $n_{P}=6$ arrays with $n_{L}$ $=4$ antennas each. More improvement is shown here. 


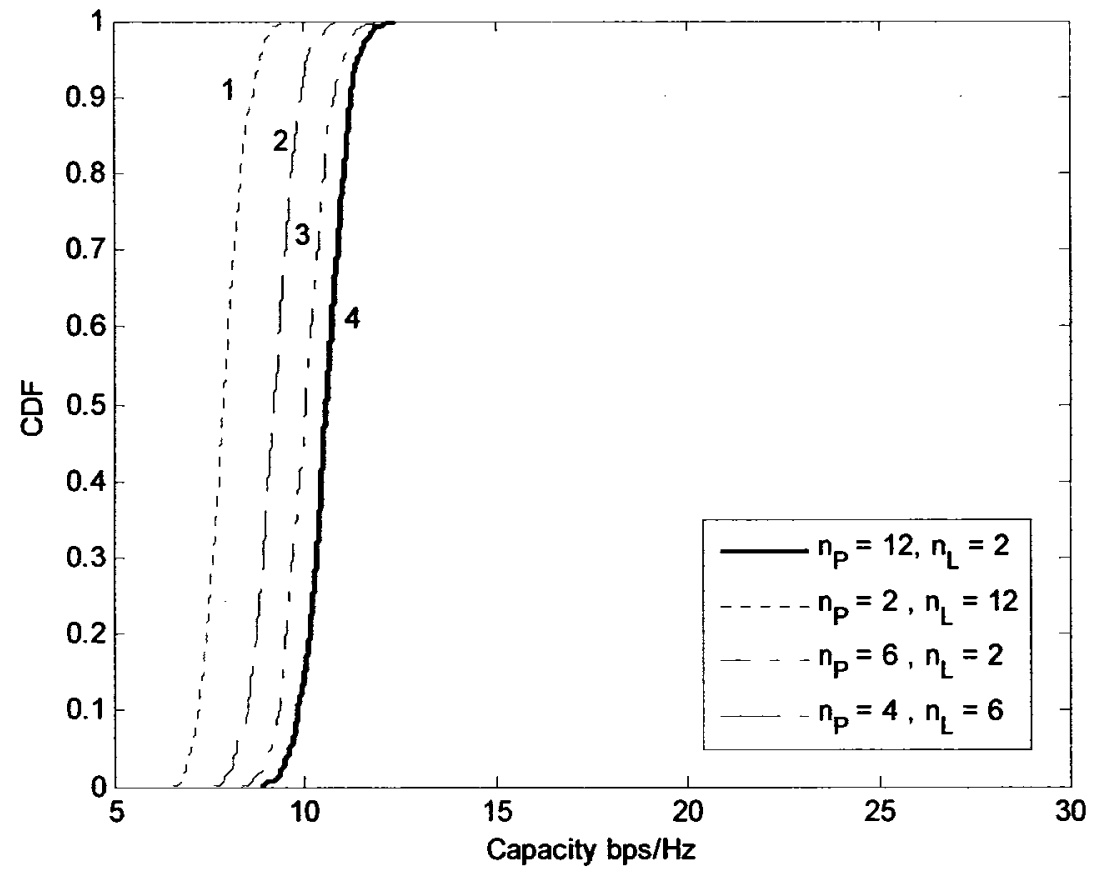

Figure 4.6. CDF of channel capacity for different antenna configurations, $\mathrm{SNR}=10 \mathrm{~dB}, K_{P}=10$.

The best performance is shown in curves labeled 4 which reflects a basestation of $n_{P}$ $=12$ arrays with $n_{L}=2$ antennas each. This configuration depicts an ergodic capacity of $11.5 \mathrm{bps} / \mathrm{Hz}$ compared to $8 \mathrm{bps} / \mathrm{Hz}$ of configuration 1 with much less eigenvalue spread and the lowest left shift of the CDF of the condition number curve. 


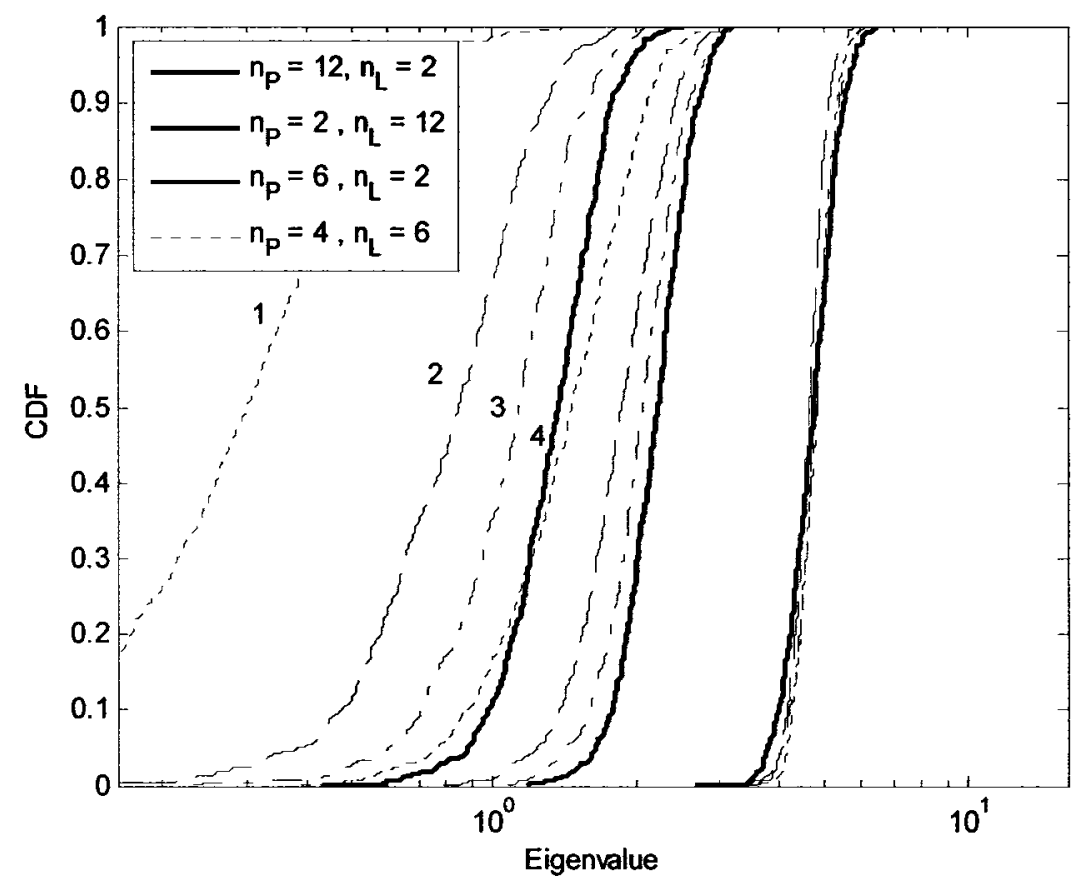

Figure 4.7. CDF of channel eigenvalues for different antenna configurations. 


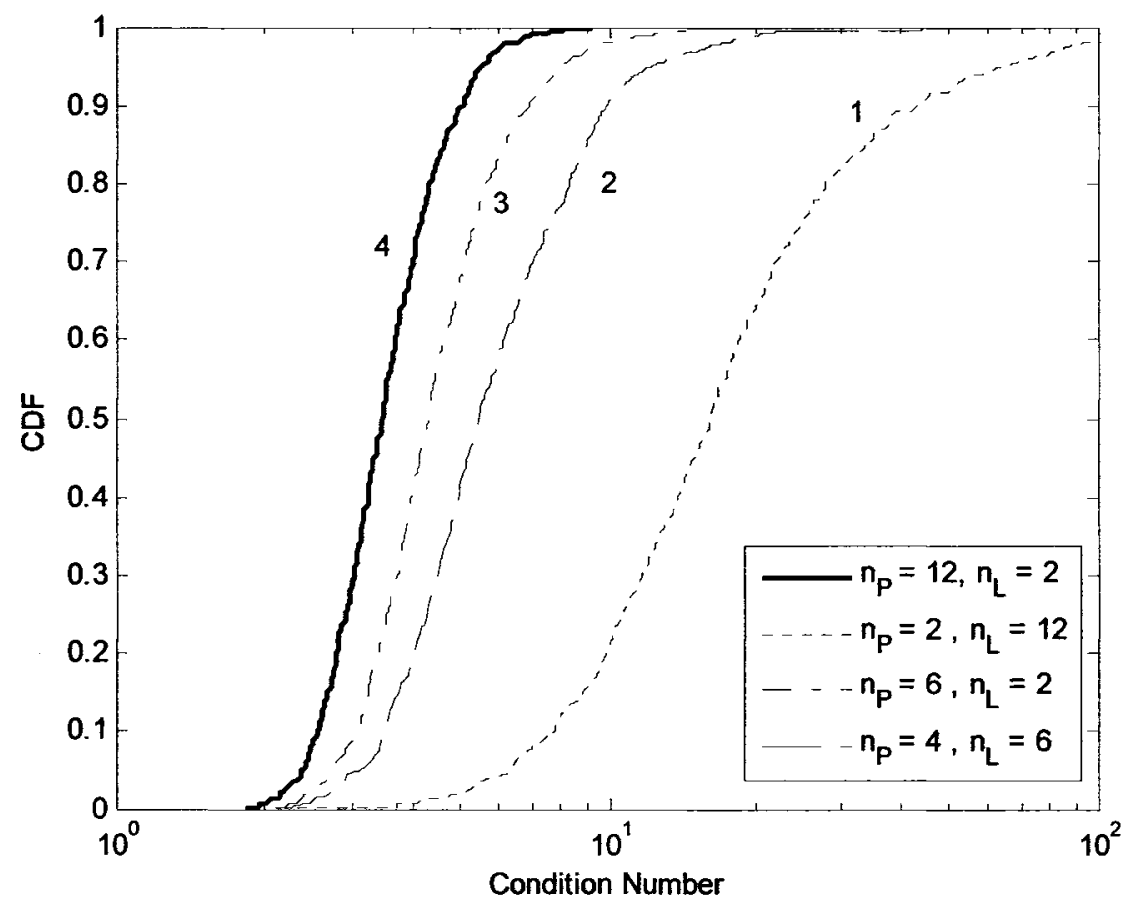

Figure 4.8. CDF of channel condition number for different antenna configurations, $\mathrm{SNR}=10 \mathrm{~dB} . \mathrm{K}_{\mathrm{P}}=10$, fixed correlation. 


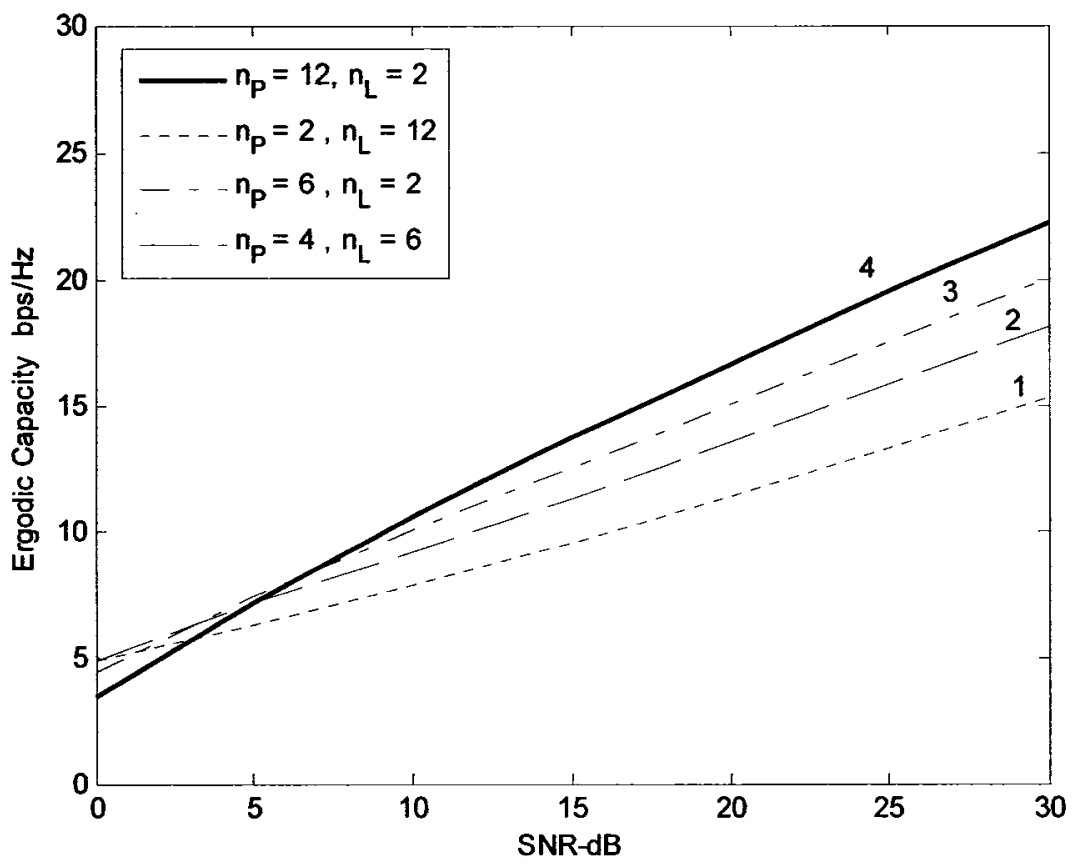

Figure 4.9. Ergodic capacity versus SNR for different antenna configurations. 
Curves labeled 1 to 5 in each of the figures, Figure 4.10 to Figure 4.12, show the effect of correlation, $K$-factor, configuration of antennas and the number of taps of the power delay profile on the channel capacity and demonstrate five different MIMO channel environments. In all cases we select for the user, with $n_{R}=3$ antennas, 3 antennas out of the 12 basestation antennas. Curves labeled 1 demonstrate a MIMO channel with a poor scattering environment represented by placing one array $\left(n_{P}=1\right)$ on the basestation tower with $n_{L}=12$ co-located antennas and with an angular spread of 2 degrees and a Laplacian angular spectrum while the $K$-factor $=10 \mathrm{~dB}$ and a 3 tap power delay profile $(\mathrm{PDF})=[0-5-10] \mathrm{dB}$. In all other cases, we distribute the basestation antennas as $n_{P}=6$ arrays with $n_{L}=2$ antennas each. In Curves labeled 2 we assume a case of rich scattering environment represented by angular spread of 30 degrees with Uniform angular spectrum at each array while the $K$-factor $=10 \mathrm{~dB}$ and a 3 tap PDF. Curves labeled 3 represent a MIMO channel similar to that in case 2 except of decreased $K$-factor to $1 \mathrm{~dB}$. Curves labeled 4 also represent a MIMO channel similar to that in case 2 except of assuming the power delay profile to have 6 taps of $[0-2-4 \cdots-10] \mathrm{dB}$. Curves labeled 5 represent a case of randomly varying the angular spread in each array between 5 to 30 degrees with uniform angular spectrum, and also randomly varying the $K$-factor of each array in the range 1 to $10 \mathrm{~dB}$ while keeping the power delay profile fixed to 3 taps for all arrays.

Comparing Curves (1) \& (2) in the three figures. We note that case 2 outperforms case 1 as shown in the improvement in channel capacity with the right shift of CDF curve in Figure 4.10 and a decrease in the condition number in Figure 4.11 and the increase of ergodic capacity, vs SNR, in Figure 4.12. Curves labeled 3 show that the reduction of the $K$ factor improves the channel capacity and reduces the channel matrix condition number. Curves labeled 4 show that a channel with larger delay spread improves channel capacity and reduces condition number. Curves labeled 5 reflects a realistic MIMO channel environment as it assumes a different scattering environment around the arrays, represented by different angular spread and different $K$-factor. A significant improvement in performance is evident as the it seems that the decrease of the angular spread in some of the arrays can be compensated by the decrease in the $K$-factor in other arrays. 
In general, the distribution of arrays enriches the scattering environment which reduces the eigenvalue spread and the opens more degrees of freedom in space for the selection algorithm which lead to clear improvement in the ergodic capacity and decreasing the ergodic condition number of the channel matrix to values close to one. 


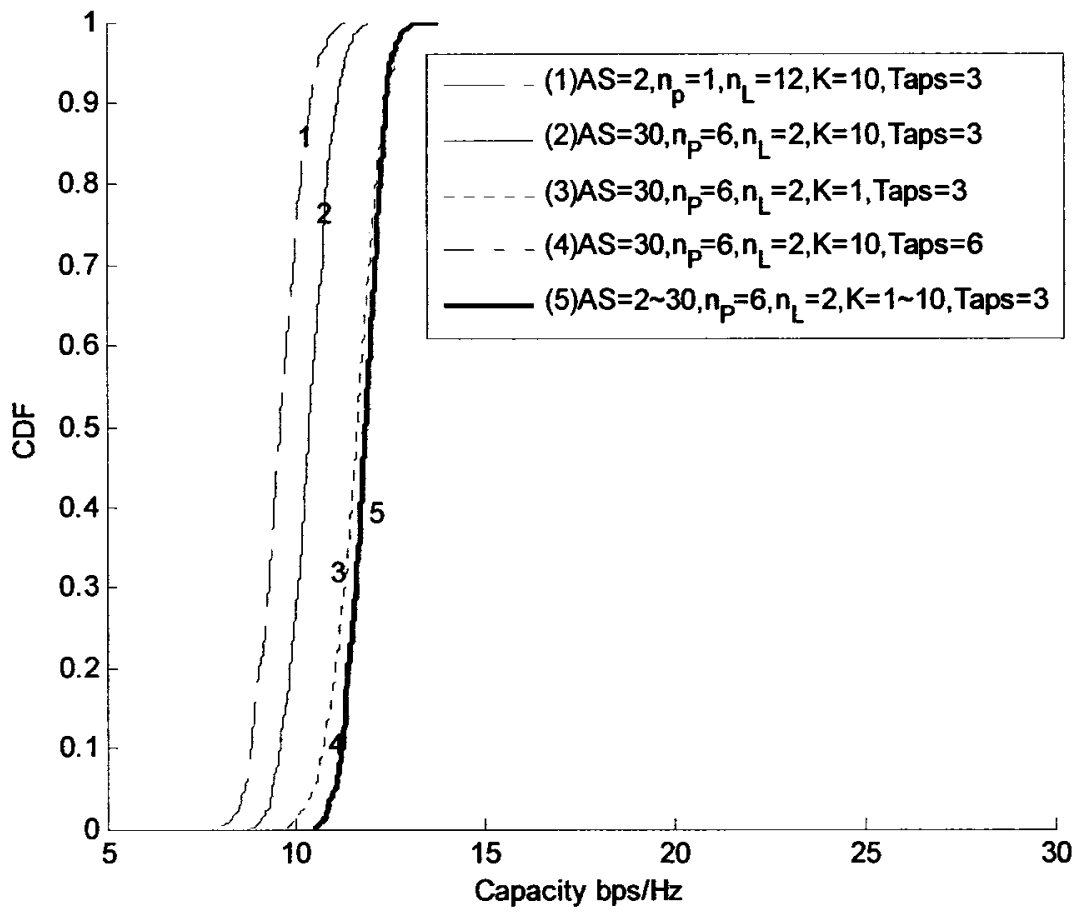

Figure 4.10. CDF of channel capacity under different antenna configurations, and different correlation and Ricean conditions. 


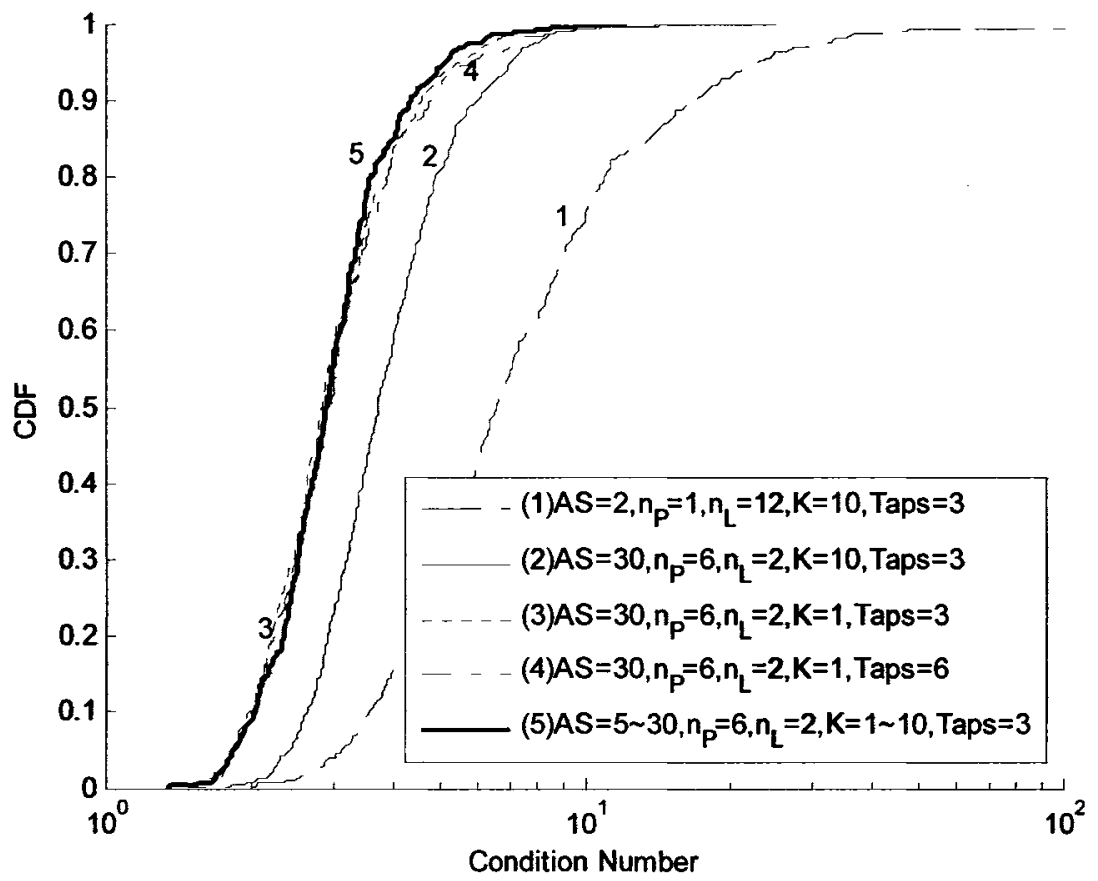

Figure 4.11. CDF of condition number under different antenna configurations, and different correlation and Ricean conditions. 


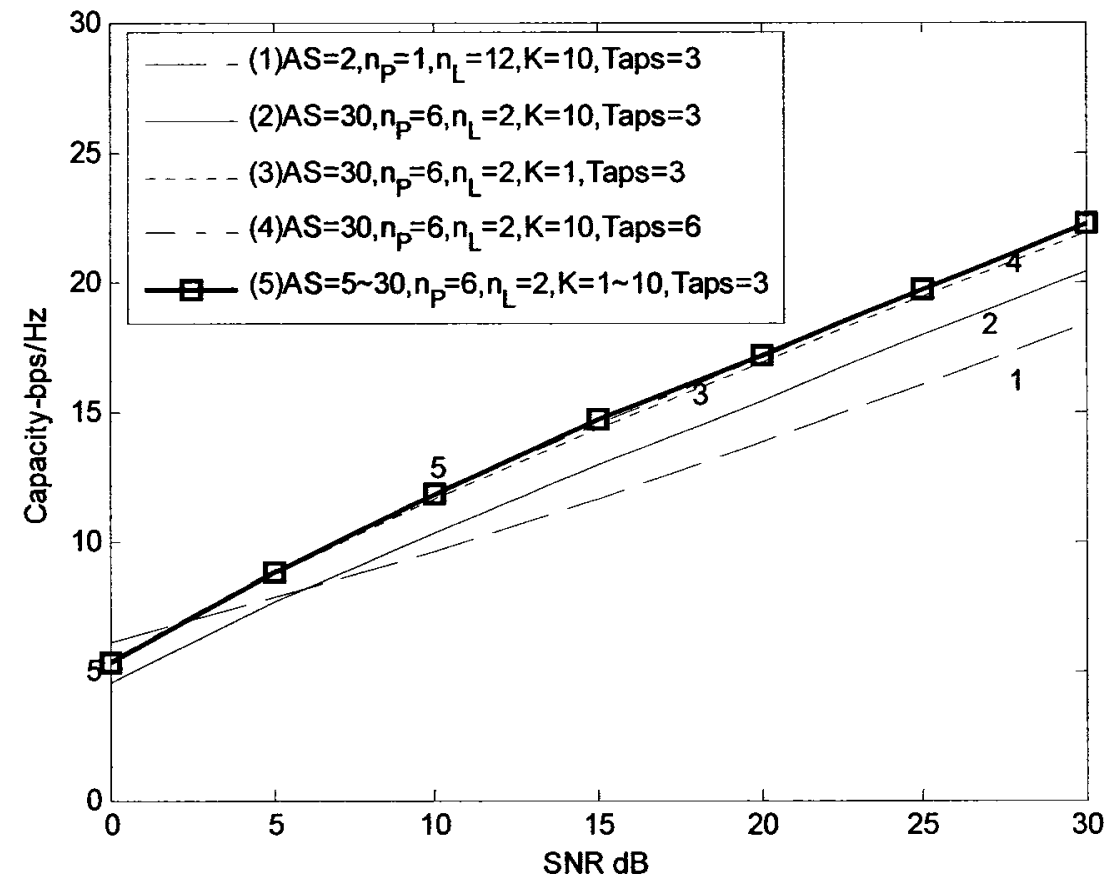

Figure 4.12. Ergodic capacity versus SNR under different channel Environments. 


\section{Chapter 5 \\ Decorrelation of Multi-user Downlink MIMO Channels}

In this chapter we shift our focus to multiuser downlink MIMO channels. We develop a decorrelation algorithm that enhances the spatial separability of the mobile users and study the role of distributing the antennas of the basestation, by using multiple arrays, on such channels. In addition to allowing spatial multiplexing and providing spatial diversity to each user, multiple antennas allow the base station to simultaneously transmit or receive data to or from multiple users at high data rates [17]. This is a consequence of the increase in degrees of freedom from having multiple antennas (see Appendix A). Several mobile users with different spatial signatures can communicate simultaneously with the basestation on the same frequency band. This allows frequency band reuse within the cell resulting in increasing spectral efficiency. Practically, the location of the users and the scattering may cause the spatial signatures of the mobile users to be almost identical. In this situation it is difficult to separate the users based on spatial properties. Because mobile users are located at different distances to the basestation, they will have different pathloss with the basestation, so the near far problem makes it even more difficult for the basestation to distinguish them. Knowledge of the channel is essential in SDMA signaling schemes otherwise the penalty is very significant in system performance.

The ability of the basestation to spatially separate several users depends on the pair wise cross-correlations between the channel matrices of the users (the inter-user correlation). In this chapter we propose an improved null steering downlink MIMOOFDM system that reduces both the inter-user correlation and the near-far problem resulting in a significant enhancement in system performance. We use the distributed antenna system, presented in Section 2.3, where several basestation multi-antenna arrays are distributed in a given area. Each array communicates with the basestation via optical fiber links, and all transmitter signal processing is performed at the basestation. Multiantenna users are spatially separated such that only a subset of the users is served by each 
tone of the OFDM symbol. The served users are selected based on an algorithm that reduces the inter-user correlations.

Distributing the arrays around the users also balances the channel matrix leading to significant reduction in the effect of the near-far problem. The channel matrix of each user is assumed correlated and Ricean distributed. Several data symbols can be spatially multiplexed to each user over each OFDM tone with high reliability and with good total system capacity. Multiple input multiple output (MIMO) systems, orthogonal frequency division multiplexing (OFDM), radio-over-fiber (RoF), and space division multiple access (SDMA) are four techniques that, when combined together, can provide very spectrally efficient data transmission and thereby meet the high speed requirements of future generations of wireless systems. Combining OFDM with MIMO provides exceptional system performance by exploiting diversity in both the frequency and space domains. SDMA is a communication technique that enables several multi-antenna mobile users to communicate with the same basestation simultaneously in the same frequency band with very little interference on each other. Additional details on SDMA and beamforming can be found in Appendix A.

\subsection{Channel Model}

We consider the channel model previously described in Section 2.3 . There are $n_{P}$ antenna arrays distributed around a microcell, each with $n_{L}$ antennas elements and each mobile user has $n_{R}$ antennas. The fading of the channel between the antennas of any of the arrays and the mobile station antennas is assumed to be frequency selective and quasistatic. The channel state information (CSI) is assumed known at the basestation (for example, by using an uplink to return channel measurements collected during the transmission of downlink training sequences). The fading statistics of any given tone within the OFDM signal is assumed to have a flat fading Ricean distribution. From site to site, the average power, the Ricean $K$-factor and the correlation (between antenna elements) changes due to different path losses, shadowing and scattering environments around the antennas. The shadowing component is assumed to have a lognormal 
distribution. The correlation properties between the antennas within each array depend on the angular spread, angular spectrum and direction of arrival or departure of the impinging wave and on the arrangement of the antennas and the spacing between them.

The angular spectrum is assumed to be Gaussian [49] or Laplacian [50] at the distributed antenna arrays of the basestation. We assume a rich scattering environment, at the user side, so the angular spectrum is assumed uniformly distributed with a wide angular spread [48]. The direction of arrival (DoA), direction of departure (DoD) and the angular spread (AS) are in general time-variant due to the movement of the mobile station and the changing of scatterers around the antennas, however they are assumed to remain fixed for the duration of the transmission of a block of data.

The $L$ channel taps defines the time-domain MIMO channel model. The $n_{C}$ point discrete Fourier transform (DFT) of these taps (for every transmit/receive antenna pair) gives the channel gains seen by each tone of the OFDM symbol (for that antenna pair). Each tone of each user will see the $n_{R} \times n_{P} n_{L}$ channel matrix

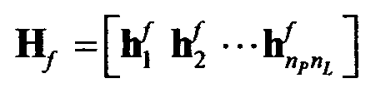

which is the frequency domain channel matrix for tone $f$. The vector $\mathbf{h}_{i}^{f}$ represents the channel gains (for tone $f$ ) between transmitting antenna $i$ and all the receiving antennas of the user.

For a multi-user propagation scenario two types of correlations have to be taken into account: the correlation between the entries of the channel matrix of each user, and the correlation between the channel matrix of a user to that of other users. To be able to separate users spatially (using SDMA techniques), its preferred that their channel matrices be less correlated. If it happens that two or more users come close to each other and the antennas of the basestation are co-located, its highly likely that their channel matrices will be highly correlated, but if the antennas of the basestation are distributed as arrays and only users with low pair-wise cross-correlations are selected in each tone, then the system performance will be significantly enhanced. 


\subsection{Downlink Null Steering Signaling Models}

We consider two downlink nullsteering signal models, a downlink nullsteering MISO signal model where each user has a single antenna, and a downlink nullsteering MIMO signal model where each user has several antennas.

\subsubsection{Signal Model of a Single Antenna Mobile Users (downlink MISO)}

We assume here that each user has just a single antenna. The frequency domain representation of the time-domain MIMO channel model $\mathbf{G}_{l}$ can be found using equation (2.38) in Section 2.4, so the $n_{P} \cdot n_{L}$ channel vector for one tone seen by the $k^{\text {th }}$ mobile user is

$$
\mathbf{h}_{k}=\left[h_{1}^{k} h_{2}^{k} \cdots h_{n_{p} n_{L}}^{k}\right]
$$

where $h_{i}^{k}$ is the channel gain between the $i^{\text {th }}$ basestation antenna and the $k^{\text {th }}$ user.

The $n_{U} \times n_{P} n_{L}$ channel matrix from the basestation to all the $n_{U}$ users is

$$
\mathbf{H}=\left[\begin{array}{c}
\mathbf{h}_{1} \\
\mathbf{h}_{2} \\
\vdots \\
\mathbf{h}_{n_{U}}
\end{array}\right] .
$$

The signal received on one tone by the $k^{\text {th }}$ user is

$$
r_{k}=\mathbf{h}_{k} \mathbf{x}+n_{k}
$$

where $n_{k}$ is noise received at the $k^{\text {th }}$ user and $\mathbf{x}$ is the signal vector transmitted to all users after being filtered by the basestation for the sake of transmit zeroforcing (null steering), so

$$
\mathbf{x}=\mathbf{W s}
$$

where

$$
\mathbf{W}=\left[\mathbf{w}_{1} \mathbf{w}_{2} \cdots \mathbf{w}_{n_{U}}\right]
$$


and

$$
\mathbf{s}=\left[s_{1} s_{2} \cdots s_{n_{U}}\right]^{\mathrm{T}}
$$

$s_{k}$ is the constellation symbol transmitted to the $k^{\text {th }}$ user and $\mathbf{w}_{k}$ is the $n_{P} n_{L} \times 1$ normalized filter weight for the $k^{\text {th }}$ user.

Within one symbol duration a total of $n_{U}$ symbols, one per user, are directed, from the $n_{P} n_{L}$ transmit antennas, toward the $n_{U}$ users but under the constraint that

$$
\mathbf{h}_{k} \sum_{\substack{i=1 \\(i \neq k)}}^{n_{U}} \mathbf{w}_{i} s_{i}=0
$$

For each user, $k$, Equation (5.8) means that a symbol transmitted to one user is nulled to all other users. The matrix $\mathbf{W}$ is therefore the pseudo inverse of the channel matrix $\mathbf{H}$ but after normalizing each column to maintain a constant transmitted power. The nullforming matrix $\mathbf{W}$ is then

$$
\mathbf{W}=\mathbf{H}^{H}\left(\mathbf{H H}^{H}\right)^{-1}
$$

The problem of this nullforming is that if two or more user channels are cross-correlated, then the performance of the SDMA technique will be greatly reduced. This occurs when one user comes close to the desired user, or when the scattering environment is in such a way that makes the user channels correlated. Also, a near-far problem is expected when the basestation antennas are co-located and one or more mobile users are close to the basestation while others are located far away. This creates an unbalanced channel matrix. To reduce this correlation and improve the spatial separability of the users, a decorrelation (i.e. cross-correlation reduction) algorithm is proposed in Section 5.3. 


\subsubsection{Signal Model of a Multi Antenna Mobile Users (downlink MIMO)}

We switch now to the general case when each mobile user has several antennas and downlink null steering is employed by the basestation. The frequency domain representation of the time-domain MIMO channel model can be found using equation (2.38) in section 2.4. The channel for one OFDM tone seen by the $k^{\text {th }}$ user is

$$
\mathbf{H}_{k}=\left[\begin{array}{llll}
\mathbf{H}_{1}^{k} & \mathbf{H}_{2}^{k} \cdots \mathbf{H}_{n_{p}}^{k}
\end{array}\right]
$$

where $\mathbf{H}_{p}^{k}$ is the $n_{R} \times n_{L}$ channel matrix from the $P^{t h}$ array to the $k^{\text {th }}$ user's antennas. The channel seen by the basestation to the antennas of all $n_{U}$ users' is given by the $n_{R} n_{U} \times n_{P} n_{L}$ total channel matrix

$$
\mathbf{H}=\left[\mathbf{H}_{1}^{T} \mathbf{H}_{2}^{T} \cdots \mathbf{H}_{n_{U}}^{T}\right]^{T} .
$$

Our goal is to spatially multiplex to each user as many symbols as the rank of his effective channel matrix, which can be done using eigen beamforming, but without causing any interference to other users. The signals received by all users are

$$
\mathbf{r}=\mathbf{H} \mathbf{x}+\mathbf{n}
$$

where $\mathbf{x}=\mathbf{W d}$ are the corresponding transmitted symbols, and $\mathbf{n}$ is the zero mean complex Gaussian noise vector at the receive antennas. $\mathrm{W}$ is the null steering matrix (pre-filtering matrix) used for all the users, and

$$
\mathbf{d}=\left[\begin{array}{llll}
\mathbf{d}_{1} & \mathbf{d}_{2} & \cdots & \mathbf{d}_{n_{U}}
\end{array}\right]^{T}
$$

with

$$
\mathbf{d}_{k}=\left[d_{k}^{1} d_{k}^{2} \cdots d_{k}^{n_{D}}\right]
$$

being the modulated data vector transmitted to the $k^{\text {th }}$ user, where $n_{D}$ is the number of modulation symbols transmitted to a user, that depends on his effective channel matrix in each assigned tone. 
In order for the vector $\mathbf{d}_{k}$ not to be seen by the other users, it should be rotated towards the null space of the total channel matrix of the other users, $\mathbf{H}_{k}^{\mathrm{C}}$, of size $n_{R}\left(n_{U}-1\right) \times n_{P} n_{L}$. This is the matrix between the basestation antennas and all the antennas of the other users, which is $\mathbf{H}$ after removing $\mathbf{H}_{k}$, so

$$
\mathbf{H}_{k}^{\mathrm{C}}=\left[\mathbf{H}_{1}^{T}, \cdots, \mathbf{H}_{k-1}^{T}, \mathbf{H}_{k+1}^{T}, \cdots, \mathbf{H}_{n_{U}}^{T}\right]^{T}
$$

Rotating $\mathbf{d}_{k}$ towards the null space of $\mathbf{H}_{k}^{\mathrm{C}}$ is a linear transformation achieved by expressing $\mathbf{d}_{k}$ in terms of a new coordinate system. This coordinate system is defined by the null singular vectors of $\mathbf{H}_{k}^{\mathrm{c}}$ (i.e. the singular vectors that correspond to the null singular values of $\mathbf{H}_{k}^{\mathrm{C}}$ ). By singular value decomposition of $\mathbf{H}_{k}^{\mathrm{C}}$ we get

$$
\mathbf{H}_{k}^{\mathbf{C}}=\mathbf{U}_{\mathbf{H}_{k}^{\mathrm{c}}} \Lambda_{\mathbf{H}_{k}^{\mathbf{c}}} \mathbf{V}_{\mathbf{H}_{k}^{\mathrm{c}}}^{\mathrm{c}}
$$

where $\mathbf{U}_{\mathbf{H}_{k}^{\mathrm{c}}}$ and $\mathbf{V}_{\mathbf{H}_{k}^{\mathrm{c}}}$ are $n_{R}\left(n_{U}-1\right) \times n_{R}\left(n_{U}-1\right)$ and $n_{P} n_{L} \times n_{P} n_{L}$ unitary matrices, respectively, and

$$
\Lambda_{\mathbf{H}_{k}^{\mathbf{c}}}=\operatorname{diag}\left(\sigma_{1}^{\mathbf{H}_{k}^{\mathbf{c}}}, \sigma_{2}^{\mathbf{H}_{k}^{\mathbf{c}}}, \cdots, \sigma_{n_{R}\left(n_{U}-1\right)}^{\mathbf{H}^{\mathbf{c}}}\right)
$$

where $\sigma_{i}^{\mathbf{H}_{k}^{\mathrm{c}}}$ is the $i^{\text {th }}$ singular value of $\mathbf{H}_{k}^{\mathrm{C}}$, sorted in descending order.

If $\mathbf{W}_{k}$ is the matrix whose columns are the last $n_{P} n_{L}-n_{R}\left(n_{U}-1\right)$ columns of $\mathbf{V}_{\mathbf{H}_{k}^{\mathrm{c}}}$ (the null singular vectors for the $k^{\text {th }}$ user), and

$$
\mathbf{W}=\left[\mathbf{W}_{1} \mathbf{W}_{2} \cdots \mathbf{W}_{n_{U}}\right]
$$

then, for all users,

$$
\mathbf{H}_{k}^{\mathrm{C}} \mathbf{W}_{k}=\mathbf{0}
$$

Equation (5.19) implements the zero-interference constraint which means that a signal transmitted to a user is nulled to all other users. Weighting the transmitted signals, d, 
with $\mathrm{W}$ will create a block diagonal matrix $\mathrm{HW}$, which becomes the effective channel matrix seen by the basestation to all users leading to the needed zero interference constraint. The efficiency of this null steering depends, as mentioned above, on the interuser cross correlations as well as on the eigenvalue spread of $\mathbf{H H}^{H}$. If the basestation antennas are all co-located then different physical locations of the mobile users will cause a poorly balanced $\mathbf{H}$, leading to the near-far problem. On the other hand distributing the basestation antenna arrays reduces the eigenvalue spread of $\mathbf{H H}^{H}$ resulting in more balanced power transfer gains which significantly enhances the efficiency of the null steering system.

With this signal model, the effective channel matrix seen by the $k^{\text {th }}$ user is $\mathbf{H}_{k} \mathbf{W}_{k}$, which can be used to spatially multiplex a maximum of $\operatorname{rank}\left(\mathbf{H}_{k} \mathbf{W}_{k}\right)$ of data symbols to the $k^{\text {th }}$ user. This can be done by eigen beamforming the data vector of user $k$ using singular value decomposition of $\mathbf{H}_{k} \mathbf{W}_{k}$. That is,

$$
\mathbf{H}_{k} \mathbf{W}_{k}=\mathbf{U}_{\mathbf{H}_{k} \mathbf{W}_{k}} \Lambda_{\mathbf{H}_{k} \mathbf{w}_{k}} \mathbf{V}_{\mathbf{H}_{k} \mathbf{W}_{k}}^{H}
$$

with

$$
\Lambda_{\mathbf{H}_{k} \mathbf{W}_{k}}=\operatorname{diag}\left(\sigma_{1}^{k, \mathbf{H}_{k} \mathbf{W}_{k}} \sigma_{2}^{k, \mathbf{H}_{k} \mathbf{W}_{k}} \cdots \sigma_{\operatorname{rank}\left(\mathbf{H}_{k} \mathbf{w}_{k}\right)}^{k, \mathbf{H}_{k} \mathbf{w}_{k}}\right)
$$

where $\sigma_{i}^{k, \mathbf{H}_{k} \mathbf{W}_{k}}$ is the $i^{\text {th }}$ singular value of $\mathbf{H}_{k} \mathbf{W}_{k}$ and $\left(\sigma_{i}^{k, \mathbf{H}_{k} \mathbf{W}_{k}}\right)^{2}$ is the gain of the $i^{\text {th }}$ spatial channel (eigen mode) available for the $k^{\text {th }}$ user. The effective channel model for the $k^{\text {th }}$ user is

$$
\begin{aligned}
& \hat{\mathbf{s}}_{k}=\mathbf{D}_{k}\left(\mathbf{H}_{k} \mathbf{W}_{k} \mathbf{d}_{k}+\mathbf{n}_{k}\right) . \\
& \mathbf{d}_{k}=\mathbf{M}_{k} \mathbf{s}_{k} \cdot \\
& \mathbf{s}_{k}=\left[s_{1}^{k} s_{2}^{k} \cdots s_{\operatorname{rank}\left(\mathbf{H}_{k} \mathbf{W}_{k}\right)}^{k} .\right. \\
& \hat{\mathbf{s}}_{k}=\left[\hat{s}_{1}^{k}, \hat{s}_{2}^{k}, \cdots, \hat{s}_{\operatorname{ramk}\left(\mathbf{H}_{k} \mathbf{W}_{k}\right)}^{k}\right]^{T} .
\end{aligned}
$$


The spatial multiplexing matrix, $\mathbf{M}_{k}$, is formed from the columns of $\mathbf{V}_{\mathbf{H}_{k} \mathbf{w}_{k}}$. For dominant mode transmission (directing the user's power along the largest spatial eigen mode), for example, $\mathbf{M}_{k}$ is simply the first column of $\mathbf{V}_{\mathbf{H}_{k} \mathbf{w}_{k}}$ and $\mathbf{s}_{k}=\left[s_{1}^{k}\right]$, but to transmit two symbols, $\mathbf{M}_{k}$ is formed from the first two columns of $\mathbf{V}_{\mathbf{H}_{k} \mathbf{w}_{k}}$ and $\mathbf{s}_{k}=\left[s_{1}^{k}, s_{2}^{k}\right]$, and so on, with maximum number of transmitted symbols equal to the rank of $\mathbf{H}_{k} \mathbf{W}_{k}$. The spatial de-Multiplexing matrix, $\mathbf{D}_{\mathbf{k}}$, is formed from the first columns of $\mathbf{U}_{\mathbf{H}_{k} \mathbf{W}_{k}}^{H}$, with the number of columns equal to the number of symbols transmitted in $\mathbf{s}_{k}$.

Although the near-far problem is reduced in this system due to distributing the BS antenna arrays around and close to the users, making the differences of the users' distances to the basestation in the order of hundreds of meters, the power gains of the channel matrices still differ from one user to another due to their different locations. Optimal energy allocation, for the each user's eigen mode, is therefore found through the water pouring algorithm.

The effective signal model for the $k^{\text {th }}$ user of each eigen mode after block diagonalization of the total channel matrix by $\mathbf{W}$, multiplexing and de-multiplexing using $\mathbf{M}_{k}$ and $\mathbf{D}_{k}$, and employing the water pouring algorithm on the eigenvalues of $\mathbf{H}_{k} \mathbf{W}_{k}$ is

$$
\hat{s}_{i}^{k}=\sqrt{\frac{E_{s}}{n_{P} n_{L}}} \sqrt{\vartheta_{i}^{k} \lambda_{i}^{k}} s_{i}^{k}+n_{i} .
$$

where $E_{s}$ is the amount of energy available for each tone of the OFDM symbol, $\lambda_{i}^{k}$ is the $i^{\text {th }}$ eigenvalue of $\mathbf{H}_{k} \mathbf{W}_{k}, \vartheta_{i}^{k}$ is the power share, as determined by water pouring, given to this eigen mode and $n_{i}$ is the additive Gaussian noise.

The water pouring algorithm starts with the eigen mode with the lowest gain (smallest $\lambda_{i}^{k}$ ). If that lowest gain mode does not guarantee the required SNR at the receiver, this weak spatial subchannel will be discarded and the algorithm will distribute the available 
energy between the remaining $\operatorname{rank}\left(\mathbf{H}_{k} \mathbf{W}_{k}\right)-1$ spatial subchannels. The algorithm will continue reducing the number of spatial subchannels until the required SNR is achieved in every spatial subchannel. The worst case is to leave a user with a single spatial subchannel (dominant mode transmission).

For this null steering to work, two conditions have to be fulfilled. We should have at least one null singular vector for each of the $n_{U}-1$ users and the matrices in $\mathbf{H}_{k}^{\mathrm{C}}$ have to be pair-wise uncorrelated (or, at least, have low cross-correlation). We can control the first condition by making $\mathbf{H}_{k}^{\mathrm{C}}$ a wide matrix, by increasing the number of basestation antennas compared to the total number of users' antennas. The second condition depends on the location of the users with respect to each other and on the environment around their antennas and the basestation antennas.

If the basestation antennas are co-located at the high tower of the basestation, then three problems are expected, a high pair-wise correlation between the users' channel matrices who come close to each other, an unbalanced total channel matrix due to the near-far problem and a risk of low rank and high condition number of $\mathbf{H}_{k} \mathbf{W}_{k}$. But when the basestation antennas are distributed then the users, instead of communicating with one point, will communicate with an enlarged space dimension (multi-point basestation). This reduces the negative effect of the near-far problem, but the inter-user correlation can remain high for users that are close to each other. To reduce this correlation and improve the spatial separability of the users, we present the following decorrelation algorithm.

\subsection{Decorrelation Algorithm}

In each tone of the OFDM symbol, we serve only a subset of $n_{S}$ users out of the total set of $n_{U}$ users. The subset is chosen as the users whose channel matrices for that tone have the lowest pair-wise cross-correlations. This is accomplished by the following algorithm:

- Find all possible subsets of $n_{S}$ out of $n_{U}$ users.

- Find the pair-wise cross correlation coefficient between the channel matrices of each pair $(k, l)$ of users in the subset, according to the Pearson product-moment correlation [79][80]: 


$$
\eta=\frac{\left.\sum_{m} \sum_{n} \mid\left[\mathbf{H}_{k}\right]_{m}-\overline{\mathbf{H}}_{k}\right)\left(\left[\mathbf{H}_{l}\right]_{r m}-\overline{\mathbf{H}}_{l}\right)}{\sqrt{\left.\left(\sum_{m} \sum_{n} Y\left[\mathbf{H}_{k}\right]_{m m}-\overline{\mathbf{H}}_{k}\right)^{2}\right)\left(\sum_{m} \sum_{n}\left(\left[\mathbf{H}_{l}\right]_{n m}-\overline{\mathbf{H}}_{l}\right)^{2}\right)}}
$$

where $\left[\mathbf{H}_{k}\right]_{n m}$ is $m, n^{t h}$ element of the channel matrix of user $k$ and $\overline{\mathbf{H}}_{k}$ is the arithmetic mean of the entries of $\mathbf{H}_{k}$.

- Find the mean correlation coefficient of each subset, and then choose the subset with the lowest mean.

By this strategy each user will be assigned to a different number of OFDM tones, in such a fashion that ensures spatial separation to enable the SDMA technique to work properly. The total channel matrix, $\mathbf{H}$, defined in (5.11), will be reduced to only include the $n_{S}$ users that have been assigned to that tone.

\subsection{Simulation Results and Discussion}

The system's uncoded BER performance is evaluated by means of computer simulation, according to the following system parameters. A Ricean correlated channel matrix is generated assuming a Gaussian angular spectrum with an angular spread ranging randomly between $5-30^{\circ}$ for each array, with uniformly distributed angles of departure. The Ricean $K$-factor is randomly selected between $0-10 \mathrm{~dB}$ for each array, and the Stanford University interm-3 (SUI-3) model is used for the power delay profile and path loss model given in [36] [35] (see Section 4.5). We calculated the pathloss using equation (2.1) in which the constants $P L_{0}$ and $\gamma$ are calculated based on the correction terms for frequency and antenna heights, as in [36], where we assume a carrier frequency of 2.5 $\mathrm{GHz}$, user antenna elevation of 2 metres, basestation array elevation of 10 metres, $d_{0}=$ 60 metres and terrain type B constants. The results presented here are the average over a large number of different random channel realizations. A 64-tone OFDM symbol is used.

First we simulate the downlink zeroforcing system (MISO system), described in Section 5.2.1, where each user is assumed to have a single antenna. Figure 5.1 simulates 


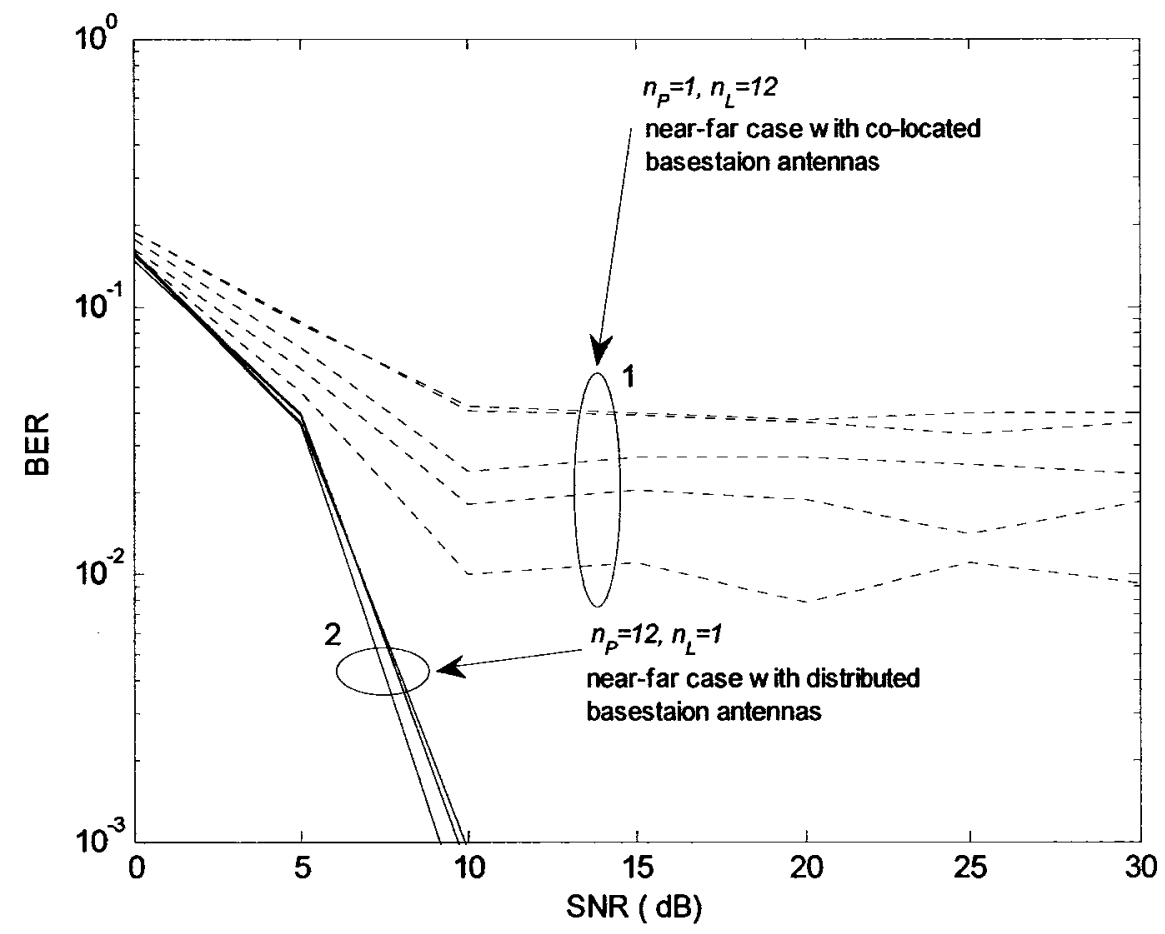

Figure 5.1. BER performance of a MISO propagation scenario, before and after the distribution of the basestation antennas, with $D_{\min }=60, D_{\max }=$ 2000 .

two propagation scenarios. The first one is when 5 users are being served by a traditional basestation that has only one antenna array composed of $n_{L}=12$ antennas. No intra-user correlation is assumed. A near-far problem is simulated here where two users are intentionally placed close to the basestation while the others are randomly placed at distances ranging between $D_{\min }=60$ and $D_{\max }=2000$ metres. Curves labeled (1) show the average uncoded-BER of each mobile user of this case. A bad performance is clear due to unbalanced channel matrix seen by the basestation to all users. Curves labeled (2) simulates the second propagation scenario in which the basestation antennas are distributed as $n_{P}=12$ arrays with one antenna each. A more balanced channel matrix is created due to random user's distances to the distributed arrays. 


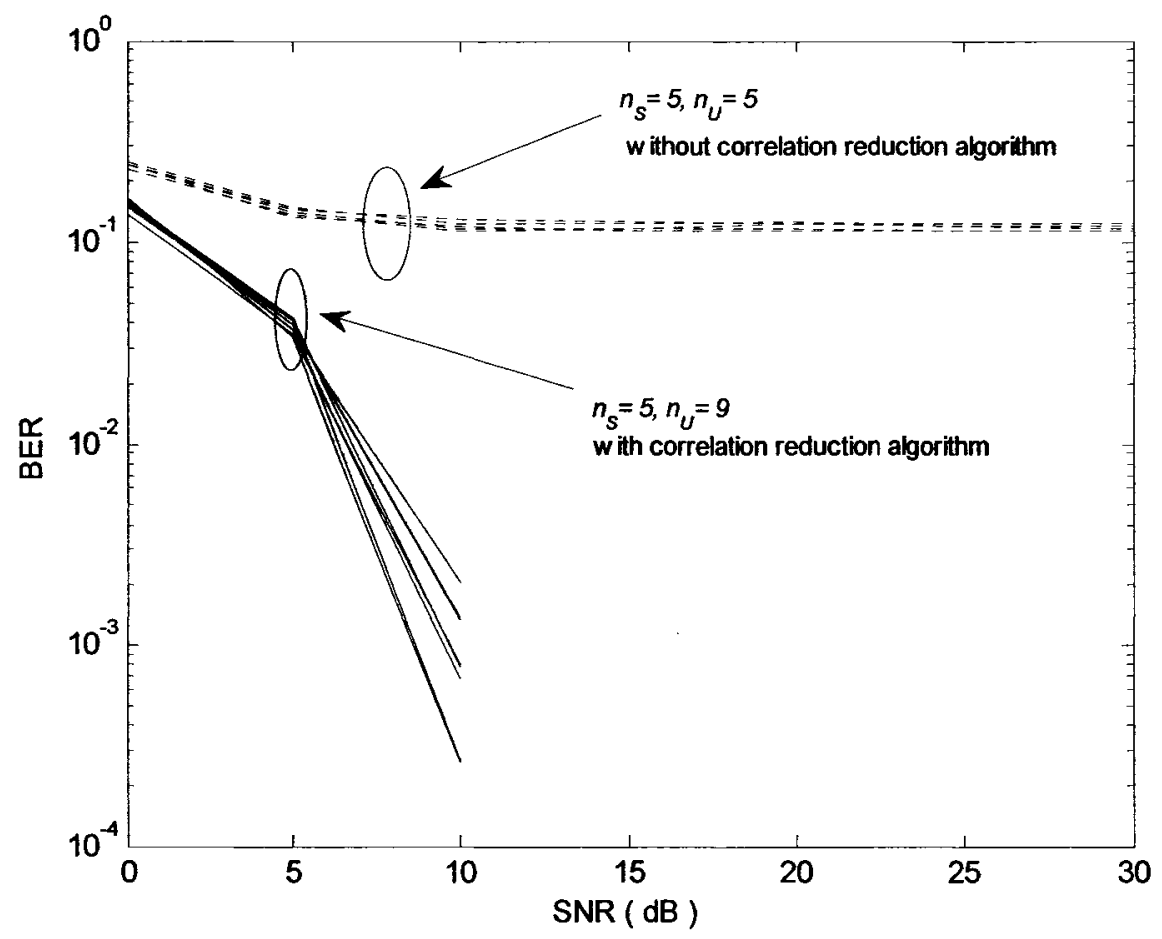

Figure 5.2. BER performance of a MISO propagation scenario, before and after the reduction of inter-user cross-correlations, with $n_{P}=12$, $n_{L}=1$

Figure 5.2depicts the case when two or more users come close to each other. We generate this scenario by making the channel vectors of those closely located users highly correlated in $25 \%$ of the OFDM tones only. No near-far scenario is assumed. Curves labeled (1) show a bad performance of this case because the basestation zeroforcing transmission system could not spatially separate the highly correlated mobile users. Curves labeled (2) shows a much improved BER performance after employing the crosscorrelation reduction algorithm which selects, in each tone, only 5 , out of 9 , users who has the lowest pair-wise cross-correlations. 
Second, we simulate the MIMO case (Section 5.2.2) where two uncoded QPSK symbols $\left(n_{D}=2\right)$ are spatially multiplexed to each user on each tone. Figure 5.3 illustrates the adverse effects of intra- and inter-user correlation on system BER performance, when the proposed correlation reduction algorithm is not employed. The basestation has 16 co-located antennas $\left(n_{P}=1, n_{L}=16\right)$, and there are $n_{U}=5$ users, each with $n_{R}=3$ antennas. All the users are served by each OFDM tone, $\left(n_{S}=5\right)$. The nominal system throughput is $2 \times n_{D} \times n_{S}=20 \mathrm{bits} / \mathrm{sec} / \mathrm{Hz}$. Curves 1 and 2 in Figure 5.3 show the average system BER in the absence of high inter-user correlation. Curve 1 corresponds to the case where there is also no high intra-user correlation, whereas Curve 2 reflects the presence of high intra-user correlation. Intra-user correlation is avoided by using a large basestation antenna separation of $\Delta_{\mathrm{T}}=10$ and assuming an angular spread of $30^{\circ}$ with Gaussian angular spectrum, and is more prominent when $\Delta_{\mathrm{T}}$ is reduced to 0.5 and the angular spread is $1-2^{\circ}$ with Laplacian angular spectrum. As can be seen, the intra-user correlation leads to a significant degradation in performance. The performance is even worse when inter-user correlation is present. Curves 3 and 4 show the uncodedBER performance when high inter-user correlation occurs, without and with intra-user correlation, respectively. In both cases the SDMA system could not compensate for the correlation, and very poor performance is realized, even at high SNR. The inter-user correlation was introduced by artificially correlating the channel matrices of users $1 \& 2$ for $25 \%$ of the OFDM tones, simulating the case when the two users are close to each other. 


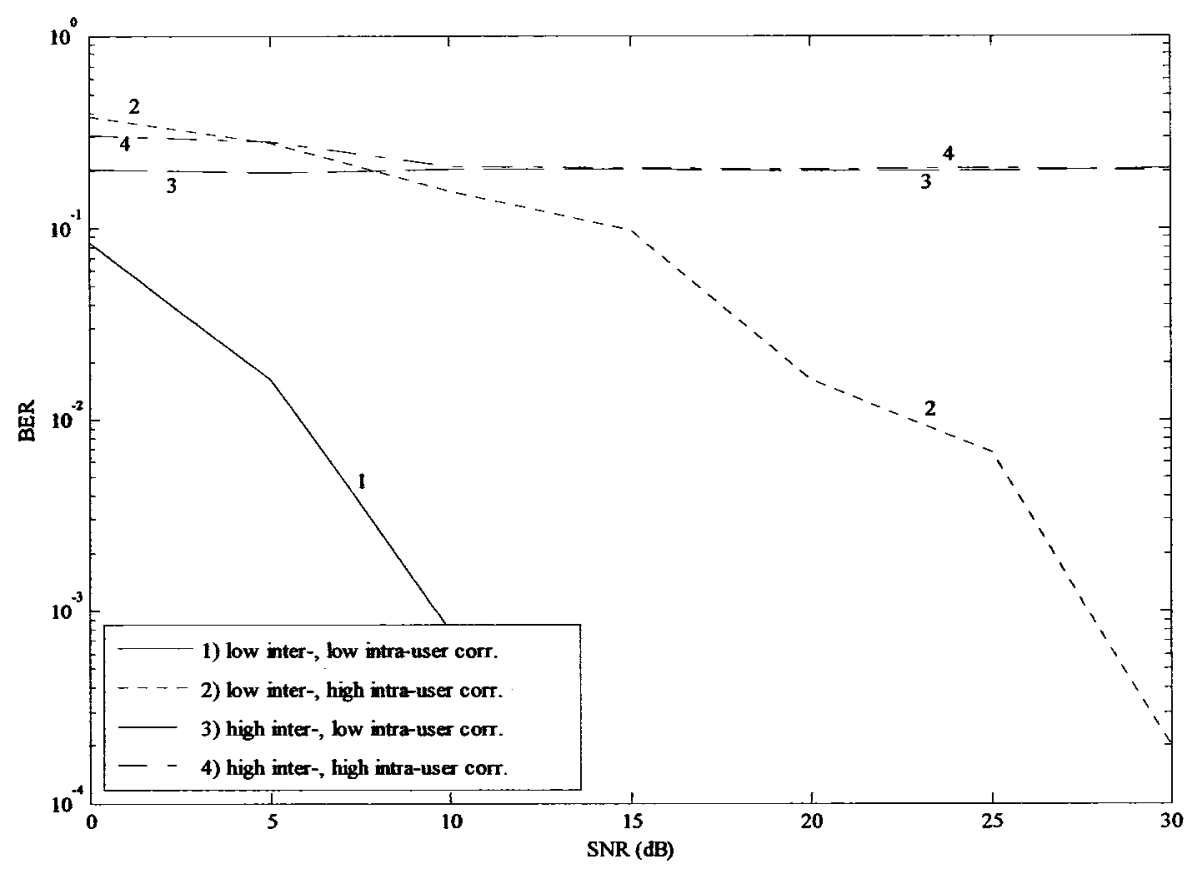

Figure 5.3. System BER performance with co-located basestation antennas and different correlation scenarios, without correlation reduction, with $n_{P}=1$, $n_{L}=16, n_{R}=3, n_{U}=5, n_{S}=5$.

As shown in Figure 5.4, distributing the basestation antennas around the cell only provides a marginal improvement in BER performance when high inter-user correlation is present(intra-user correlation is reduced by distributing the antennas). This case corresponds to $n_{P}=8$ antenna arrays of $n_{L}=2$ antennas each, with the same inter-user correlation used in the previous example. As can be seen, the BER error floor drops slightly, from 0.2 to about 0.06 , but an error floor nonetheless remains. Plotting the individual BER curves of the five users indicates that the poor average performance is due to the high BER's of the two highly correlated users (users $1 \& 2$ ). The other three users experience performance similar to the low-correlation case shown in Figure 5.3. 


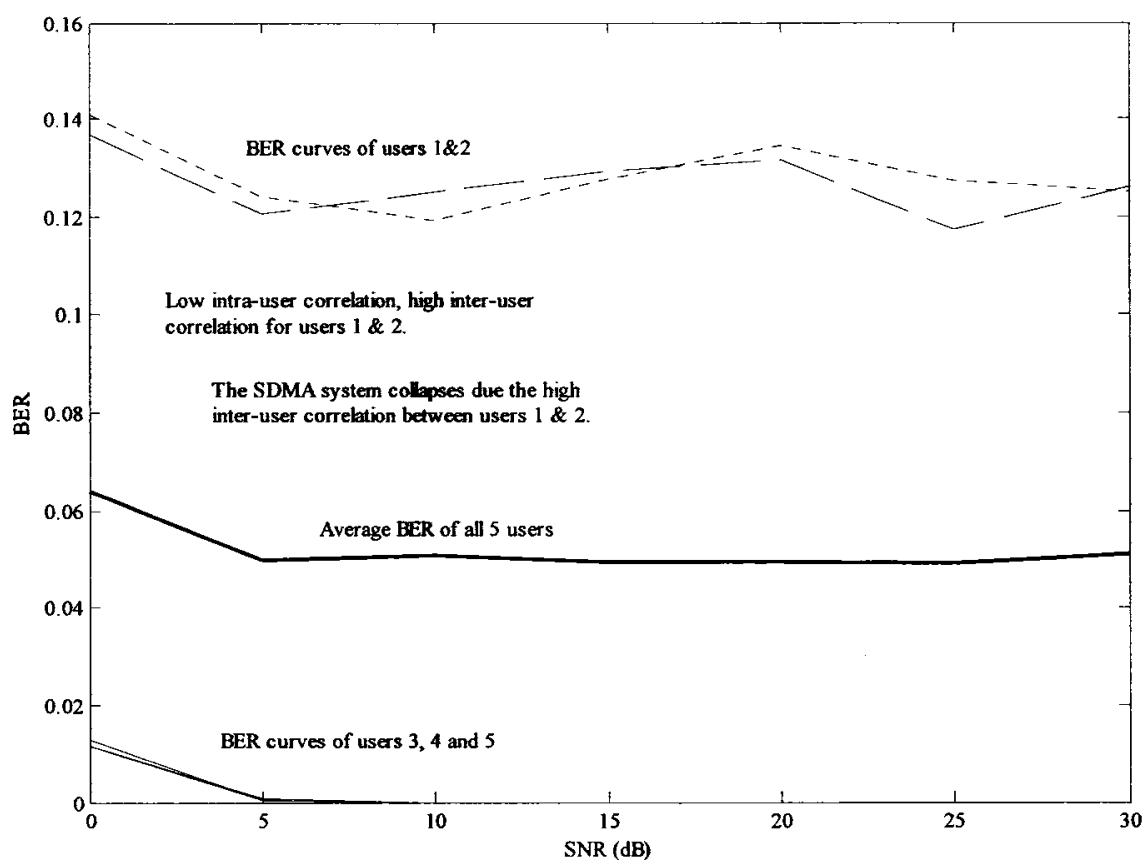

Figure 5.4. BER performance of system with distributed basestation antennas, without using cross-correlation reduction algorithm, with $n_{P}=8$, $n_{L}=2, n_{R}=3, n_{U}=5, n_{S}=5$.

The proposed decorrelation algorithm can drastically improve the BER performance for the highly correlated users. Figure 5.5 show the BER performance when the correlation reduction algorithm is used to select, out of a total of $n_{U}=9$ users, the $n_{S}=5$ users who have the lowest inter-user correlations for each tone of the OFDM symbol. A clear improvement in the BER curves is evident, as all the users, even the highly correlated ones (users $1 \& 2$ ), achieve the same good performance. Note that the nominal total system throughput is the same as in the previous examples $(20 \mathrm{bits} / \mathrm{sec} / \mathrm{Hz})$, but the per-user throughput is lower because there are more users. 


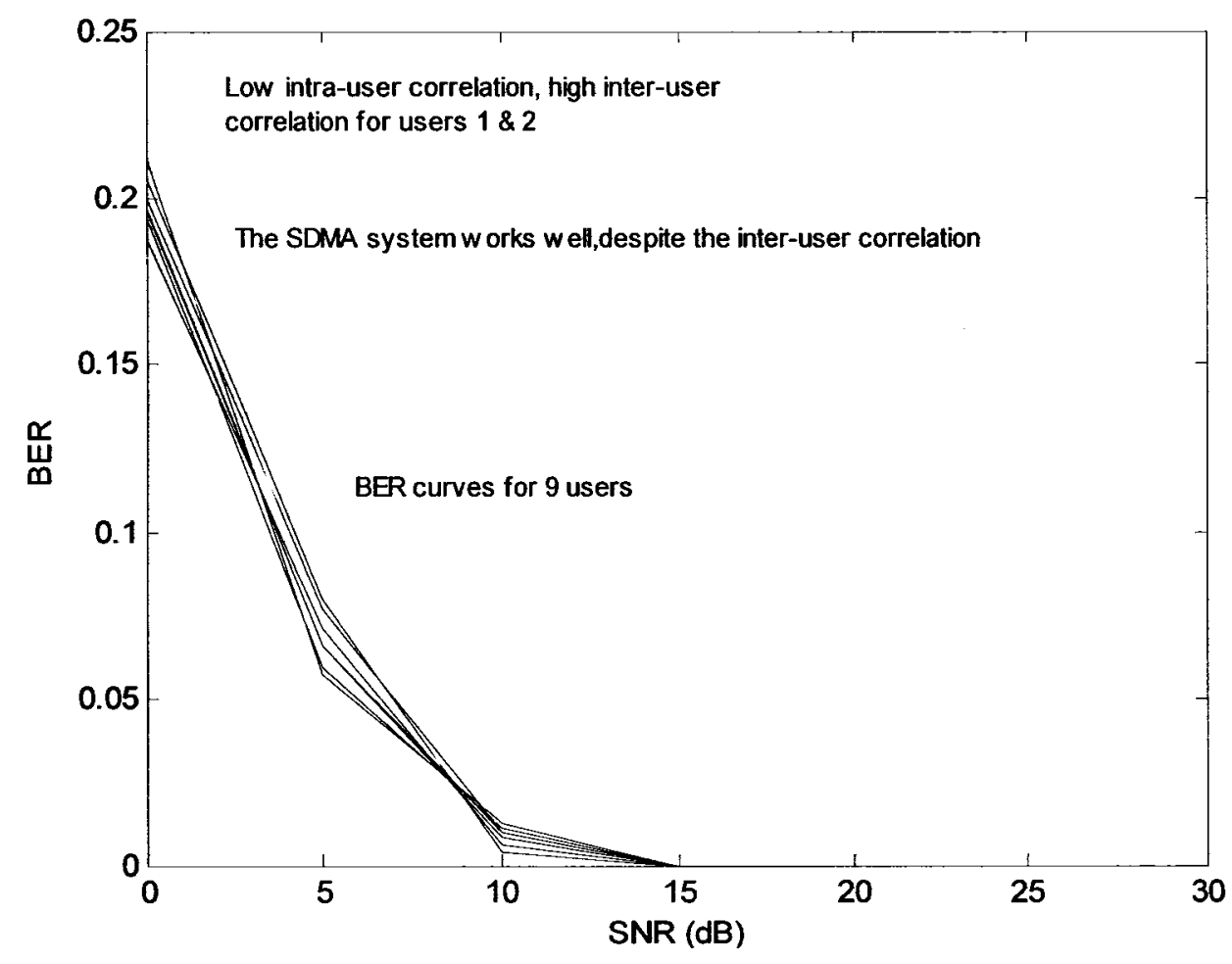

Figure 5.5. User BER performance with distributed basestation antennas, using the cross-correlation reduction algorithm, with $n_{P}=8, n_{L}=2, n_{R}=3, n_{U}=9$, $n_{S}=5$.

Figure 5.6 examines the performance of the system when the number of users who are correlated is increased. Curves $1 \& 2$ show the system performance when the correlation reduction algorithm is not used (with $n_{U}=n_{S}=4$ users), and poor system performance is evident. Curve 3 shows the case when users $1 \& 2$ and $3 \& 4$ are intercorrelated, and the correlation reduction algorithm is used to select $n_{S}=4$ out of $n_{U}=9$ users. As can be seen, much better performance is possible. The same good performance of the correlation reduction algorithm is seen in Curve 4 when three pairs of the users $(1 \& 2,3 \& 4$ and $5 \& 6)$ are inter-correlated. 


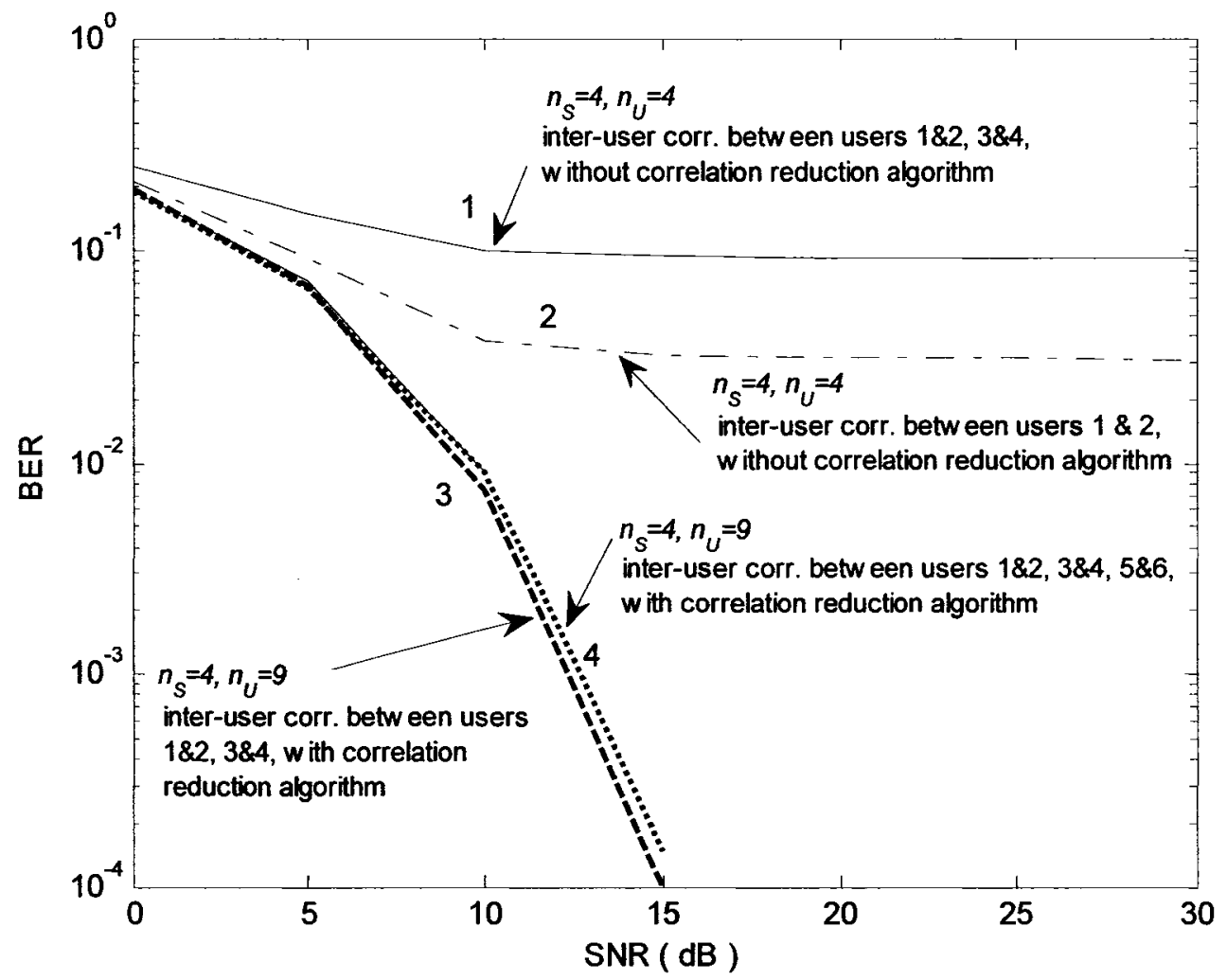

Figure 5.6. User BER performance with more correlated users, using the crosscorrelation reduction algorithm, with $n_{P}=8, n_{L}=2, n_{R}=3$.

It is worthwhile to note that the improved BER performance does not come at the expense of total system throughput, which is $16 \mathrm{bits} / \mathrm{sec} / \mathrm{Hz}$ for all curves shown in Figure 5.6, nor is it achieved by neglecting to serve the highly-correlated users. Table 5.1 shows the number of OFDM tones allocated to each user in each channel realization, for the same system parameters used with Curve 3 in Figure 5.6. The number of tones allocated to each user depends on the inter-user correlation properties (that is on the locations of users with respect to each other and on the surrounding environment), on the correlation properties of the channel matrices of each user, and on the system parameters (such as the number of tones in the OFDM symbol, the number of antennas used, the number of symbols spatially multiplexed to each user, etc). 


\begin{tabular}{|c|c|c|c|c|c|c|c|c|c|}
\hline \multirow{2}{*}{$\begin{array}{l}\text { Channel } \\
\text { realization }\end{array}$} & \multicolumn{9}{|c|}{$\begin{array}{l}\text { Number of tones allocated to each of the } 9 \text { users in each } \\
\text { channel realization }\end{array}$} \\
\hline & 7 & & 3 & 4. & 5 & 6 & 8 & 8 & 9 \\
\hline 1 & 15 & 22 & 36 & 11 & 48 & 28 & 36 & 38 & 22 \\
\hline 2 & 33 & 14 & 47 & 23 & 28 & 33 & 28 & 15 & 35 \\
\hline 3 & 36 & 20 & 30 & 13 & 29 & 18 & 40 & 36 & 34 \\
\hline$\vdots$ & $\vdots$ & $\vdots$ & $\vdots$ & $\vdots$ & $\vdots$ & $\vdots$ & $\vdots$ & $\vdots$ & $\vdots$ \\
\hline 500 & 36 & 20 & 25 & 16 & 44 & 41 & 16 & 19 & 39 \\
\hline Average & 31.0 & 20.7 & 30.8 & 21.0 & 30.7 & 30.3 & 30.6 & 30.7 & 30.2 \\
\hline
\end{tabular}

Table 5.1. Number of OFDM tones allocated to each of the 9 users (out of 64 tones), with $n_{P}=8, n_{L}=2, n_{R}=3, n_{U}=9, n_{S}=4, \mathrm{SNR}=15 \mathrm{~dB}$.

As can be seen in Table 5.1, all users are allocated at least some tones in each channel realization, although the number of tones allocated to two of the highly-correlated users is somewhat below average.

To further investigate the system fairness, Figure 5.7 shows the probability mass function of the number of tones allocated to each user for the same set of system parameters. As the figure illustrates, although not all users are allocated the same number of tones, almost all the users will be allocated a reasonable number. An acceptable degree of fairness amongst the mobile users is achieved, in the sense that accepted fairness requirements, such as the Qualcomm fairness criterion, are not violated [81]. 


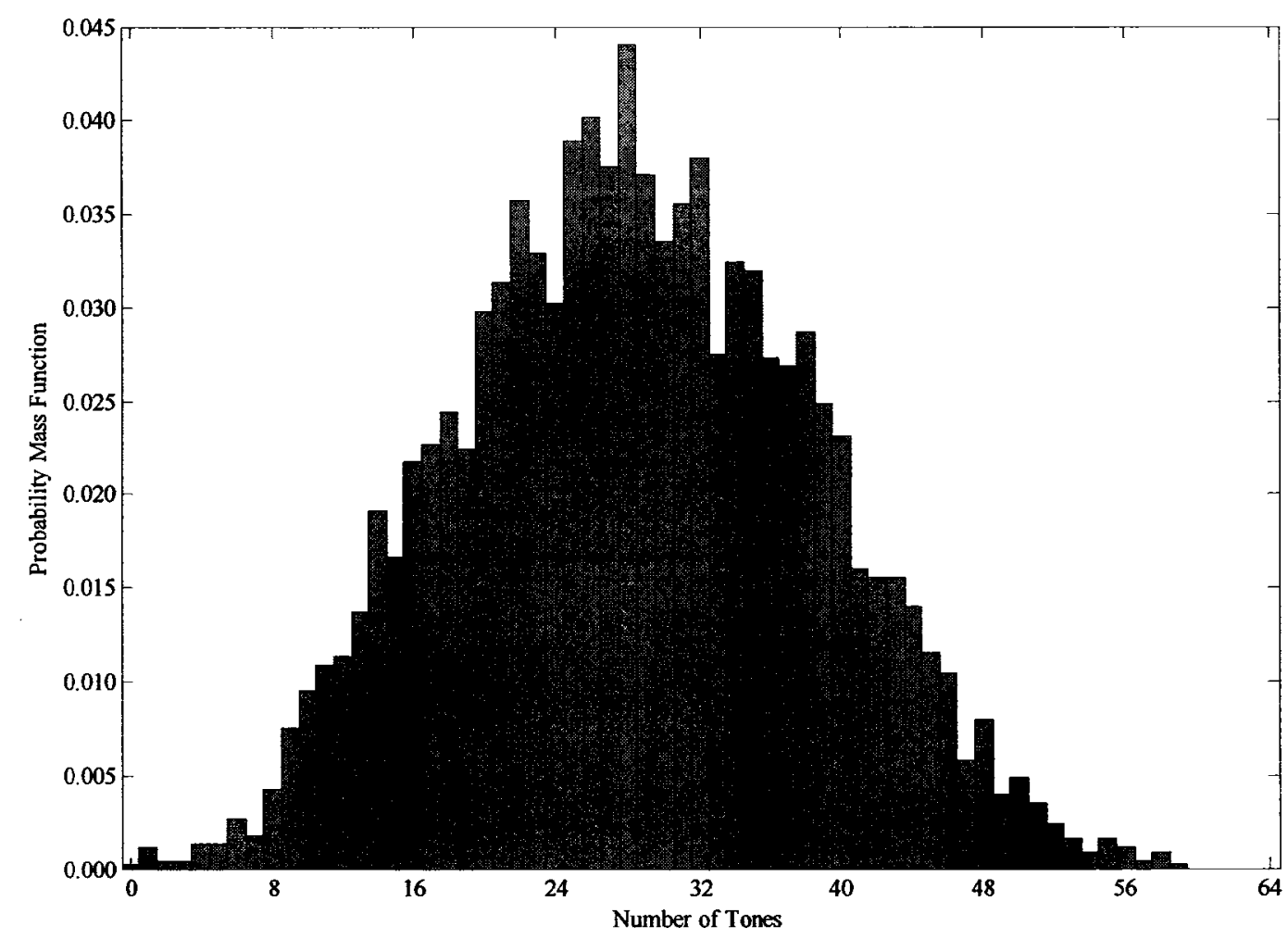

Figure 5.7. Probability mass function of the number of tones allocated to each user, with $n_{P}=8, n_{L}=2, n_{R}=3, n_{U}=9, n_{S}=4, \mathrm{SNR}=15 \mathrm{~dB}$.

Figure 5.8 illustrates the effect of different users' path losses which result in the nearfar problem. No inter-user cross-correlation is assumed, and water pouring is not used for allocation of energies to users' spatial channels. Curve 1 simulates the case when only $n_{P}=1$ basestation antenna array of $n_{L}=16$ antennas is used. The distances of the 8 users (with 2 antennas each) to the basestation array is randomly generated to range between $D_{\min }=60$ and $D_{\max }=3000$ meters. Very poor performance is observed. This is because the total channel matrix is composed of users' matrices that differ greatly in power transfer gains (greatly unbalanced). Curve 2 simulates the same case but with $n_{P}=$ 16 basestation arrays each with one antenna. Better performance is achieved because of the more balanced total channel matrix. 


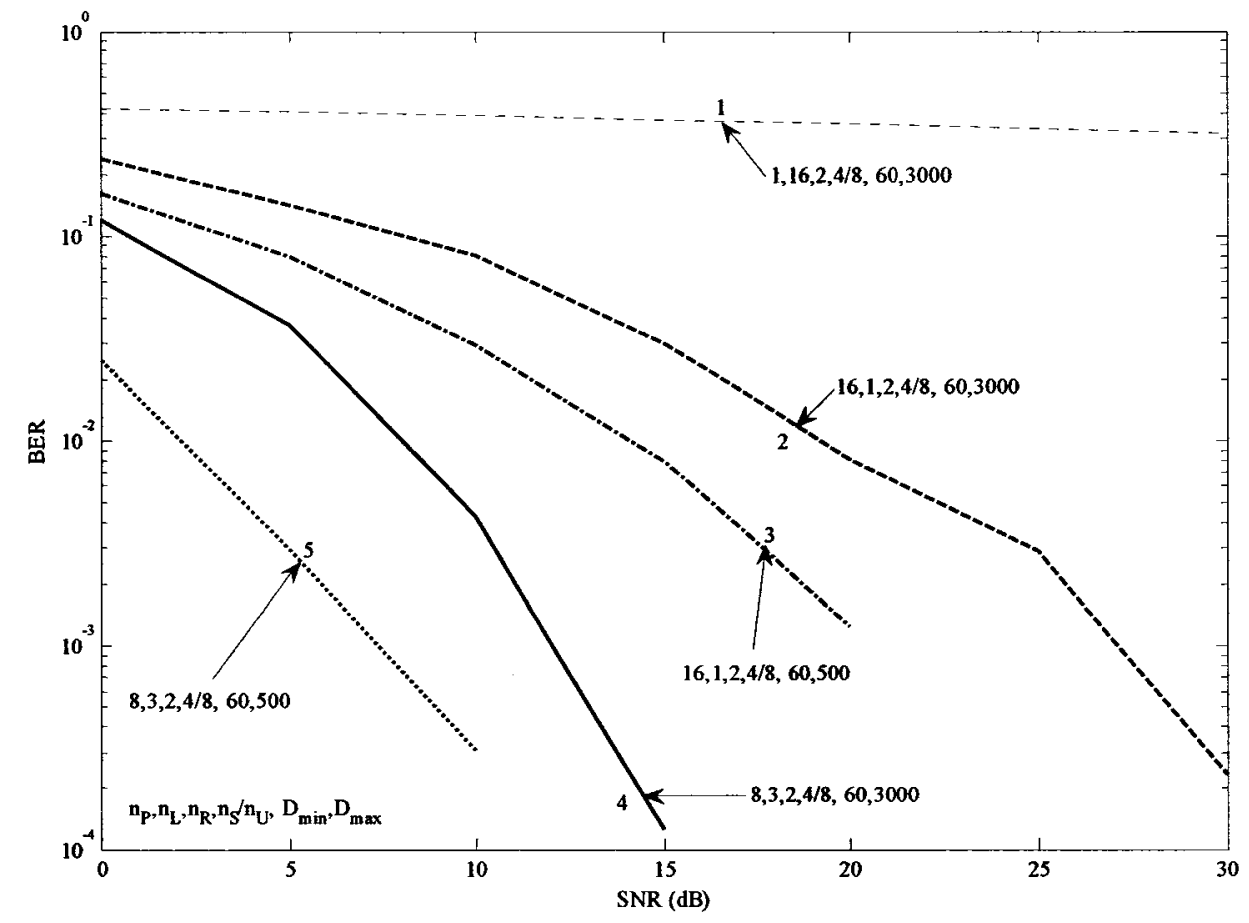

Figure 5.8. Effect of the near-far problem on the BER for different antenna configurations and different mobile users' distances to the arrays.

Even more improvement is apparent in Curve 3 because the distances to the users are made to range between 60 to 500 meters only which also results in a more balanced matrix. By increasing the number of antennas distributed around the users, as seen in Curve 4 with $n_{P}=8$ and $n_{L}=3$, the performance is further improved even when the user distances range between 60 to 3000 meters. Smaller differences of user distances with the larger number of distributed antennas results in even better performance as shown in Curve 5.

Figure 5.9 shows the effect of using the water pouring algorithm for optimal allocation of energies across the spatial channels of each user. Curve 1 depicts a propagation scenario in which 8 basestation arrays of 3 antennas each are transmitting (using null steering but without employing water pouring) to 8 users with 2 antennas each 


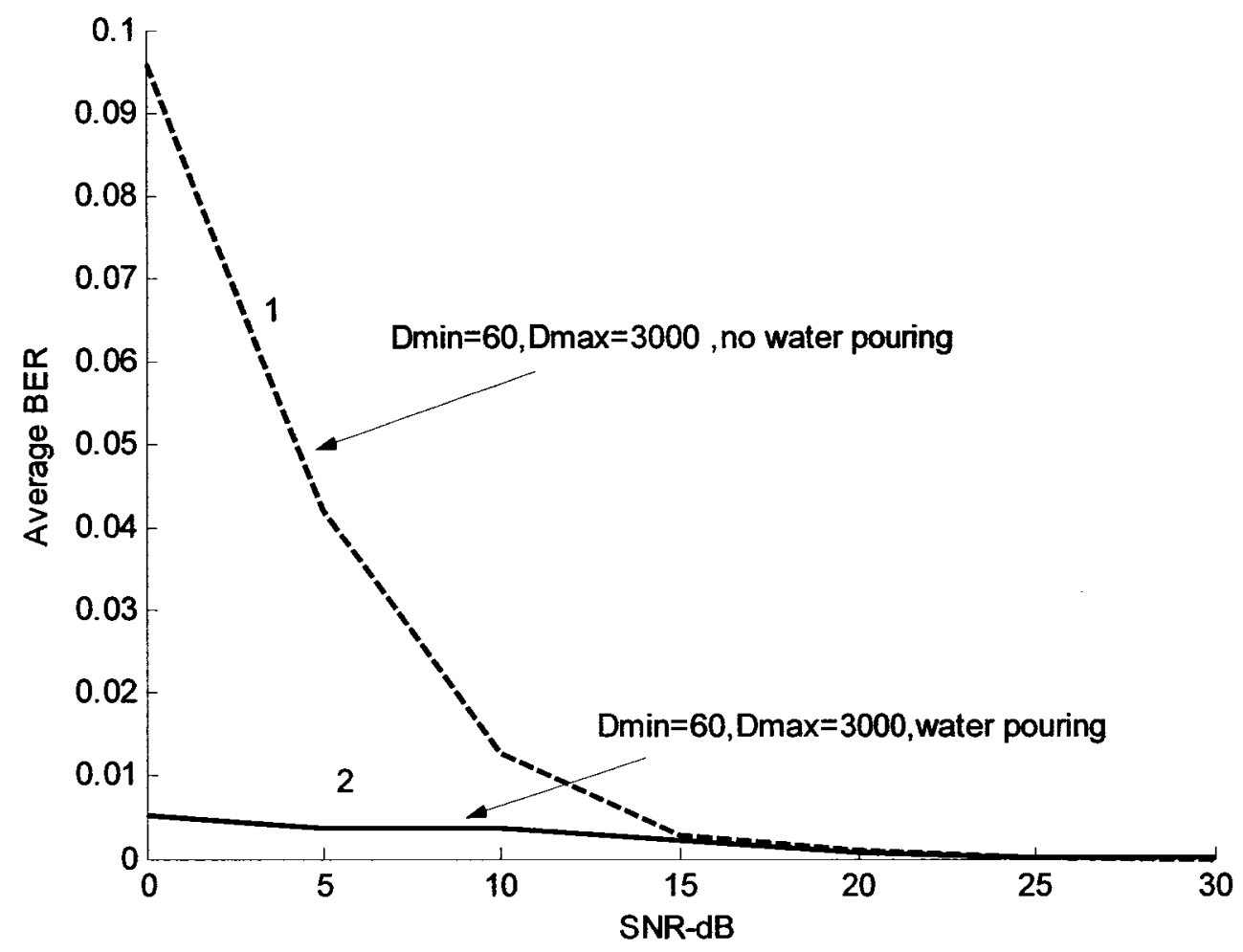

Figure 5.9. Effect of water pouring on the average $\mathrm{BER}$, with $n_{P}=8, n_{L}=3, n_{R}=2$, $n_{U}=8, n_{S}=4$.

with user pathloss distances is ranging between 60 to 3000 meters. The same propagation scenario is simulated in Curve 2 but the water pouring algorithm is used to optimally allocate energies across the available spatial channels of each user. The improvement in performance is significant at low SNR values. This is an expected result, because the capacity of the low SNR case is determined mainly by the power gain of the channel $\mathbf{H}_{k} \mathbf{W}_{k}$ so it is better to direct all power through the dominant mode, while in the high SNR case it is determined by the rank and condition number of $H_{k} \mathbf{W}_{k}$ which recommends exploiting the available spatial channels to simultaneously transmit multiple symbols to each user [34]. 


\section{Chapter 6 \\ Decorrelation of Uplink Multi-user MIMO Channels}

In this chapter we shift our focus to uplink multiuser MIMO channels. We extend the decorrelation algorithm to enhance the spatial separability of the mobile users transmitting to a base station with distributed antennas and study the role of distributing the antennas of the basestation, as multiple arrays, on such MIMO channels. We also show the advantage of the decorrelation algorithm and antenna distribution on the convergence time of LMS algorithm and on the near-far problem usually faced in multiuser channels.

\subsection{Adaptive Filters and the Eigen Structure of the Wireless Channel}

In applications where the signals and channel are time-varying, it is the rule that the system be controlled at design time. For example, in wireless channels a self designing or self adjusting filter is necessary to achieve the desired and stable system performance. This is done by adjusting a digital filter weights, according to certain criterion, each time the channel changes. These filters are called "adaptive filters.

Adaptive filters have many structures. The most widely used filter structure is the transversal filter due to its stability and analysis simplicity. In array processing a more generalized transversal filter structure, called a linear combiner structure, is used. Other structures, such as recursive and lattice, are usually used for other applications.

Adaptive algorithms can be classified into two main optimizations categories, statistical and deterministic. Least Mean Square (LMS) algorithm is usually used for the former while Recursive Least Square (RLS) algorithm is usually used for the latter case $[82][83][84][85]$.

A brief review of adaptive signal processing theory is given in this chapter followed by detailed analysis of a decorrelated uplink MIMO channel, in which an adaptive filter 
is used to spatially separate mobile users after the deployment of the previously described decorrelation algorithm to enhance the multiuser spatial separability (section 5.3).

\subsubsection{Linear Combiner Transversal Filter}

The most commonly used structure in implementing adaptive filters for antenna arrays is the linear combiner transversal structure shown in Figure 6.1. It is composed of two main parts, the filter part and the update part. The function of the filter part is to calculate the filter output $y(n)$, while the function of the update part is to adjust and optimize the set of $L$ filter weights $w_{i}$ [85]

$$
\mathbf{w}=\left[w_{0} w_{1} \cdots w_{L-1}\right]
$$

such that the output $y(n)$ becomes as close as possible to a reference signal $d(n)$.

The vector of signals, $u_{i}$, input to an adaptive filter and that might be received from an array of antennas or from different mobile users, is expressed as

$$
\mathbf{u}(n)=\left[u_{0}(n) u_{1}(n) \cdots u_{L-1}(n)\right]
$$

At each time index $n$, the filter part processes a set of input signals to produce a single output sample $y(n)$ (assuming sample per sample implementation). This output $y(n)$ is calculated as a linear combination of the input signals sampled at that time instance $n$ as

$$
y(n)=\sum_{n=0}^{L-1} w_{i}^{*} u_{i}
$$

$y(n)$ can be expressed in matrix notation as,

$$
y(n)=\mathbf{w}^{H}(n) \mathbf{u}(n)
$$

When the input signals are samples of signals transmitted from sources that are located at different positions in space, the linear combiner becomes a linear spatial filter that processes its input signals to produce the spatially filtered output $y(n)$. 


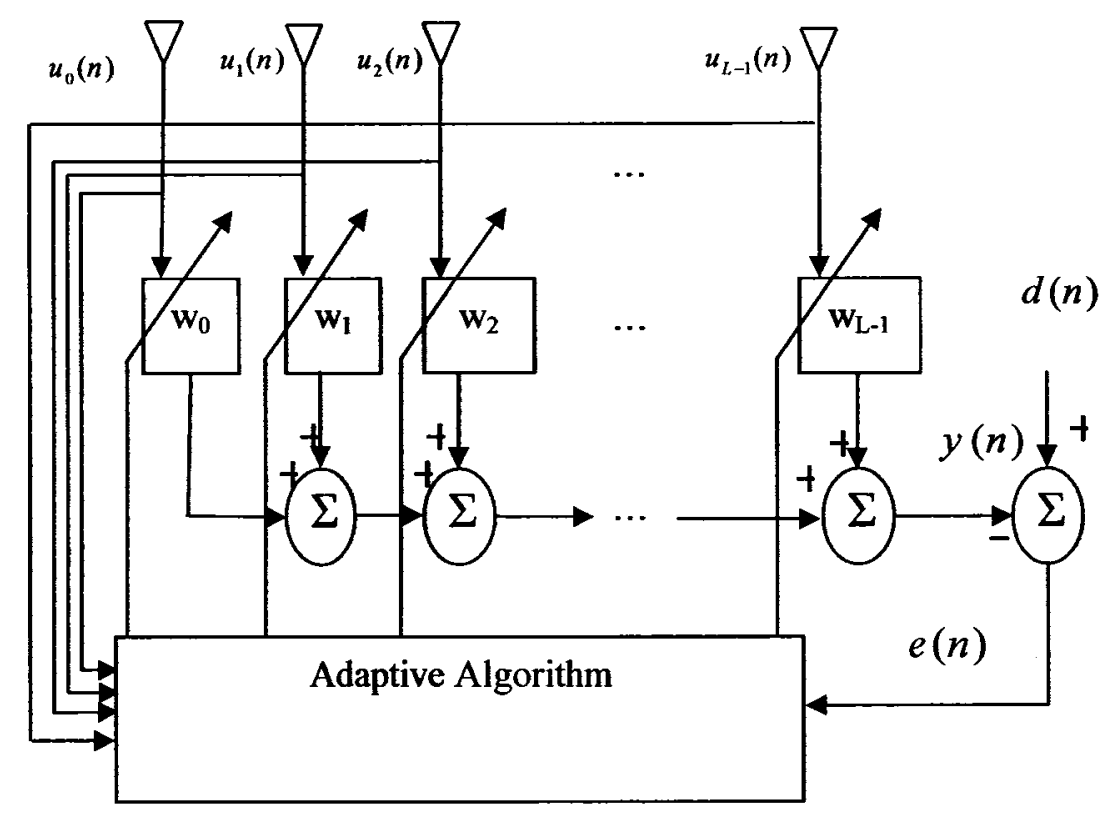

Figure 6.1. Linear combiner transversal adaptive filter.

The filter weights are calculated such that the signals received from certain source (i.e the look direction) are passed to the output while other signals received from other sources are rejected (a beamforming process) [85].

An adaptive algorithm is used, in each training interval, to adjust the filter coefficients so that a certain performance criterion is optimized in some sense. The optimization algorithm adjusts the filter weights such that the output $y(n)$ resembles a desired signal (usually arriving from the look direction) $d(n)$, or equivalently, an error signal $e(n)$ is minimized.

\subsubsection{The Least Mean Squares Problem}

Suppose the input sequence $\mathrm{u}(n)$, the desired signal $d(n)$ and the filter output are all stochastic processes then the error $e(n)$ that is defined by [82][87][88] 


$$
e(n)=d(n)-y(n)
$$

is also a stochastic process. The least mean squared error performance function is then

$$
\begin{aligned}
J_{m s} & =E\left\{e^{2}(n)\right\} \\
& =E\left\{(d(n)-y(n))^{2}\right\} \\
J_{m s} & =E\left\{d^{2}(n)\right\}-2 \operatorname{Re}\left\{\mathbf{w}^{H} \mathbf{P}\right\}+\mathbf{w}^{H} \mathbf{R} \mathbf{w}
\end{aligned}
$$

where:

$$
\mathbf{P}=E\left\{d^{*}(n) \mathbf{u}(n)\right\}
$$

is a cross-correlation vector as it measures the cross-energy of the reference signal $d(n)$ and the received signals $\mathbf{u}(n)$, and

$$
\mathbf{R}=E\left\{\mathbf{u}(n) \mathbf{u}^{H}(n)\right\}
$$

is the autocorrelation matrix.

This solution process of $\mathbf{w}$ that minimizes $J_{m s}$ leads to the normal equations

$$
\mathbf{R} \mathbf{w}_{\text {opt }}=\mathbf{P}
$$

The most straight forward approach to solving (6.10) is the direct inversion of $\mathbf{R}$ followed by the multiplication with the vector $\mathbf{P}$ leading to the well known Wiener-Hoff solution [85]

$$
\mathbf{w}_{\text {opt }}=\mathbf{R}^{-1} \mathbf{P} \text {. }
$$

For a filter of $M$ taps, the inversion requires $M^{3}$ multiplications and many other additions and subtractions. This third order complexity encourages the use of the iterative approximation solution as it is less complex. 


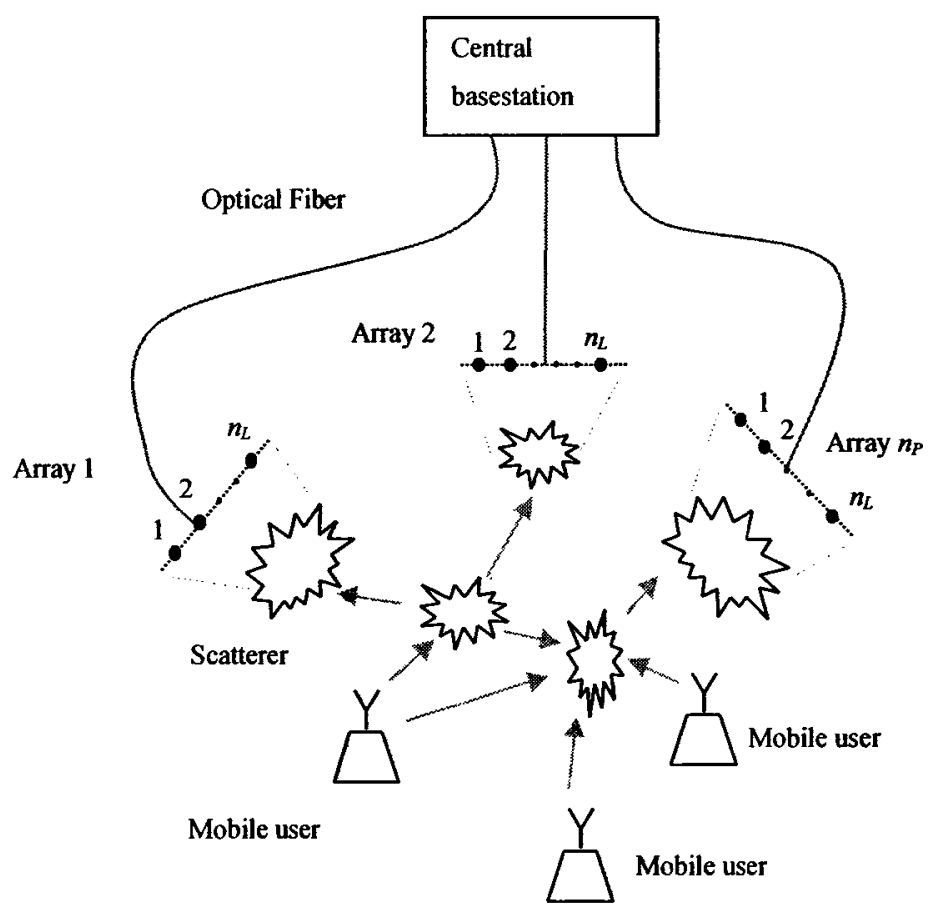

Figure 6.2. Mobile users transmitting to distributed basestation

\subsection{Decorrelation of an Adaptive Uplink Signaling Scheme}

We extend the decorrelation algorithm to improve the multi-user separability of an uplink MIMO channel (Figure 6.2). Such a reduction in channel matrix correlations decreases the convergence time of iterative adaptive algorithms such as the LMS algorithm. The channel vector of each user is assumed correlated and Ricean distributed.

Adaptive arrays at the basestation can be used to distinguish several users who have sufficiently different spatial signatures on the basestation antennas. If two or more users have correlated spatial signatures, then it is difficult for the basestation adaptive array to spatially separate them and it takes longer time to reach the optimum filter taps [85].

Because it is not possible, in the uplink transmission scenario, to coordinate between users the beamforming of their signals toward the basestation, all beamforming and signal processing has to be done at the basestation. To be able to separate users spatially, 
the basestation has to form, dynamically using adaptive signal processing, a weighting matrix that gives maximum SINR for each user. To avoid complex signal processing techniques, such as matrix inversions or RLS algorithm, we use the simple least mean square (LMS) algorithm for this purpose.

\subsubsection{Signal Model}

The distributed antenna system described in Section 2.3 is considered here. Each user will transmit a sequence of training symbols to the basestation to adjust the adaptive filter weights reach their optimum values. The vector of samples received at the basestation antennas from all users, at the $t^{\text {th }}$ tone and time instant $n$, is (Figure 6.3)

$$
\mathbf{u}^{t}(n)=\sum_{k=1}^{n_{U}} \mathbf{h}_{k}^{t} d_{k}^{t}(n)+\mathbf{v}^{t}(n)
$$

where $\mathbf{u}(n)=\left[u_{1}, u_{2}, \cdots, u_{n_{p} n_{L}}\right]$ is the vector of samples, $u_{i}$, received by basestation antennas, $\mathbf{h}_{k}^{t}$ is a quasi static channel vector, generated according to Section 2.3 , seen by the basestation, at the $t^{\text {th }}$ tone, to the $k^{\text {th }}$ user, given as

$$
\mathbf{h}_{k}^{t}=\left[\mathbf{h}_{1} \mathbf{h}_{2} \cdots \mathbf{h}_{n_{P}}\right]^{T}
$$

where $\mathbf{h}_{j}=\left[h_{1} h_{2} \cdots h_{n_{L}}\right]^{r}, h_{l}$ is the channel gain between the user's antenna and the $l^{\text {th }}$ antenna at one of the distributed arrays, $d_{k}^{t}(n)$ is the training symbol transmitted from the $k^{\text {th }}$ user and $\mathbf{v}^{k}(n)$ is the additive noise vector corrupting the received signals at time instant $n$.

To direct a distinct look direction from the basestation antennas towards each user (i.e. maximize SINR for each user), the basestation has to adaptively generate a different weight vector, $\mathbf{w}_{\mathbf{k}}$, for each user. The beamforming matrix, at the basestation, for all users can be written as

$$
\mathbf{W}=\left[\mathbf{w}_{1} \mathbf{w}_{2} \cdots \mathbf{w}_{n_{U}}\right]
$$




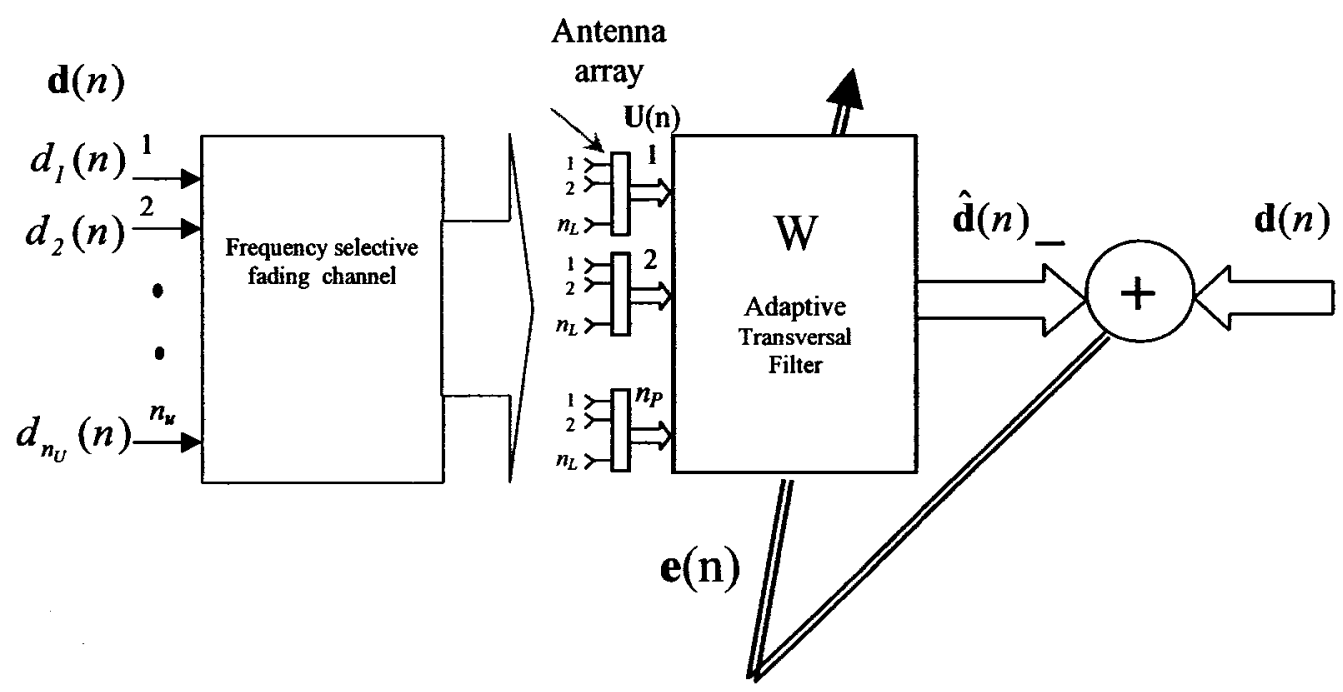

Figure 6.3. Training symbols transmitted from multiple users to a distributed basestation antennas employing adaptive filter.

The estimated $k^{\text {th }}$ user's signal, at the basestation, after using the above weight vector, is given as

$$
\hat{d}_{k}^{n}=\mathbf{w}_{k}^{H}(n) \mathbf{u}(n)
$$

The mean square error for the $k^{\text {th }}$ user is

$$
M S E=E\left\{\left(e_{k}(n)\right)^{2}\right\}=E\left\{\left(d_{k}(n)-\hat{d}_{k}(n)\right)^{2}\right\}
$$

$\mathbf{w}_{k}(n)$ is updated using the LMS algorithm (with $\mu$ as the step size) as follows [12]:

$$
\mathbf{w}_{k}(n+1)=\mathbf{w}_{k}(n)+\mu \mathbf{u}(n) e_{k}^{*}(n)
$$

The eigenvalues spread and the condition number, of the $n_{P} n_{L} \times n_{P} n_{L}$ autocorrelation matrix, $\mathbf{R}$, 


$$
\mathbf{R}=E\left(\mathbf{u}(n) \mathbf{u}^{H}(n)\right)
$$

is the decisive factor determining the speed of convergence of $\mathbf{W}(n)$ towards the optimum point on the squared error performance function. The modal decomposition of $\mathbf{R}$ is given as [84]

$$
\mathbf{R}=\mathbf{Q} \Lambda \mathbf{Q}^{H}
$$

where $\mathbf{Q}=\left[\mathbf{q}_{1} \cdots \mathbf{q}_{N}\right]$ is a unitary matrix composed of a set of orthonormal eigenvectors, $\mathbf{q}_{i}$, that correspond to the eigenvalues of $\mathbf{R}$ and that are arranged in the main diagonal of the matrix

$$
\Lambda=\operatorname{diag}\left[\lambda_{1} \lambda_{2} \cdots \lambda_{n_{U}} \cdots \lambda_{n_{P} n_{L}}\right]
$$

The first $n_{U}$ eigenvalues correspond to the $n_{U}$ mobile users while the other eigenvalues are zeros (due to a large number, $n_{P} n_{L}$, of basestation antennas). The spread of the eigenvalues is increased by the near-far problem and the correlations within the channel matrix. Distributing the basestation antennas will reduce the gain imbalance of the channel matrix and also reduce the intra-user correlations. To reduce the inter-user correlations we use the decorrelation algorithm, previously proposed in Section 5.3.

\subsection{Decorrelation of a Space-time Block Coded Uplink Signaling Scheme}

We investigate the decorrelation algorithm and the distribution of the basestation antennas on the performance of another uplink propagation scheme where multi-users, with 2 antennas each, are transmitting Alamouti space-time block codes to the basestation. 


\subsubsection{Signal Model}

On the first symbol period, user $k$ will transmit signals $s_{1}^{k}$ and $s_{2}^{k}$ from his antennas to the basestation and then transmit signals $-s_{2}^{* k}$ and $s_{1}^{* k}$ on the second symbol period. The signal model for a single user over two time periods is:

$$
\mathbf{Y}_{\mathbf{k}}=\mathbf{H}_{k} \mathbf{S}_{k}+\mathbf{n}
$$

where:

$$
\begin{aligned}
& \mathbf{S}_{\mathbf{k}}=\left[\begin{array}{cc}
s_{1}^{k} & -s_{2}^{* k} \\
s_{2}^{k} & s_{1}^{* k}
\end{array}\right] \\
& \mathbf{Y}_{\mathbf{k}}=\left[\begin{array}{ll}
\mathbf{y}_{1}^{k} & \mathbf{y}_{2}^{k}
\end{array}\right]
\end{aligned}
$$

$\mathrm{y}_{1}^{k}$ is a $n_{P} n_{L} \mathrm{x} 1$ vector of the signals received at the basestation antennas due to the symbols transmitted from the $k^{\text {th }}$ user at the $i^{\text {th }}$ symbol period.

$$
\begin{aligned}
& \mathbf{H}_{\boldsymbol{k}}=\left[\begin{array}{ll}
\mathbf{h}_{1}^{k} & \mathbf{h}_{2}^{k}
\end{array}\right] \\
& \mathbf{h}_{i}^{\boldsymbol{k}}=\left[\begin{array}{c}
\mathbf{h}_{1}^{i, k} \\
\mathbf{h}_{2}^{i, k} \\
\vdots \\
\mathbf{h}_{n_{p}}^{i, k}
\end{array}\right] \\
& \mathbf{h}_{p}^{i, k}=\left[\begin{array}{llll}
h_{1, p}^{i, k} & h_{2, p}^{i, k} & \cdots & h_{n_{L}, p}^{i, k}
\end{array}\right]^{T}
\end{aligned}
$$

where $\mathbf{h}_{p}^{i, k}$ is the $n_{L} \times 1$ vector of channel gains from the $j^{\text {th }}$ antenna of the $k^{\text {th }}$ user to the $p^{\text {th }}$ array.

$$
\begin{aligned}
& \mathbf{y}_{1}^{k}=\mathbf{h}_{1}^{k} s_{1}^{k}+\mathbf{h}_{2}^{k} s_{2}^{k} \\
& \mathbf{y}_{2}^{* k}=\mathbf{h}_{2}^{*^{k}} s_{1}^{k}-\mathbf{h}_{1}^{* k} s_{2}^{k} .
\end{aligned}
$$


The effective signal model becomes:

$$
\tilde{\mathbf{Y}}_{\mathbf{k}}=\tilde{\mathbf{H}}_{k} \mathbf{s}_{k}+\mathbf{n}
$$

where:

$$
\begin{aligned}
& \tilde{\mathbf{Y}}_{\mathbf{k}}=\left[\begin{array}{l}
\mathbf{y}_{1}^{k} \\
\mathbf{y}_{2}^{* k}
\end{array}\right] \\
& \mathbf{s}_{k}=\left[\begin{array}{l}
s_{1}^{k} \\
s_{2}^{k}
\end{array}\right] \\
& \tilde{\mathbf{H}}_{k}=\left[\begin{array}{cc}
\mathbf{h}_{1}^{k} & \mathbf{h}_{2}^{k} \\
\mathbf{h}_{2}^{* k} & -\mathbf{h}_{1}^{* k}
\end{array}\right] .
\end{aligned}
$$

When Alamouti coded signals of multiple users are spatially multiplexed, the received signal at the basestation from the $k^{\text {th }}$ user is:

$$
\tilde{\mathbf{Y}}_{\mathbf{k}}=\tilde{\mathbf{H}}_{k} \mathbf{s}_{k}+\sum_{\substack{j=1 \\ j \neq k}}^{n_{U}} \tilde{\mathbf{H}}_{j} \mathbf{s}_{j}+\mathbf{n}
$$

The estimated signal of the $k^{\text {th }}$ user is:

$$
\widehat{\mathbf{s}}_{\mathbf{k}}=\tilde{\mathbf{H}}_{k}^{H} \tilde{\mathbf{Y}}_{\mathbf{k}}
$$

The less correlated the matrices $\tilde{\mathbf{H}}_{k}$, the better the ability of the basestation to separate the multi-user's signals. We select the less correlated mobile users, in each tone, based on the decorrelation algorithm previously described in Section 5.3. 


\subsection{Simulation Results}

We present below the simulation results of both uplink signaling schemes, the adaptive signaling scheme and the space-time coded signaling scheme. We used a 32 tone OFDM symbol each loaded with a QPSK signal.

\subsubsection{Simulation Results of the Adaptive Signaling Scheme}

Figure 6.4 depicts the near-far problem where we assume a number of $n_{L}=32$ co-located $\left(n_{P}=1\right)$ basestation antennas are mounted on the top of the basestation tower. We also assume that $n_{U}=n_{S}=5$ mobile users are present in the microcell where two of them are located at a near distance around 70 metres and the other three users are located at a far distance around 1000 metres. We generate the channel according to the model given in Section 2.3 with no inter-user correlations. This makes the eigenvalue distribution of the autocorrelation matrix in (6.18) only affected by the power balance within the channel matrix. Figure 6.4 shows the $\mathrm{CDF}$ of eigenvalues and condition number of this scenario where we see how spread the eigenvalues are and how large the condition number is because of the severe near-far case.

Figure 6.5 shows a propagation scenario where we distribute the basestation antennas as $n_{P}=8$ arrays of $n_{L}=4$ antennas each. The CDF curves labeled 1 show this case where we see a significant decrease, compared to Figure 6.4, in the eigenvalue spread and a reduction in the condition number. Curves labeled 2 show more improvement (reduction in eigenvalue spread and condition number) when the number of the distributed arrays is increased to $n_{P}=16$ of $n_{L}=2$ antennas each.

In Figure 6.6 we ignore the near-far problem, by assuming all users have equal distance to the basestation, and focus on the effect of the decorrelation algorithm and distributing the basestation antennas on the performance in an environment of intra-user and inter-user correlations. Curves labeled 1 depicts the case when one array $\left(n_{P}=1\right)$ of $n_{L}=16$ co-located antennas. Here we generated correlated channel vectors, for each user, assuming Laplacian angular spectrum, 2-3 degrees angular spread and random angles of 

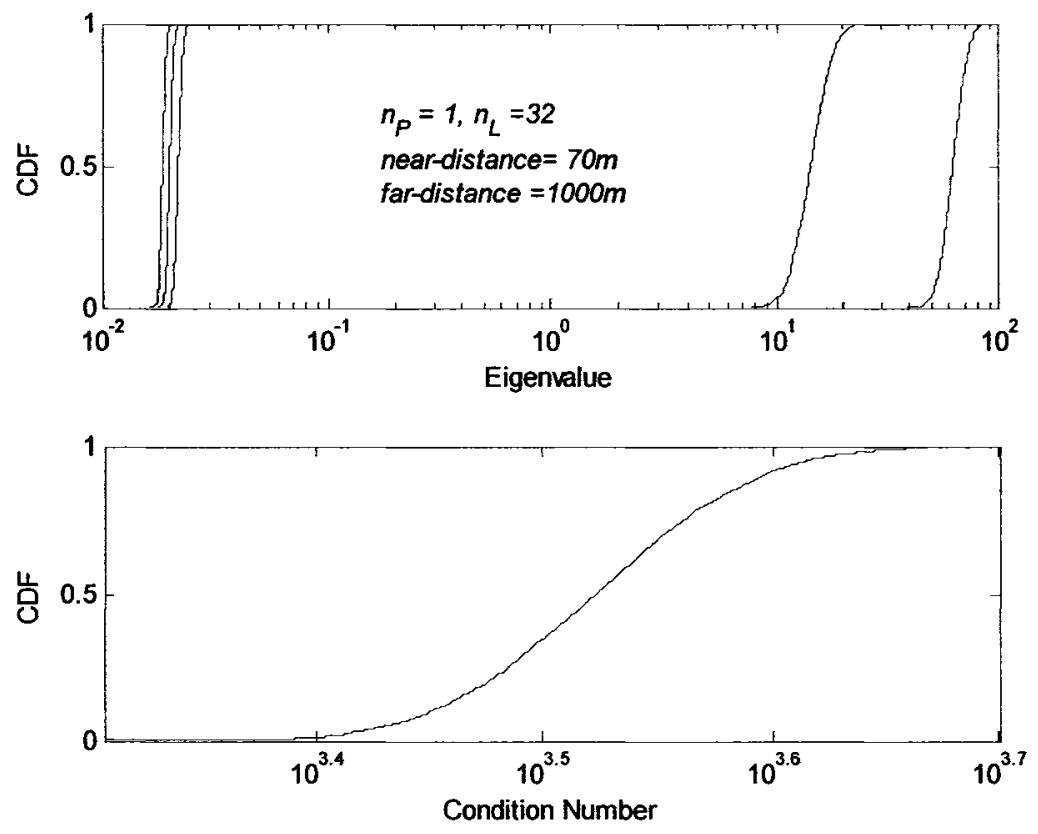

Figure 6.4. CDF of eigenvalues and condition number with near-far problem and co-located basestation antennas as $n_{P}=1, n_{L}=32, \mathrm{D}_{\min }=70 \mathrm{~m}$, $\mathrm{D}_{\max }=1000 \mathrm{~m}$.

arrival. Slow and bad convergence is clear. A much better performance is shown in the curves labeled 2, where we distributed the basestation antennas into $n_{P}=8$ arrays of $n_{L}=$ 2 antennas each. The best performance is shown in the Curves labeled 3 where we employed the decorrelation algorithm to reduce the inter-user correlation.

Figure 6.7 shows the case when $n_{S}=2$ out of $n_{U}=5$ users are highly correlated in $25 \%$ of the OFDM tones. We see that the MSE curves of those two users, labeled 1 , do not converge. Figure 6.8 shows the same previous case but we selected $n_{S}=5$ users out of $n_{U}=9$ users with the lowest inter-user correlations using the decorrelation algorithm. A much better performance is evident. 

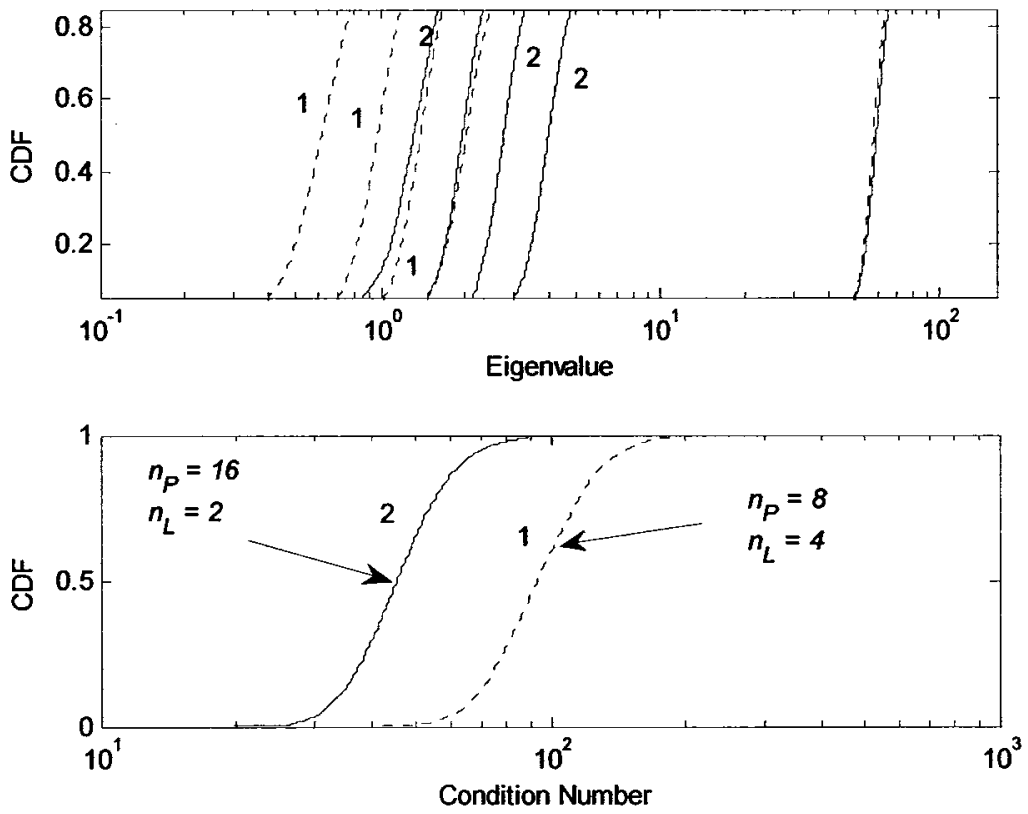

Figure 6.5. CDF of eigenvalues and condition number for with a near-far problem and distributed antennas as $n_{P}=8, n_{L}=4$, and $n_{P}=16, n_{L}=2$. 


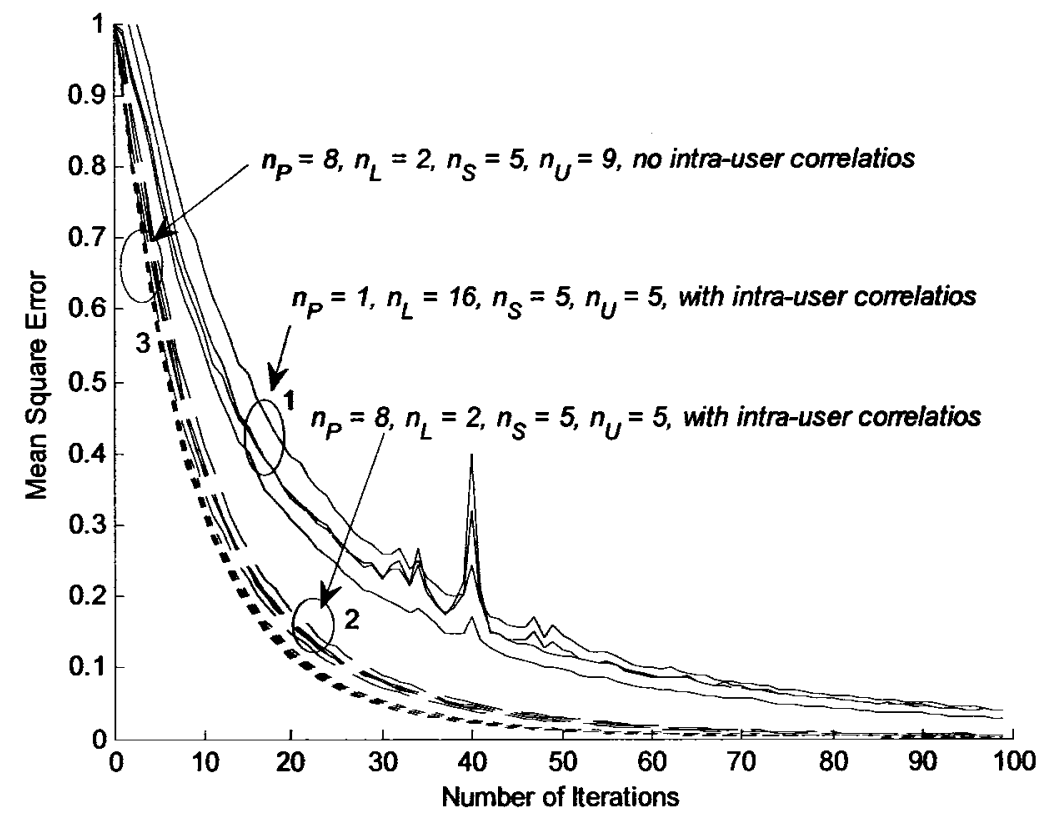

Figure 6.6. Effect of antenna configuration on the MSE of several mobile users with intra-user correlations. 


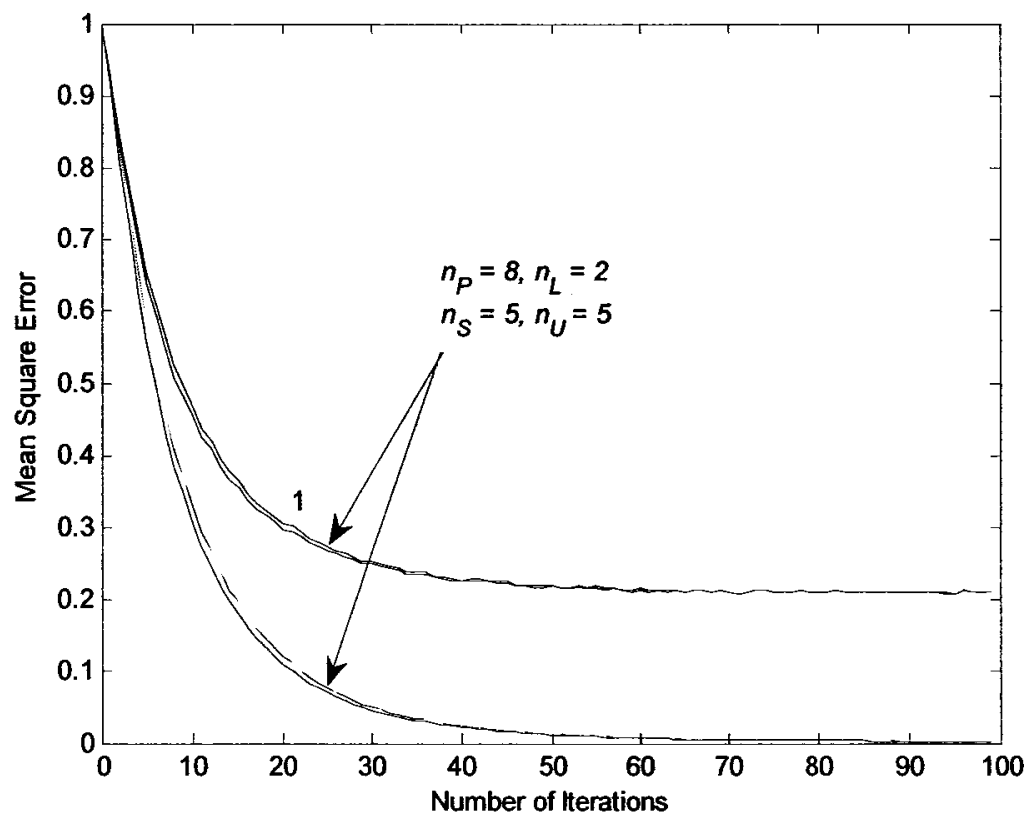

Figure 6.7. MSE of $n_{U}=5$ users with inter-user correlations without using decorrelation algorithm. 


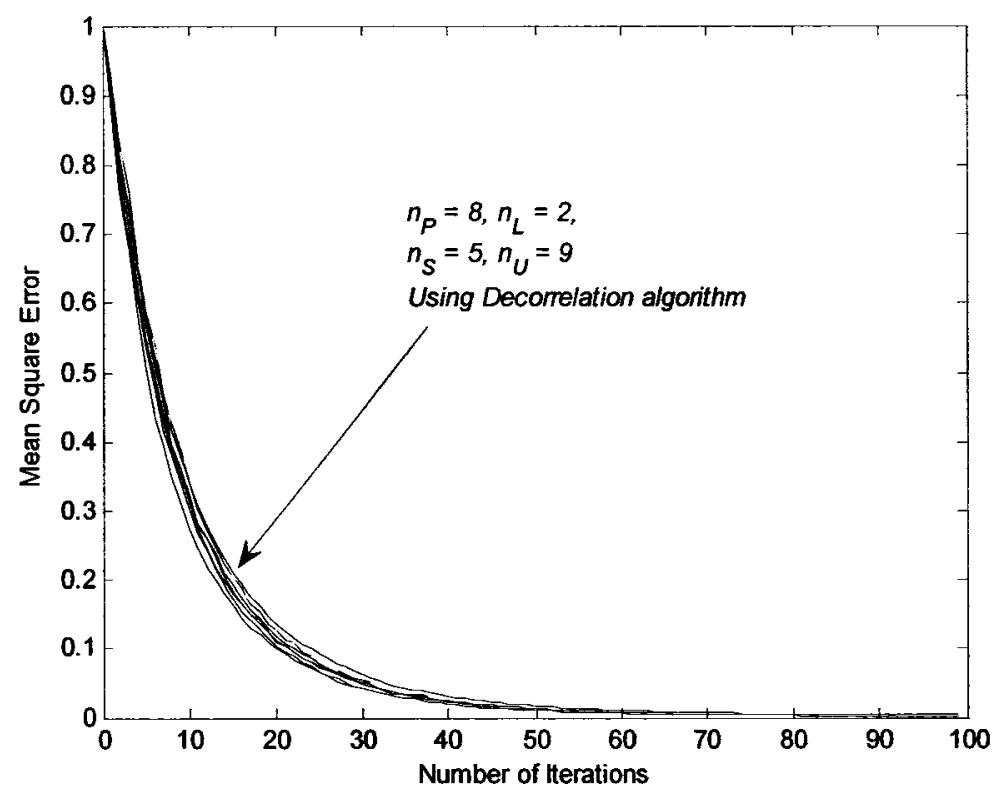

Figure 6.8. $\mathrm{MSE}$ of $n_{U}=9$ users with inter-user correlations when selecting $n_{S}=5$ users, in each OFDM tone, using the decorrelation algorithm.

\subsubsection{Simulation Results of the Space-time Block Coded Signaling Scheme}

A 64 tone OFDM symbol is used each loaded with a QPSK signal. A Ricean-correlated user channel matrix is generated for each array. We assume that all users have equal distances to the antenna arrays.

Figure 6.9 depicts the performance of a single user scenario. Curve 1 shows the case when 4 basestation antennas are co-located in one array, the $K$ factor is zero, the angular spectrum is assumed Laplacian while the angular spread is assumed 4 degrees at the arrays and 20 degrees at the user antennas. Improvement in performance is recognized in Curve 2 when distributing the antennas as 4 arrays of one antenna each. More 
improvement is shown in Curve 3 when distributing more antennas where it is assumed that the basestation has 3 arrays of 2 antennas each and that the angular spectrum is uniformly distributed with angular spread ranges randomly between 10 and 30 degrees for each array in each channel realization. These improvements are due to the reduction in intra-user correlations because of distributing the antennas of the basestation as arrays.

Figure 6.10 shows the performance when mobile users are spatially multiplexing the Alamouti coded signals to the basestation. Curve 1 shows the case when the basestation has 3 arrays with 8 antennas each $\left(n_{p}=3, n_{L}=8\right)$ and two users are spatially multiplexing the Alamouti coded signals to the basestation. No correlation reduction or user selection is employed. Improvement is recognized in Curve 2, compared to Curve 1, due to distributing more arrays $\left(n_{p}=8, n_{L}=3\right)$ while keeping the same total number of basestation antennas (i.e 24 antennas).

Curve 3 shows the case with the same antenna configuration as curve 1 but here it is assumed that 5 mobile users are present in the cell and we employ the correlation reduction algorithm to select 2 of them $\left(n_{p}=3, n_{L}=8, n_{U}=5, n_{S}=2\right)$. Significant improvement is noticed compared to Curves 1 and 2 due to reduction in inter-user correlations. As shown in Curve 4, distributing more antenna arrays of the basestation ( $\left.n_{p}=8, n_{L}=3, n_{U}=5, n_{S}=2\right)$ and employing the same selection algorithm (selecting 2 out of 5 users) as above, outperforms all previous cases. This is due to the reduction in intra-user correlations (by distributing the arrays around the users), and reduction in interuser correlations (by employing correlation reduction algorithm) which led to more spatial separation of the mobile users.

Curves $5\left(n_{p}=8, n_{L}=3, n_{S} / n_{U}=3 / 5\right)$ and $6\left(n_{p}=12, n_{L}=4, n_{S} / n_{U}=3 / 5\right)$ show the case when selecting 3 mobile users out of 5 . Bad performance is recognized even with reduction in intra-user (distributing more arrays) and reduction in inter-user correlations. This is due to the large inter-user interference which dominates the performance even with increased SNR. 


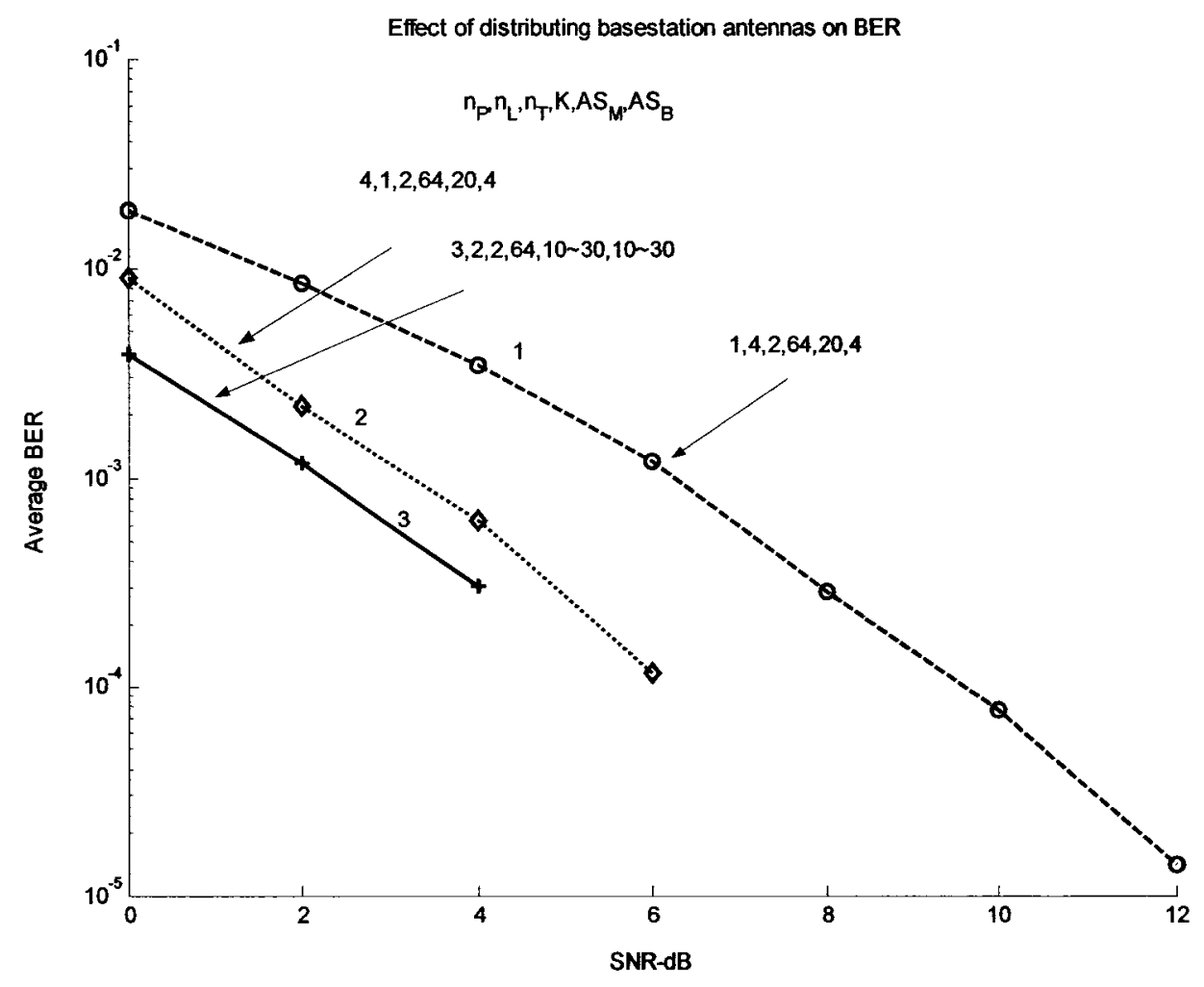

Figure 6.9. Effect of distributing the basestation antennas on the system performance 


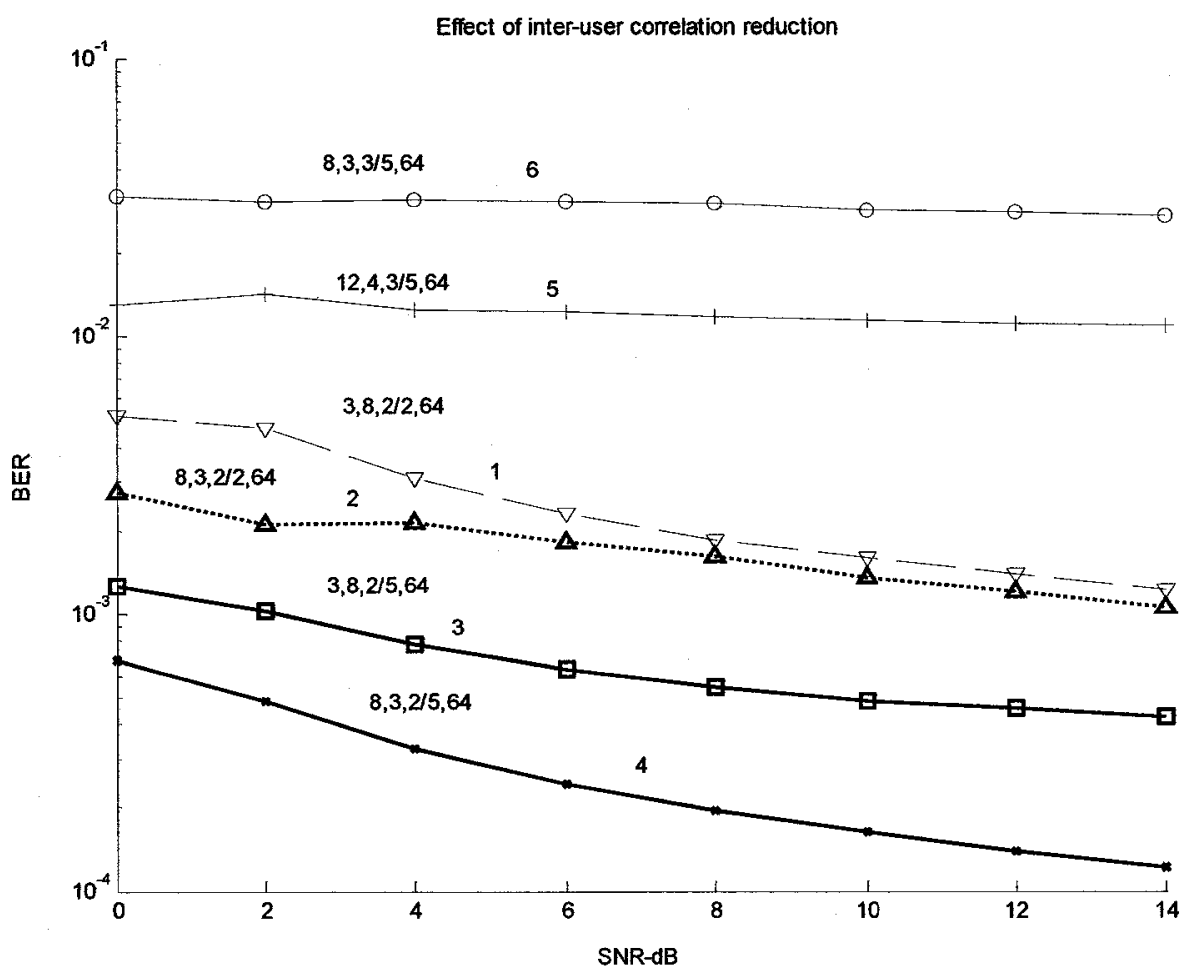

Figure 6.10. Effect of inter-user correlation reduction on average bit error rate. 


\section{Chapter 7 \\ Conclusions and Future Work}

The main motive behind employing MIMO systems in the fourth generation broadband wireless is their ability to transmit several data symbols, in each channel use, simultaneously and on the same frequency band. This makes MIMO communication systems the future of wireless systems as it offers tremendous increase in data rates with high quality and reliability. This increase doesn't require additional power or bandwidth. It is because by having multiple antennas at both ends of the wireless link, several independent spatial subchannels are opened between the transmitter and the receiver through which several data symbols can be spatially multiplexed to the receiving end. These spatial subchannels can be used to serve either multiple users in a space division multiple access (SDMA) system or/and a single user in a time division multiple access (TDMA) system.

However, correlations within the channel matrix (in both TDMA and SDMA systems) diminish the degree of independence between these subchannels which in turn heavily reduce channel capacity and system performance. Another obstacle in the way of widely implementing SDMA systems is the near-far problem which unbalances the channel matrix and increases the eigenvalue spread and condition number of the channel matrix. This situation greatly degrades system performance.

In this thesis, we considered the above two problems. We developed several decorrelation algorithms that aim at improving MIMO channel capacity. We proposed to have the basestation antennas distributed, as arrays of several antennas each, into different sites and placed at low heights within the microcell. We modeled a frequency selective MIMO channel with Ricean distributed correlated channel matrix entries (according to the angles of departure / arrival, angular spectrum and angular spread at each array) and which takes into account the line of sight, shadowing and pathloss to each array's site. 
First, in a single user propagation scenario, assuming perfect channel knowledge at the transmitter and receiver, and a Ricean frequency selective-fading channel, we developed selection algorithms both in space dimension (distributed antennas) and in frequency dimension (OFDM tones) that aim at improving the system performance by reducing MIMO channel matrix correlations (intra-user correlation), increasing spatial diversity and reducing system complexity.

Next, in a downlink multiuser (each with several antennas) propagation scenario, we developed a decorrelation algorithm that improved the spatial separability of the mobile users by reducing the inter-user correlations (a correlation of the channel matrix of a user to that of another user). Distributing the arrays around the users also balances the channel matrix leading to significant reduction in the effect of the near-far problem. Several data symbols can be spatially multiplexed to each user over each assigned OFDM tone (water filling is used to determine the relative power of each symbol) with high reliability and with good system throughput.

Next, in an uplink multiuser (each with a single antenna) propagation scenario, we extended the decorrelation algorithm to enhance an uplink SDMA signaling scheme in which the basestation, with distributed-adaptive antenna arrays, employed the decorrelation algorithm to, besides improved spatial separation of mobile users, reduced the training time needed for the convergence of the filter weights (using an iterative LMS adaptive algorithm).

Finally, we developed an enhanced uplink multiuser (each with two antennas) propagation scenario in which the selected users, based on the decorrelation algorithm, transmit Alamouti space-time codewords to the distributed basestation antennas.

\section{Suggestions for Future Research:}

- In practice, the channel and its parameters (such as antenna correlations) cannot be estimated perfectly. The performance of the decorrelation algorithms and the SDMA signaling schemes need to be characterized with channel estimation errors. 
- In this thesis, we have assumed the optical fiber link to be error and interference free. In practice however, the optical fiber link is a non-linear channel with limited dynamic range and since the OFDM signal has a large peak to average power ratio (PAPR), the dynamic range will be even more limited. A proper PAPR reduction technique of the OFDM signal and appropriate equalization technique of the optical fiber link need to be applied to the distributed antenna system and investigated.

- The number of tones allocated to users depends on the correlation situation of each user's channel matrix. It is interesting to find techniques that guarantees a constant requested data rates for a user or group of users based on the decorrelation algorithm.

- The performance of a degenerate MIMO channel (a key-hole MIMO channel) or a MIMO channel with cross-polarized antennas with a distributed basestation antennas is worth investigating.

- Finally, the deployment of the decorrelation algorithms on systems employing space-time trellis codes, dirty paper coding, and other space-time signaling schemes is an interesting research problem. 


\section{Appendix A \\ Beamforming and SDMA}

\section{A.1 Classifications of Antenna Arrays}

Antennas may be classified as isotropic (omni-directional), directional, phased array and adaptive antennas. An isotropic antenna is an antenna which has equal gain in all directions, while a directional antenna is an antenna which has more gain in certain directions (boresight directions) and less gain in others. Usually the antenna gain is measured with respect to the isotropic antenna. For example a directional antenna of 10 dBi gain means the gain of this antenna is 10 times more in the boresight direction compared to the isotropic antenna for the same input power. Passive antennas are considered reciprocal meaning that the power pattern is valid for both transmission as well as reception [89].

Composed of several antennas, phased array antenna combines the signals received on these antennas to produce an array output (Figure A.1). The phases of the received signals are adjusted such that the signals due to a source, in a certain direction, are combined in phase and this increases the gain of the antenna towards the desired source. In adaptive antennas, not only the phase but also the gain of the induced signals is dynamically adjusted to increase the gain of the antenna towards the desired direction. This adaptation is controlled by the system [90].

In downlink SDMA signaling schemes, the basestation encodes the signals transmitted to all users such that each user obtains the desired SINR. By assuming the CSI to be known to both the transmitter and the receiver, several transmit schemes have been proposed in the literature such as the transmit zero-forcing, MMSE and dirty paper coding (DPC) [91][78]. Next, we briefly go through different types of beamformers as a brief introduction to different SDMA techniques. 


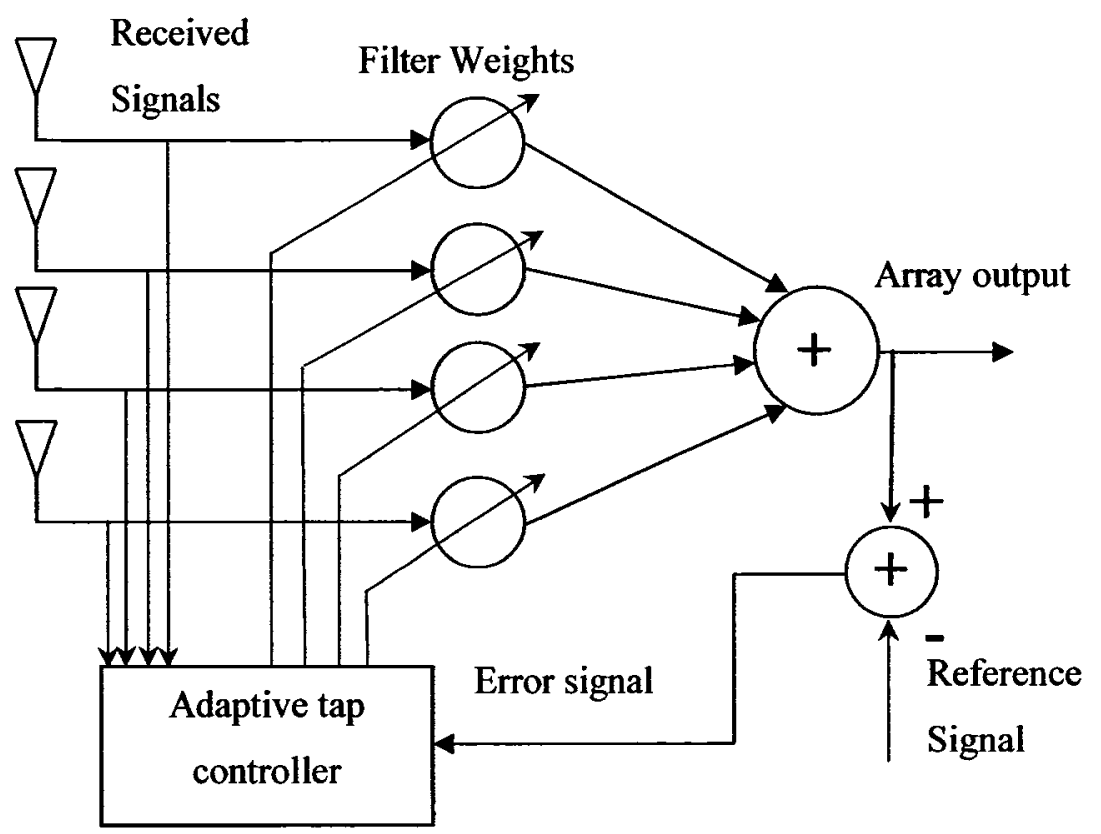

Figure A.1. Block diagram of a narrow-band adaptive beamformer antenna.

\section{A.2 Antenna Beamformers}

The array pattern or beam pattern is an indication of the power received by the array from a particular direction due to a source located at that direction. Traditionally, beamforming is defined as the process of maximizing the array beam pattern in a particular direction. In MIMO systems, it is highly desirable for the signal(s) that are transmitted from a certain antenna array (source) to be received from all directions. We would like to have a channel with a rich scattering environment that generates large angular spreads at both the transmitting and the receiving antenna arrays. In such a channel, several spatial data pipes are opened between the transmitter and the receiver. These pipes are not necessarily located in the area between the transmitting and receiving arrays. Some of theses pipes might be "connected" to the array, either receiving or/and transmitting, from behind. It is because of the scatterers around the arrays that reflect the signals in many directions that 
the fading of these signals is independent creating the spatial data pipes and increasing the rank of the channel matrix.

The beam pattern can be steered (rotated), into different directions, by mechanical rotation of the array. More effective and quick steering can be achieved electronically by adjusting the phases of the signals received on different elements to point the beam into a desired direction. This direction is some times called the look direction. The beam pattern can also be nulled (made of low value or almost zero) in other direction. The number of antenna elements determines the amount of beamforming flexibility. The beam pattern of a single-element array cannot be changed while the pattern of a two element array can be adjusted by changing the complex weight of one antenna relative to the other antenna to place one maxima (or minima) in a certain direction. With three-element antenna array, two positions on the beam pattern can be adjusted. With $n_{T}$ antenna elements $n_{T}-1$ positions can be controlled such as placing a maxima towards a desired target and $n_{T}-2$ minims (nulls) towards $n_{T}-2$ undesired targets. So with $n_{T}$ antenna array we have an array of a so called $n_{T}-1$ degrees of freedom [92].

In a multiuser channel, each mobile source will induce a steering vector (i.e., array response vector or a spatial signature) on the basestation array. Signals received from (or transmitted to) undesired sources can be cancelled by exploiting the flexibility of the antenna array weighting. The cancellation of one interfering signal by placing one null in the pattern uses one degree of freedom of the array (see Figure A.2). A null in an antenna pattern denotes a zero response. In practice, however, that is seldom achievable, and one creates a pattern with a reduced response toward undesirable interferers. Formation of nulls in the antenna pattern toward co-channel mobiles helps to reduce the co-channel interference. In the downlink, less energy is transmitted from the basestation toward these mobiles, reducing the interference from the basestation to them. In the uplink, this helps to reduce the contribution from these mobiles at the basestation [92][93]. 


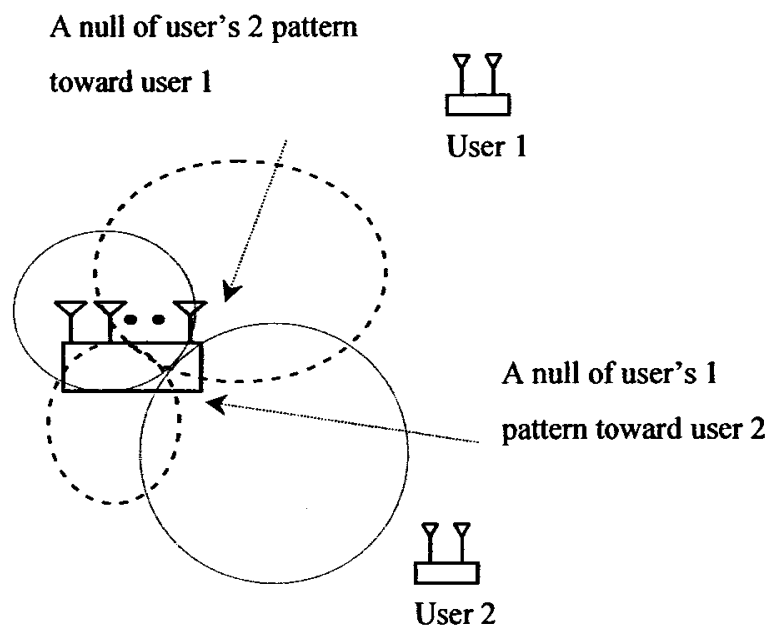

Figure A.2. A propagation scenario of a base-station system employing an SDMA technique to serve many mobiles using nullsteering.

\section{A.2.1 Traditional Beamformer}

The traditional beamformer is a delay-and-sum beamformer where all weights are of equal magnitude but the phases are selected to steer the array towards a desired direction (the look direction). The array weight vector, $\mathbf{w}$, is given by

$$
\mathbf{w}=\frac{1}{n_{R}} \mathbf{e}_{0}
$$

where $\mathbf{e}_{0}$ is the antenna array steering vector (or unit spatial signature) and $n_{R}$ is the number of antenna elements within the array. This receiver, using electronic steering, acts just like steering the array mechanically in the look direction. 
Figure A.3 shows the delay-and-sum beamformer for the case of two receiving antennas $\left(n_{R}=2\right)$ separated by a distance $D$. If the wavefront is impinging the array at angle $\theta$ and induces the signal $r_{1}(t)$ on the first element then after time

$$
T=\frac{D \cos \theta}{c}
$$

it will induce the signal $r_{2}(t-T)$ on the second antenna element. If we delay the signal on the first by $T$ seconds, then the two signals are in-phase and can be added constructively. This beamformer enhances the SNR in the absence of any interferer source and when the noise signals, picked up at the antennas, are uncorrelated. If the noise vector is $\mathbf{n}=\sigma_{n}^{2} \mathbf{I}$ then the output noise power is:

$$
\begin{aligned}
p_{n} & =\mathbf{w}^{H} \mathbf{n w} \\
& =\frac{\sigma_{n}^{2}}{n_{R}}
\end{aligned} .
$$

We conclude that the noise power output from the array is reduced $n_{R}$ times which makes the array gain to be $n_{R}$. In the presence of an interferer $s_{I}$ (operating on the same frequency band), the output from the array due to this interferer is

$$
\mathbf{w}^{H} \mathbf{s}_{I}=\frac{\mathbf{s}_{0}^{H}}{n_{R}} \mathbf{s}_{I} .
$$

We see that the desired signal is jammed by the interference resulting in reduced SINR.

\section{A.2.2 Null-steering Beamformer}

The null-steering beamformer intends at canceling the signals arriving from (or transmitted to) undesired mobile users. It produces nulls (a zero response) in the array manifold (response pattern) toward unwanted mobile users. One scheme [94] achieves null steering by estimating 


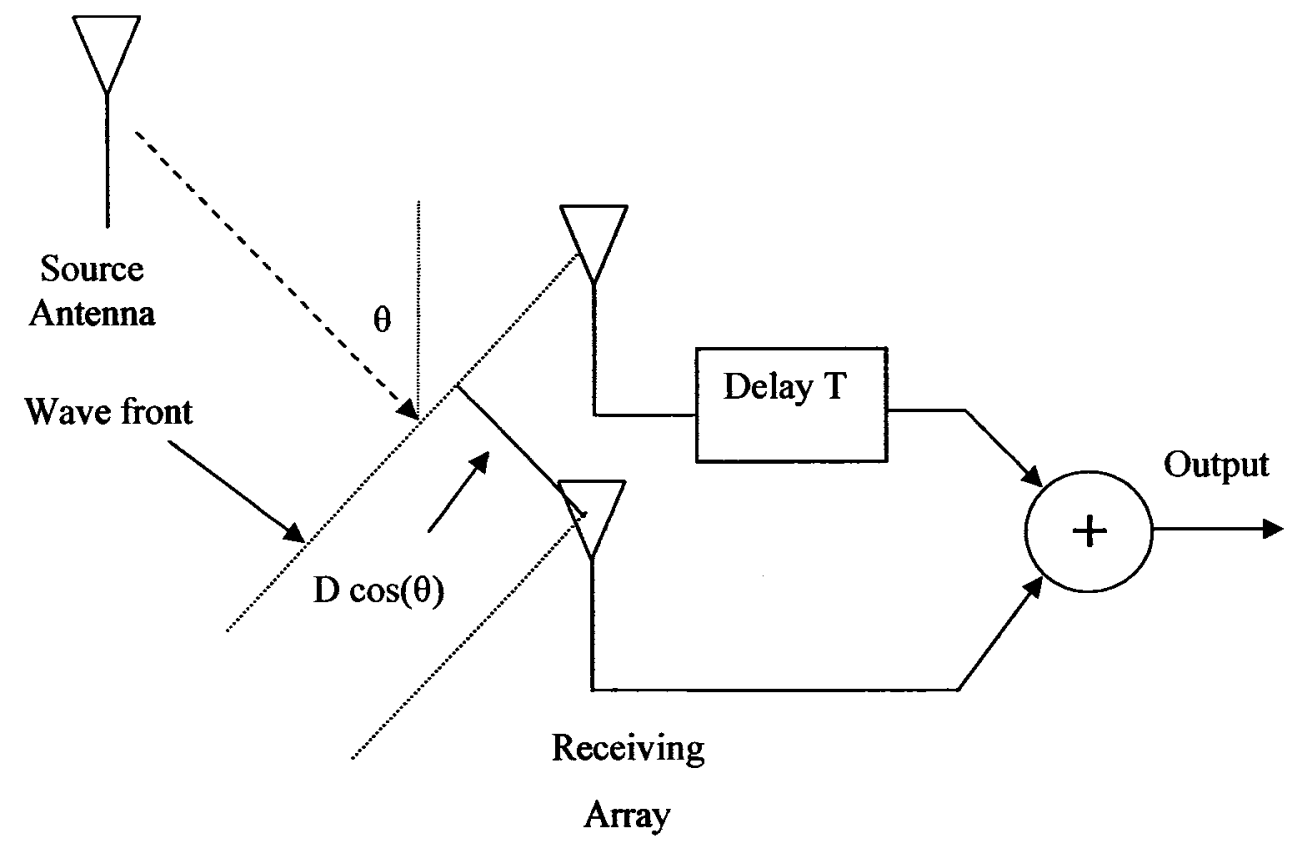

Figure A.3. Delay and sum beamformer.

(using delay-and-sum) the interference signal and then subtracting it from the array output. This method is cumbersome as the number of interference sources increases.

Another approach by Godara [95] and further developed by [96] [97] [98], steers a unity response towards the desired user and nulls undesired $n_{U}$ users by estimating the weight vector $\mathbf{w}$ such that

$$
\begin{aligned}
& \mathbf{w}^{H} \mathbf{s}_{0}=1 \\
& \mathbf{w}^{H} \mathbf{s}_{k}=0 \quad k=1,2, \cdots, n_{U}
\end{aligned}
$$

which can be written as

$$
\mathbf{w}^{H} \mathbf{A}=\mathbf{c}^{T}
$$


where

$$
\mathbf{A}=\left[\mathbf{s}_{0}, \mathbf{s}_{1}, \cdots, \mathbf{s}_{n_{u}}\right]
$$

and

$$
\mathbf{c}=[1,0, \cdots, 0]^{T}
$$

By making the number of BS antennas $n_{T}=n_{U^{+}}+1, \mathrm{~A}$ will become a square matrix and if the vectors $s_{i}$ are linearly independent then $w$ should be:

$$
\mathbf{w}=\left(\mathbf{c}^{T} \mathbf{A}^{-1}\right)^{H}
$$

So $\mathbf{w}$ is the first row of $\mathbf{A}^{-1}$ which gives unity response to the desired user and nulls the unwanted users. If $\mathbf{A}$ is not square then a pseudo-inverse of $\mathbf{A}$ can be used instead

$$
\mathbf{w}=\left(\mathbf{c}^{T} \mathbf{A}^{H}\left(\mathbf{A A}^{H}\right)^{-1}\right)^{H}
$$

The problem of this beamformer is that it enhances the noise on the received signal and it does not minimize the uncorrelated noise (as in the delay-and-sum case).

\section{A.2.3 Adaptive Beamformer}

This beamformer uses a pilot signal to estimate the filter weights (training process). It subtracts the array output from the pilot signal to produce an error signal used to optimize the filter weights by minimizing the MSE (between the pilot signal and the array output) as follows [99][100]

$$
\begin{aligned}
& e(n)=d(n)-\mathbf{w}^{H} \mathbf{u}(n) \\
& M S E=E\left\{e(n)^{2}\right\}=E\left\{|d(n)|^{2}-\mathbf{w}^{H} \mathbf{R} \mathbf{w}-2 \mathbf{w}^{H} \mathbf{p}\right\} \\
& \mathbf{p}=E\{\mathbf{u}(n) d(n)\}
\end{aligned}
$$

The solution of this minimization problem is the well known Wiener-Hoff equation 


$$
\mathbf{w}_{\text {opt }}=\mathbf{R}^{-1} \mathbf{p}
$$

The main disadvantage of this "one shot" solution is the complexity when the dimension of $\mathbf{R}$ increases.

\section{A.3 Nishimura Comparison of Nullsteering versus Beam Steering Beamformers}

Nishimura et al. considered a downlink SDMA system where the basestation, of $n_{T}$ antennas, is transmitting to $n_{U}$ users of one antenna each [17]. The received signal by the $k^{\text {th }}$ user is:

$$
r_{k}=\mathbf{g}_{k}^{T} \sum_{i=1}^{n_{U}} w_{i} s_{i}+n_{k}
$$

where the channel vector seen by the $k^{\text {th }}$ user is

$$
\mathbf{g}_{k}=\left[g_{1} g_{2} \cdots g_{n_{T}}\right]^{T}
$$

and the transmit filter is

$$
\mathbf{w}_{i}=\left[w_{1} w_{2} \cdots w_{n_{T}}\right]^{r}
$$

and $s_{i}$ is the signal for the $i^{t h}$ user, and finally $n_{k}$ is the noise.

Equation (A.16) can be written as follows to represent the signal vector received by all users

$$
\mathbf{r}=\mathbf{G W} \mathbf{s}+\mathbf{n}
$$

where

$$
\begin{aligned}
& \mathbf{r}=\left[\begin{array}{lll}
r_{1} & r_{2} \cdots r_{n_{U}}
\end{array}\right]^{T} \\
& \mathbf{G}=\left[\begin{array}{ll}
\mathbf{g}_{1} & \mathbf{g}_{2} \cdots \mathbf{g}_{n_{U}}
\end{array}\right]^{T} \\
& \mathbf{W}=\left[\begin{array}{lll}
\mathbf{w}_{1} & \mathbf{w}_{2} \cdots \mathbf{w}_{n_{U}}
\end{array}\right]
\end{aligned}
$$




$$
\begin{aligned}
& \mathbf{s}=\left[s_{1} s_{2} \cdots s_{n_{U}}\right]^{T} \\
& \mathbf{n}=\left[n_{1} n_{2} \cdots n_{n_{U}}\right]^{T}
\end{aligned}
$$

\section{A.3.1 Transmit MRC}

For transmit $\mathrm{MRC} \mathbf{W}=\mathbf{G}^{H}$ which makes the effective signal model be:

$$
\mathbf{r}=\mathbf{G G}^{H} \mathbf{s}+\mathbf{n}
$$

If the channel vectors of the users are uncorrelated then $\mathbf{G G}^{H}$ is almost diagonal and each user obtains his signal corrupted only by noise (interference free ).

\section{A.3.2 Transmit Null-steering}

For null steering, W should be transmit zeroforcing:

$$
\mathbf{W}=\mathbf{G}^{H}\left(\mathbf{G G}^{H}\right)^{-1}
$$

In case each user have $n_{R}$ antennas, the signal received by the $k t h$ user is:

$$
\mathbf{r}_{k}=\mathbf{G}_{k}\left[\mathbf{w}_{k} \mathbf{s}_{k}+\sum_{i=1, i \neq k}^{n_{U}} \mathbf{w}_{i} \mathbf{s}_{i}+\mathbf{n}\right]
$$

and by employing receive MRC then

$$
\begin{aligned}
r_{k} & =\left(\mathbf{G}_{k} \mathbf{w}_{k}\right)^{H} \mathbf{G}_{k} \mathbf{w}_{k} \mathbf{s}_{k}+\sum_{i=1, i \neq k}^{n_{U}} \mathbf{w}_{i} \mathbf{s}_{i}+\mathbf{n} \\
& =\mathbf{w}_{k} \mathbf{B}_{k} \mathbf{w}_{k} s_{k}+\text { impairment }
\end{aligned}
$$

where:

$$
\mathbf{B}_{k}=\mathbf{G}_{k}^{H} \mathbf{G}_{k}
$$


is a non-negative definite Hermitian matrix. For maximum SNR, the transmitted signal should be rotated along the direction of the eigenvector, $v_{k}^{\mathbf{B}_{k}, \max }$, that corresponds to the maximum eigenvalue of $\mathbf{B}_{k}$. By singular value decomposition,

$$
\mathbf{B}_{k}=\mathbf{U}^{\mathbf{B}_{k}} \mathbf{S}^{\mathbf{B}_{k}}\left(\mathbf{V}^{\mathbf{B}_{k}}\right)^{H}
$$

then,

$$
\mathbf{w}_{k}=v_{k}^{\mathbf{B}, \max }
$$

To null the interference caused by user $m$ on user $k$, the following condition must apply;

$$
\mathbf{w}_{m}^{H} \mathbf{B}_{k} \mathbf{w}_{m}=0
$$

This condition is satisfied when

$$
\mathbf{w}_{m}=v_{m}^{\mathbf{B}_{k}, \text { null }}
$$

is the eigenvector corresponding to a null eigenvalue.

To suppress the interference of user $k$ on all other users:

- constraint 1:

$$
\mathbf{w}_{m}^{H} \sum_{k=1, k \neq m}^{n_{U}} \mathbf{B}_{k} \mathbf{w}_{m}=0
$$

- constraint 2:

maximize the beam transmitted to the desired user:

$$
\max \left(\mathbf{w}_{m}^{H} \mathbf{B}_{m} \mathbf{w}_{m}\right)
$$

Generally speaking, the weight vector $\mathbf{w}_{m}$ is a linear combination of $N$ null eigenvectors as follows:

$$
\mathbf{w}_{m}=\sum_{n=1}^{N} c_{n}^{m} v_{n}^{\hat{\mathbf{B}}_{m}}
$$


which can be written as:

$$
\mathbf{w}_{m}=\widehat{\mathbf{E}}_{m} \mathbf{c}_{m}
$$

where

$$
\begin{aligned}
& \widehat{\mathbf{E}}_{m}=\left[\begin{array}{lll}
v_{1}^{\hat{\mathbf{B}}_{m}} & v_{2}^{\hat{\mathbf{B}}_{m}} \cdots v_{N}^{\hat{\mathbf{B}}_{m}}
\end{array}\right] \\
& \mathbf{c}_{m}=\left[\begin{array}{lll}
c_{1}^{m} & c_{2}^{m} \cdots c_{N}^{m}
\end{array}\right]^{T} \\
& \widehat{\mathbf{B}}_{m}=\sum_{k=1, k \neq m}^{n_{y}} \mathbf{B}_{k}
\end{aligned}
$$

The $c_{i}^{m}$ coefficients can be found from constraint 2 as follows;

$$
\mathbf{w}_{m}^{H} \mathbf{B}_{m} \mathbf{w}_{m}=\left(\widehat{\mathbf{E}}_{m} \mathbf{c}_{m}\right)^{H} \mathbf{B}_{m} \widehat{\mathbf{E}}_{m} \mathbf{c}_{m}=\mathbf{c}_{m}^{H} \widehat{\mathbf{E}}_{m}^{H} \mathbf{B}_{m} \widehat{\mathbf{E}}_{m} \mathbf{c}_{m}=\mathbf{c}_{m}^{H} \mathbf{F}_{m} \mathbf{c}_{m}
$$

so to maximize the above, the vector $c_{m}$ should be the eigenvector that corresponds to the maximum eigenvalue of $\mathbf{F}_{m}$ i.e

$$
\mathbf{c}_{m}=v_{\max }^{\mathbf{F}_{m}}
$$

Finally optimum transmit weight vector that maximizes the signal towards the desired user and nullify the undesired users is

$$
\mathbf{w}_{m}=\widehat{\mathbf{E}}_{m} \mathbf{c}_{m}=\left[\begin{array}{lll}
v_{1}^{\hat{\mathbf{B}}_{m}} & v_{2}^{\hat{\mathbf{B}}_{m}} \cdots v_{N}^{\hat{\mathbf{B}}_{m}}
\end{array}\right] v_{\max }^{\mathbf{F}_{m}}
$$

Simulations show that the nullsteering scheme outperforms the MRC scheme because of higher diversity gain. This scheme gets much more complicated with the increase of the number of users.

\section{A.4 Rim Downlink Nullsteering Technique}

Rim et al. proposed the following downlink nullsteering technique [19]. The input-output relationship can be written as: 


$$
\mathbf{r}_{k}=\mathbf{G}_{k} \mathbf{t}+\mathbf{n}_{k}
$$

where, for the $k^{\text {th }}$ user, $\mathbf{G}_{k}$ is channel matrix, $\mathbf{r}_{k}$ is the received signal vector, $\mathbf{n}_{k}$ is the received noise and $\mathbf{t}$ is

$$
\begin{aligned}
& \mathbf{t}=\sum_{\mathbf{i}=\mathbf{1}}^{K} \mathbf{W}_{i} \mathbf{x}_{i} \\
& \mathbf{W}_{k}=\mathbf{V}_{k} \mathbf{E}_{N}
\end{aligned}
$$

is the $n_{T} \times n_{R}$ beamforming matrix for the $k^{t h}$ mobile user, $\mathbf{E}_{N}$ is $n_{T} \times n_{R}$ with 1's on the main diagonal and 0 's otherwise.

The matrix $\mathbf{V}$ is obtained from the singular value decomposition of the channel matrix $\mathbf{G}$ :

$$
\mathbf{G}=\mathbf{U S V}^{H}
$$

To eliminate the interference caused by the $k^{\text {th }}$ user to all other users, $\mathbf{G}_{k}$ is chosen orthogonal to the matrix

$$
\mathbf{G}_{\bar{k}}=\left[\mathbf{G}_{1}^{H} \cdots \mathbf{G}_{k-1}^{H} \mathbf{G}_{k+1}^{H} \cdots \mathbf{G}_{K}^{H}\right]^{H}
$$

To make

$$
\mathbf{G}_{\bar{k}} \mathbf{W}_{k}=\mathbf{0}
$$

$\mathbf{W}_{k}$ is chosen to be;

$$
\mathbf{W}_{k}=\mathbf{V}_{k} \mathbf{E}_{N}-\mathbf{G}_{\bar{k}}^{H}\left(\mathbf{G}_{\bar{k}} \mathbf{G}_{\bar{k}}^{H}\right)^{-1} \mathbf{G}_{\bar{k}} \mathbf{V}_{k} \mathbf{E}_{N}
$$

then

$$
\mathbf{G}_{\vec{k}} \mathbf{W}_{k}=\mathbf{G}_{\bar{k}} \mathbf{V}_{k} \mathbf{E}_{N}-\mathbf{G}_{\bar{k}} \mathbf{G}_{\bar{k}}^{H}\left(\mathbf{G}_{\bar{k}} \mathbf{G}_{\bar{k}}^{H}\right)^{-1} \mathbf{G}_{\bar{k}} \mathbf{V}_{k} \mathbf{E}_{N}=0
$$

This fulfills the above constraint. 


\section{References}

[1] T. S. Rappaport, Wireless Communications - Principles and Practice. Prentice Hall, 1996.

[2] IEEE 802.16e committee, http://ieee802.org/16/tge.

[3] G. J. Foschini, "Layered space-time architecture for wireless communication in fading environments when using multi-element antennas," Bell Labs Techn. J., pp. 41-59, Autumn 1996.

[4] E. Telatar, "Capacity of multi-antenna Gaussian channels," European Trans. on Telecomm, vol. 10, no. 6, pp. 585-596, Nov. 1999.

[5] N. Seshadri and J. Winters, "Two signaling schemes for improving the error performance of frequency-division-duplex (FDD) transmission systems using transmitter antenna diversity," Int. J. Wireless Information Networks, vol. 1, pp. 49-60, Jan. 1994.

[6] S. Alamouti." A simple transmit diversity technique for wireless communications," IEEE Journal of Selected Areas in Communications., vol. 16, no. 8, pp. 14511458, Oct 1998.

[7] V. Tarokh, N. Seshadri and A. R. Calderbank, "Space-time codes for high data rate wireless communication: Performance criterion and code construction," IEEE Transactions on Information Theory, vol. 44, no. 2, pp. 744-765, Mar 1998.

[8] V. Tarokh, H. Jafarkhani and A. R. Calderbank, "Space-time block codes from orthogonal designs," IEEE Transactions on Information Theory, vol. 45, no. 5, pp. 1456-1467, July 1999.

[9] C. Papadias and G. Foschini, "On the capacity of certain space-time coding schemes," in Proc. EURASIP J. Appl. Signal, May 2002, vol. 5, pp. 447-458.

[10] T. lo, "Maximal ratio transmission," IEEE Transactions on Communications, vol. 47, no. 10, pp. 1458-1461, October 1999.

[11] G. J. Foschini, G. D. Golden, R. A. Valenzulea, and P.W. Wolniansky, "Simplified processing for high spectral efficiency wireless communication employing multiantenna arrays," IEEE Journal of Selected Areas in Communications, vol. 17, no. 11, pp. 1841-1852, 1999.

[12] G. D. Golden, G. J. Foschini, R. A. Valenzulela, and P. W. Wolniansky, "Detection algorithm and initial laboratory results using the V-BLAST space-time communication architecture," Electronic Letters, vol. 35, no. 1, pp. 14-15, 1999.

[13] D. Gerlach, "Adaptive transmitting antenna arrays at the base station in mobile radio networks. Ph.D dissertation," Depart. Of Elect. Eng., Stanford University, 1995. 
[14] B. Ottersten, "Array processing for wireless communications," in Proc. Eighth IEEE Signal Processing Workshop on Statistical Signal and Array Processing, June 1996, pp. 466-473.

[15] R. Roy, "Spatial division multiple access technology and its application to wireless communication systems," in Proc. IEEE Vehicular Technology Conference, Phoenix, AZ, May 1997vol. 2, pp. 730-734,

[16] P. Vandenameele, Space Division Multiple Access for Wireless Local Area Networks. Norwell, MA, USA: Kluwer, 2001.

[17] T. Nishimura, T. Ohgane, Y. Ogawa, Y. Doi, and J. Kitakado, "Downlink beamforming performance for an SDMA terminal with joint detection," in Proc. IEEE Vehicular Technology Conference, Oct. 2001, vol. 3, pp. 1538-1542.

[18] T. Nishimura, Y. Takatori, T. Ohgane, Y. Ogawa, and K. Cho, "Transmit nullforming for a MIMO/SDMA downlink with receive antenna selection," in Proc. IEEE Vehicular Technology Conference, Sept. 2002, vol. 1, pp. 190-194.

[19] M. Rim; "Multi-user downlink beamforming with multiple transmit and receive antennas", Electronics Letters, vol. 38, pp. 1725-1726, Dec. 2002.

[20] Lai-U. Choi, R. D. Murch, “A transmit preprocessing technique for multiuser MIMO systems using a decomposition approach," IEEE Transactions on Wireless Communications, vol. 3, no. 1, pp. 20-24, Jan. 2004.

[21] Q. H. Spencer, A. L. Swindlehurst, M. Haardt, "Zero-forcing methods for downlink spatial multiplexing in multiuser MIMO channels" IEEE Transactions on Signal Processing, vol. 52 ,no. 2 ,pp. 461-471, Feb. 2004.

[22] R. Van Nee, R. Prasad, OFDM for Wireless Multimedia Communications. London: Artech House, 2000.

[23] H. Bolcskei, D. Gesbert and A. Paulraj, "On the capacity of OFDM-based spatial multiplexing systems,". IEEE Transactions on Communications, vol. 50, pp. 225 234, February 2002.

[24] L. Schumacher, L. Berger, J. Moreno, "Recent advances in propagation characterization and multiple antenna processing in the 3GPP framework," in Proc of XXVIIth URSI General Assembly 2002, Maastricht, The Netherlands, Aug. 2002.

[25] A. F. Molisch, "MIMO Systems with Antenna Selection: An Overview," in Proc. Of IEEE Radio \& Wireless Conference (RAWCON), Aug 2003, pp. 167-170.

[26] A. F. Molisch, M. Z. Win and J. H. Winters, "Capacity of MIMO systems with antenna selection," in Proc. Of IEEE International Conference on Communications, vol. 2, pp. $570-574$, June 2001. 
[27] D. Gesbert, H. Bolcskei, D. A. Gore and A. J. Paulraj, "Outdoor MIMO wireless channels: models and performance prediction," IEEE Transactions on Communications, vol. 50, pp. 1926-1934, Dec 2002.

[28] D. S. Shiu, G. J. Foschini, M. J Gans, and J.M. Khan, "Fading correlation and its effect on the capacity of multi-element antenna systems," IEEE Transactions on Communications, vol. 48, pp. 502-513, March 2000.

[29] H. Sampath et al, "A fourth-generation MIMO-OFDM broadband wireless system: design, performance, and field trial results," IEEE Comm. mag., pp. 143-149, Sept. 2002.

[30] H. Al-Raweshidy, S. Komaki, Radio over fiber technologies for mobile communications networks. Norwood, MA, USA: Artech House, 1st edition, March 2002.

[31] J. S. Wu, J. Wu, and H. W. Tsao, "A radio-over-fiber network for microcellular system application," IEEE Transactions on Vehicular Technology, vol. 47,no. 1, pp. 84-94, Feb. 1998.

[32] J. Fan, C. L. Lu and L. G. Kazovsky, "Dynamic range requirements for microcellular personal communication systems using analog fiber-optic links," IEEE Transactions on Microwave Theory and Techniques, vol. 45, no. 8, pp. 1390-1397, Aug. 1997.

[33] I. Haroun, G. Chan, R. Hafez, L. Bouchard, and L. Boucher, "Feasibility study of radio over optical transmission systems for increased system efficiency", in Proc. of IEEE International Symposium on Electromagnetic Compatibility, Istanbul, Turkey, May 2003.

[34] G. J. Foschini and M. J. Gans, "On limits of wireless communications in a fading environment when using multiple antennas," Wireless Personal Communications: Kluwer Academic Press, no. 6, pp. 311-335, 1998.

[35] V. Erceg et. $A l$, "An empirically based path loss model for wireless channels in suburban environments," IEEE Journal of Selected Areas in Communications, vol. 17, no. 7, pp. 1205-1211, July 1999.

[36] V. Erceg et. Al, "Channel Models for Fixed Wireless Applications", Tech. Rep. IEEE 802.16 Broadband Wireless Access Working Group", IEEE 802.16.3c01/29r4, 17-07-2001.

[37] W.C. Jakes, D.O. Reudink, "Comparison of mobile radio transmission at UHF and X-band," IEEE Transactions on Vehicular Technology, vol. 16, pp. 10-13, Oct. 1967.

[38] L. J. Greenstein, V. Erceg, Y.S. Yeh, and M.V. Clark, "A new path-gain/delayspread propagation model for digital cellular channels," IEEE Transactions on Vehicular Technology, vol. 46, no. 2, May 1997. 
[39] A. Leon-Garcia, Probability and Random Processes for Electrical Engineering. Addison-Wesley Publishing Company, May 1994.

[40] G. L. Stuber, Principles of Mobile Communication. Norwell, USA: Kluwer Academic Publishers, 2001.

[41] D. Tse and P. Viswanath, Fundamentals of Wireless Communication. UK: Cambridge University Press, May 2005.

[42] C. N. Chuah, D. Tse, J. M. Kahn, and R. A. Valenzuela,"Capacity scaling in MIMO wireless systems under correlated fading," IEEE Transaction on Information Theory, vol. 48, pp. 637-650, Mar. 2002.

[43] Lucent technologies, "Further link level results for HSDPA using multiple antennas," Tech. rep. 3GPP TSG RI-00-1386, Nov. 2000.

[44] B. Vucetic and J. Yuan, Space-Time Coding. John Wiley, Chichester, U.K: May 2003.

[45] L. Schumacher, K. I. Pedersen and P. E. Mogensen, "From antenna spacings to theoretical capacities - guidelines for simulating MIMO systems," in Proc. of $13^{\text {th }}$ IEEE International Symposium on Personal Indoor Mobile and Radio Communications, Sept., 2002.

[46] J. Kermoal, L. Schumacher, K. Pedersen, P. Mogensen, and F. Frederiksen, "A stochastic MIMO radio channel model with experimental validation," IEEE Journal on Selected Areas in Communications, vol. 20, no. 6, pp. 1211-1226, Aug. 2002.

[47] A. Hottinen, O. Tirkkonen, R. Wichman, Multi-antenna transceiver techniques for $3 G$ and beyond. J. Wiley, UK, 2003.

[48] J. Salz, J. H. Winters, "Effect of fading correlation on adaptive arrays in digital mobile radio," IEEE Transactions on Vehicular Technology, vol. 43, pp. 1049 1057, Nov. 1994.

[49] K. I. Pedersen, P. E. Mogensen, and B. H. Fleury, "Spatial channel characteristics in outdoor performance," in Proc. of IEEE Vehicular Technology Conference, Ottawa, Canada, vol. 2, pp. 719-723, 1998.

[50] F. Adachi, M. Feeny, A. Williamson, and J. Parsons, "Cross-correlation between the envelopes of $900 \mathrm{MHz}$ signals received at a mobile radio base station site," in Proc. Of Inst. Elect. Eng., Pt. F., vol. 133, pp. 506-512, Oct. 1986.

[51] E. Biglieri, D. Divsalar, P. Mclane and M. Simon, Introduction to Trellis-Coded Modulation with Applications. Macmillan, New York, NY, 1991.

[52] R. Wesel and J. Cioffi, "Fundamentals of coding for broadcast OFDM," in Proc. of Asilomar Conf. on Signals, Systems and Computers, vol. 1, pp. 2-6, Pacific Grove, CA, Oct. 1995. 
[53] J.G. Proakis, and D.G. Manolakis, Digital signal processing: principles, algorithms and applications. Macmillan, 1996.

[54] E.Brigham, The fast Fourier Transform. Prentice Hall, Englewood Cliffs, NJ, 1974.

[55] R. Gray, "Toeplitz and Circulant Matrices: A Review," Available online at http://www-ee.stanford.edu/ gray/toeplitz.pdf.

[56] Z. Yang, H. Fang and C. Pan, "ACE with frame interleaving scheme to reduce peak-to-average power ratio in OFDM systems,", IEEE Transactions on Broadcasting, vol. 51, issue 4, pp. 571-575, Dec. 2005.

[57] H. Ryu, J. Lee and J. Park, "Dummy sequence insertion (DSI) for PAPR reduction in the OFDM communication system," IEEE Transactions on Consumer Electronics, vol. 50, issue 1, pp. 89-94, Feb 2004.

[58] P. Boonsrimuang, K. Mori, T. Paungma, and H. Kobayashi, "PAPR Reduction Method for OFDM Signal by Using Dummy Sub-carriers, "1st International Symposium on Wireless Pervasive Computing, pp. 1-5, Jan. 2006.

[59] F. Donmez, U. Aygolu, "A trellis based PAPR reduction method for OFDM," in Proc. of IEEE Signal Processing and Communications Applications, pp. 57 - 60, April 2004

[60] Nguyen Thanh Hieu, Sang-Woo Kiom and Heung-Gyoon Ryu, "PAPR reduction of the low complexity phase weighting method in OFDM communication system," Transactions on Consumer Electronics, vol. 51, issue 3, pp. 776-782, Aug. 2005

[61] Kwang Don Choe, Si Chul Kim and Park S.K., "Pre-scrambling method for PAPR reduction in OFDM communication systems," Transactions on Consumer Electronics, vol. 50, Issue 4, pp. 1044 - 1048, Nov. 2004.

[62] C. E. Shannon, "A mathematical theory of communication," Bell System Technical Journal, vol. 27, pp. 379-423 and 623-656, July and October, 1948.

[63] A. Paulraj, R. Nabar, D. Gore, Introduction to space-time wireless communications. U.K: Cambridge University Press, 2003.

[64] E. Biglieri, J . Proakis and S. Shamai, "Fading channels: Information-theoretic and communications aspects," IEEE Transaction of Information Theory, vol. 44, no. 6, pp. 2619-2692, Oct. 1998.

[65] L. Ozarow, S. Shamai and A. Wyner, "Information theoretic considerations for cellular mobile radio," IEEE Transactions on Vehicular Technology, vol. 43, no. 2, pp. 359-378, May 1994.

[66] G. H. Golub and C. F. Van Loan, Matrix Computations. Baltimore, MD: Johns Hopkins University Press, 1996. 
[67] C. Chuah, J. Kahn and D. Tse, "Capacity of multi-antenna array systems indoor wireless environment," in Proc. of IEEE Globecom, vol. 4, pp. 1894-1899, Sydney, Australia, Nov. 1998.

[68] T. Cover, J. Thomas, Elements of Information Theory. New York: J. Wiley, 1991.

[69] J. B. Andersen, "Antenna arrays in mobile communications: Gain, diversity, and channel capacity," IEEE Antennas Propagat. Mag., vol. 42, pp. 12-16, April 2000.

[70] M. Z. Win and J. H. Winters, "Analysis of hybrid selection/maximal-ratio combining of diversity branches with unequal SNR in Rayleigh fading," in Proc. of IEEE Vehicular Technology Conf., vol. 1, pp. 215-220, May 1999.

[71] A.F. Molisch, M.Z. Win, and J.H. Winters, "Reduced-complexity transmit/receive diversity systems," IEEE Transactions of Signal Processing, vol. 51, pp. 27292738, Nov. 2003.

[72] D. A. Gore, R. W. Heath and A. J. Paulraj, "Transmit selection in spatial multiplexing systems," IEEE Communication Letters, vol. 6, pp. 491-493, Nov. 2002.

[73] A.F. Molisch, M.Z. Win, and J.H. Winters, "Reduced-complexity transmit/receivediversity systems," in Proc. of IEEE Vehicular Technology Conference, pp. 1996$2000,2001$.

[74] L. Zheng, D.N.C. Tse, "Diversity and multiplexing: a Fundamental tradeoff in multiple antenna Channels", IEEE transactions on information theory, vol. 49, no. 5, pp. 1073-1096, May 2003.

[75] M. Godavarti, T. Marzetta and S. Shamai, "Capacity of a mobile multipleantenna wireless link with isotropically random Ricean fading," in Proc. of ICEE ISIT, vol. 323, Washington, DC, June 2001.

[76] P. Soma, D. S. Baum, V. Erceg, R. Krishnamoothy, and A. Paulraj, "Analysis and modeling of MIMO radio channel based on outdoor measurements conducted at 2.5GHz for fixed BWA applications," in Proc. of IEEE International Conference on Communications, vol.1, pp.272-276, May 2002.

[77] C. Yang-Seok, A. F. Molisch, M. Z. Win, J. H. Winters, "Fast algorithms for antenna selection in MIMO systems," in proc. of IEEE Vehicular Technology Conference, vol. 3, pp. 1733 - 1737,0ct. 2003.

[78] M. C. Tournier, et al., "Detailed design report on algorithms for MTMR system including classical approaches," IST-2001-38354, Strike project-D4.1, June 2003.

[79] O.J. Dunn and V.A. Clark, Applied statistics: analysis of variance and regression, John Wiley, 1974.

[80] K. Pearson, "Mathematical contributions to the theory of evolution. III. Regression, heredity and panmixia," Philos. Trans. Royal Soc. London Ser. A , 187, pp. 253-318, 1896. 
[81] G. Bao, J. Holtzman and D. Oses, "Fairness criteria for 1xEV-DV with more simulation results," Tech. Rep. 3GPP2 simulation Ad Hoc, QUALCOMM, Jan. 2001.

[82] B. Widrow and J. M. McCool, "A comparison of adaptive algorithms based on the methods of steepest decent and random search," IEEE Transactions of antennas and propagation, vol. 24, no. 5, pp. 615-636, Sept. 1976.

[83] A. Benveniste, M. Metivier, and P. Priouret, Adaptive algorithms and stochastic approximations. Springer-Verlag, 1990.

[84] S. Haykin, Adaptive filter theory. New Jersey: Prentice Hall, 2002.

[85] J. Treichler, C.R. Johnson, M.G. Larimore, Theory and design of adaptive filters. USA: Prentice Hall, 2001.

[86] B. Suard, G. Xu and T. Kailath, "Uplink channel capacity of space-divisionmutiple-access schemes," IEEE Transactions on Information Theory, vol. 44, no. 4, pp. 1468-1476, July 1998.

[87] O. Macchi, Adaptive processing: the least mean square approach with applications in transmission. Wiley, 1995.

[88] V. Solo, X. Kong, Adaptive signal processing algorithms: stability and performance. Prentice Hall, 1995.

[89] L. Godara, "Applications of antenna arrays to mobile communications, part I: performance improvement, feasibility, and system considerations," in Proc. of the IEEE, vol. 85, no. 7, pp. 1031-1060. July 1997.

[90] M. Mizuno and T Ohgane, "Application of adaptive array antennas to radio communications," Electronic Communication, vol. 77, pp. 48-59, Japan, 1994.

[91] M. Costa, "Writing on dirty paper," IEEE Transaction of Information Theory, vol. 29, no. 3, pp. 439-441, May 1983.

[92] L. Godara, "Applications of antenna arrays to mobile communications, part II: beam-forming and direction of arrival considerations," in Proc. of the IEEE, vol. 85, no. 8, pp. 1195-1244. July 1997.

[93] J. Fernandez, I. R. Corden, and M. Barrett, "Adaptive array algorithms for optimal combining in digital mobile communication systems," in Proc. Inst. Elect. Eng. Int. Conf. Antennas Propagation, Edinburgh, Scotland, pp. 983-986. 1993.

[94] V. C. Anderson, "DICANNE, a realizable adaptive process," IEEE J. Acoust. Soc. Amer, vol. 45, pp. 398-405, 1969.

[95] L. C. Godara and A. Cantoni, "Uniqueness and linear independence of steering vectors in array space," J. Acoust. Soc. Amer., vol. 70, pp. 467-475, 1981. 
[96] Y. Bresler, V. U. Reddy and T. Kailath, "Optimum beamforming for coherent signal and interferences," IEEE Transactions on Acoustics, Speech and Signal Processing, vol. 36, pp. 833-843, 1988.

[97] S. Choi, T. K. Sarkar, and S. S. Lee, "Design of two dimensional Tseng window and its application to antenna array for the detection of AM signal in the presence of strong jammers in mobile communications," Signal Process., vol. 34, pp. 297$310,1993$.

[98] I. Chiba, T. Takahashi, and Y. Karasawa, "Transmitting null beam forming with beam space adaptive array antennas," in Proc. of IEEE Vehicular Technology Conf., Stockholm, Sweden, 1994, pp. 1498-1502.

[99] B. Widrow, P. E. Mantey, L. J. Griffiths, and B. B. Goode, "Adaptive antenna systems," Proc. IEEE, vol. 55, pp. 2143-2158, 1967.

[100] B. Widrow, J. R. Glover, J. M. McCool, J. Kaunitz, C. S. Williams, R. H. Hearn, J.R. Zeidler, E. Dong, Jr., and R. C. Goodlin, "Adaptive noise canceling: Principles and applications," Proc. IEEE, vol. 63, pp. 1692-1716, 1975. 